\title{
Herleitung einer prozessorientierten Coaching-Forschungslandkarte und Bestimmung rahmensetzender Merkmale zur Untersuchung «bedeutsamer Momente» im Coaching
}

Zur Verortung der hier vorgelegten Studie wird in diesem Kapitel zunächst eine prozessorientierte Forschungslandkarte für Coaching skizziert. Darauf bezogen, wird das Forschungsdesiderat präzisiert, das in der Studie bearbeitet werden soll.

Angelehnt an ein Coaching-Evaluationsmodell, das den Anspruch erhebt, den aktuellen Stand der Forschung abzubilden, wird zu diesem Zweck zunächst die gegenwärtige Coaching-Forschung bilanziert. Nach einer Kritik am Prozessverständnis, das im vorgestellten Evaluationsmodell implizit enthalten ist und das stellvertretend für die Ausrichtung der aktuellen Coaching-Forschung steht, erfolgt dann ein Exkurs in ein Psychotherapie-Forschungsparadigma. Dieses in besonderer Weise das Verhältnis von Therapie- und Patienten-Prozess betonende Forschungsparadigma stellt die Grundlage dar für die Herleitung der oben genannten, prozessorientierten Forschungslandkarte für Coaching.

In der darauf folgenden, zweiten Darstellung des Forschungsstands werden Studien mit Bezug zu dieser Forschungslandkarte vorgestellt, die in der ersten Bilanz noch nicht berücksichtigt wurden. Damit wird deutlich, dass erste bedeutsame Studien im Sinne der skizzierten Forschungslandkarte bereits existieren. Untersuchungen zu «bedeutsamen Momenten», basierend auf der Analyse faktischer Coaching-Verläufe, existieren freilich noch keine. Abschließend werden in diesem Kapitel, in Anlehnung an ausgewählte Forschungsansätze der Psychotherapie-Forschung, erste methodologische, theoretische wie auch methodische Aspekte der hier vorgelegten Studie bestimmt. Alle diese Aspekte dienen ge-

(C) Der/die Autor(en) 2019

R. Wegener, Bedeutsame Momente im Coaching, https://doi.org/10.1007/978-3-658-25687-6_2 
wissermaßen als Grundlage für die weitere Ausarbeitung des Forschungsdesigns zur Analyse «bedeutsamer Momente» im Coaching (Kapitel 3).

\subsection{Erste Bilanzierung des Forschungsstands}

In diesem Abschnitt wird eine erste Bilanz zum aktuellen Stand der Coaching-Forschung hinsichtlich sowohl der Verbreitung als auch inhaltlicher Erkenntnisse gezogen.

\subsubsection{Etablierung von Coaching als Gegenstand der Wissen- schaft}

Die Verbreitung der Forschung zu Coaching wird anhand verschiedener Indikatoren verdeutlicht: Volumen wissenschaftlicher Beiträge und Übersichten zu Fachjournals, Lehrbüchern sowie Reviews und Metaanalysen.

\section{Menge produzierter wissenschaftlicher Beiträge}

Der Forschung kommt für die Professionalisierung von Coaching große Bedeutung zu. Für die Produktion wissenschaftlicher Beiträge gibt die Auswertung von Grant (2011) sowohl zur Menge als auch zur Qualität einen zusammenfassenden Überblick. So bilanziert Grant, dass seit dem Jahr 2000 bis zum 1. Januar 2011634 peer-reviewte und englisch verfasste Artikel zu Coaching erschienen sind (vgl. Abbildung 2.1). Davon enthält ein Drittel, exakt 231, empirische Daten. Nur vierzehn Artikel basieren auf randomisiert kontrollierten Interventionsstudien. ${ }^{3}$

3 Leider liegen gemäß Grant, wie er dem Autor in einem Austausch im Mai 2015 erklärt hat, keine weiter in die Gegenwart reichenden Auswertungen zum Stand der peer-reviewten Artikel vor. Aufgrund des hohen Aufwands ist keine weitere solche Recherche geplant. 


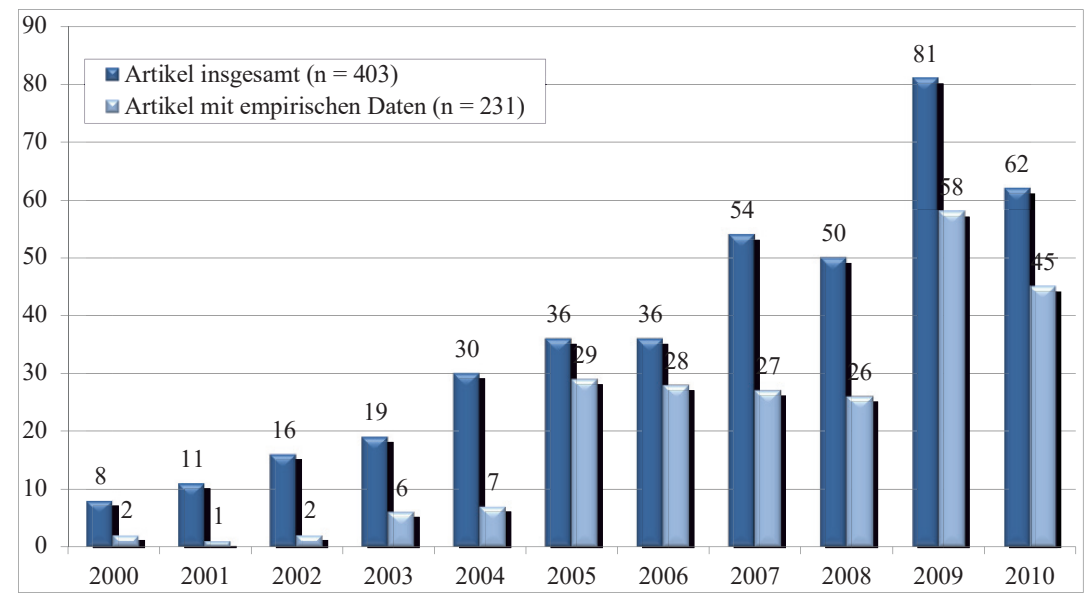

Abbildung 2.1 Wissenschaftliche Publikationen von 2000 bis 2010 (Quelle: Grant 2011, S. 2)

In Ergänzung zu dieser Auswertung hat eine Datenbankrecherche der Universität Kassel (Kotte et al. 2016, S. 6f.) zum Stichwort «Coaching» 44935 Treffer in peer-reviewten Journals ergeben (vgl. Tabelle 2.1). Für einen mit Grants Auswertung vergleichbaren Zeitraum von 2001 bis 2010 kommen die vier Autorinnen auf mehr als 30000 Beiträge, also beinahe fünfzigmal mehr als Grant. Sie haben aber im Unterschied zu Grant nicht geprüft, worin die Inhalte der identifizierten Beiträge genau bestehen. Entsprechend verweisen sie kritisch darauf, dass die große Zahl an Studien mit Vorsicht zu genießen sei, nicht zuletzt, weil «Coaching» als Containerbegriff in allen möglichen Zusammenhängen verwendet werde.

Tabelle 2.1 Datenbankrecherche zu «Coaching» (Quelle: Kotte et al. 2016, S. 6f.)

\begin{tabular}{lrc}
\hline Zeitraum & \multicolumn{1}{c}{$\begin{array}{l}\text { Anzahl der Treffer } \\
\text { «Titel: Coaching» }\end{array}$} & $\begin{array}{l}\text { Anzahl Treffer } \\
\text { «Titel: Executive Coaching» }\end{array}$ \\
\hline vor 1980 & 890 & 19 \\
\hline $1981-1990$ & 1865 & 24 \\
\hline $1991-1995$ & 1763 & 66 \\
\hline $1996-2000$ & 2561 & 184 \\
\hline $2001-2005$ & 5335 & 516 \\
\hline $2006-2010$ & 12848 & 1087 \\
\hline Summe & 19673 & 1291 \\
\hline
\end{tabular}


Interessant an dieser Darstellung ist der Befund, dass die Anzahl Treffer seit den 1980er Jahren exponentiell gewachsen ist. Dies spiegelt eine Entwicklung, die sich wohl auch in Zukunft fortsetzen wird. Der Begriff «Coaching» übt eine große Anziehungskraft aus, nicht nur in der Praxis, sondern offenbar mittlerweile auch im Zusammenhang mit wissenschaftlichen Publikationen. Wünschenswert wäre aber, dass viel häufiger deklariert wird, was in den Studien unter Coaching exakt verstanden wird, und dass diese Bestimmungen auch konsequent eingehalten werden (Künzli 2009, S. 8).

\section{Wissenschaftliche Journals}

Wie die Anzahl wissenschaftlicher Beiträge ist in den letzten Jahren auch die Anzahl wissenschaftlicher Journals mit «Coaching» als thematischem Schwerpunkt gestiegen. Bis auf eine Ausnahme handelt es sich bei allen in Tabelle 2.2 aufgeführten um Peer-Review-basierte Journals (Stand April 2016).

Tabelle 2.2 Peer-Review-basierte Journals mit thematischem Schwerpunkt «Coaching»

\begin{tabular}{ll}
\hline Journal (alphabetisch) & Website \\
\hline $\begin{array}{l}\text { Coaching: An International Journal of } \\
\text { Theory, Research and Practice }\end{array}$ & $\begin{array}{l}\text { http://www.tandfonline.com/toc/rcoa20/ } \\
\text { current }\end{array}$ \\
\hline $\begin{array}{l}\text { Coaching / Theorie \& Praxis (open } \\
\text { access) }\end{array}$ & $\begin{array}{l}\text { http://www.springer.com/psychology/ } \\
\text { beratung,+supervision,+coaching,+mediation/ } \\
\text { journal/40896 }\end{array}$ \\
\hline $\begin{array}{l}\text { International Coaching Psychology } \\
\text { Review }\end{array}$ & $\begin{array}{l}\text { http://shop.bps.org.uk/publications/ } \\
\text { publication-by-series/international-coaching- } \\
\text { psychology-review.html }\end{array}$ \\
\hline $\begin{array}{l}\text { International Journal of Coaching in } \\
\text { Organizations }\end{array}$ & http://www.ijco.info/ \\
\hline $\begin{array}{l}\text { International Journal of Evidence Based } \\
\text { Coaching and Mentoring (open access) }\end{array}$ & http://ijebcm.brookes.ac.uk/ \\
\hline $\begin{array}{l}\text { International Journal of Mentoring and } \\
\text { Coaching in Education }\end{array}$ & $\begin{array}{l}\text { http://www.emccouncil.org/eu/en/journal/ } \\
\text { journal_library }\end{array}$ \\
\hline $\begin{array}{l}\text { International Journal of Mentoring and } \\
\text { Coaching }\end{array}$ & http://www.emeraldinsight.com/toc/ijmce/5/1 \\
\hline $\begin{array}{l}\text { Organisationsberatung, Coaching, } \\
\text { Supervision (OSC)4 }\end{array}$ & http://link.springer.com/journal/11613 \\
\hline $\begin{array}{l}\text { The Coaching Psychologist } \\
\text { http://shop.bps.org.uk/publications/journals- } \\
\text { and-periodicals/the-coaching-psychologist- } \\
\text { vol-11-no-1-june-2015.html }\end{array}$ \\
\hline
\end{tabular}

$4 \quad$ Nicht «peer-reviewt». 


\begin{tabular}{ll}
\hline Journal (alphabetisch) & Website \\
\hline The Journal of Coaching Education & $\begin{array}{l}\text { http://www.qualitycoachingeducation.org/ } \\
\text { info-center/journal-of-coaching-education/ }\end{array}$ \\
\hline $\begin{array}{l}\text { International Journal of Sport Sciences } \\
\text { \& Coaching }\end{array}$ & $\mathrm{http://spo.sagepub.com/}$ \\
\hline
\end{tabular}

Beiträge zu Coaching werden auch in akademischen Journals veröffentlicht, die Coaching nicht zum exklusiven Gegenstand haben. Zu den Journals, in denen mehr als zehn Artikel zu Coaching publiziert wurden, gehören gemäß einer Recherche der Universität Kassel folgende vier (Kotte et al. 2016, S. 8):

- Consulting Psychology Journal: Practice and Research

- Journal of Management Development

- Personnel Psychology

- Industrial and Organizational Psychology: Perspectives on Science and Practice

Bis auf Personnel Psychology (a-Level) mit maximal hohem Ranking sind alle anderen b- und c-Level Journals. Entsprechend verweist auch Grant (2013) darauf, dass Coaching-Artikel mehrheitlich in b- und c-Level Journals veröffentlicht würden. Kotte et al. (2016, S. 8) erklären den Befund damit, dass die Coaching-Forschung noch ein junges Forschungsfeld sei. Bis Coaching «in die ganz großen Journals 〈aufsteigt»» (ebd.), muss sich das Forschungsfeld erst noch weiter etablieren. Damit verbunden wird auch der kritische Hinweis, dass es immer noch nur wenige quantitative RCT- und damit gut publizierbare Studien gibt.

\section{Reviews und Metaanalysen}

Die Zahl der seit 2000 veröffentlichten Reviews, in denen Forschungsergebnisse zu Coaching zusammengetragen werden und die Forschungslage bewertet wird, ist in den letzten Jahren stark gestiegen. Gleiches gilt für Metaanalysen, in denen Ergebnisse ausgewählter Studien quantifizierend zu Metadaten übergeführt werden (Kotte et al. 2016, S. 9).

Die folgende Übersicht (vgl. Tabelle 2.3) mit 41 Reviews und vier Metaanalysen ist das Ergebnis einer eigenen Literaturrecherche und einer Umfrage, die der Autor von 2015 bis 2017 durchgeführt hat. Dazu wurde zunächst eine Liste der Metaanalysen und Reviews zusammengestellt, die dem Autor im Verlauf seiner Recherchen begegnet sind. Diese Liste wurde den darin aufgeführten Autoren und Autorinnen im Mai 2015 zugestellt, verbunden mit der Bitte, sie um nicht auf- 
geführte Veröffentlichungen zu erweitern. ${ }^{5}$ Die Übersichtsliste wurde um die neu angegebenen Reviews und Metaanalysen - mit einem Sternchen (*) versehen ergänzt. Die mit zwei Sternchen (**) versehenen Beiträge wurden auf der Basis weiterer Recherchen des Autors bis 2017 aufgenommen.

Tabelle 2.3 Seit $2000 \mathrm{zu}$ «Coaching» veröffentlichte Reviews und Metaanalysen

\section{Reviews und Metaanalysen zu Coaching}

\section{Metaanalysen}

De Meuse, K. P., Dai, G., \& Lee, R. J. (2009). Evaluating the effectiveness of executive coaching: Beyond Roi? Coaching: An International Journal of Theory, Research and Practice, 2(2), 117-134.

*Jones, R. J., Woods, S. A., \& Guillaume, Y. R. F. (2015). The effectiveness of workplace coaching: A meta-analysis of learning and performance outcomes from coaching. Journal of Occupational and Organizational Psychology, 89(2), 249-277.

**Sonesh, S. C., Coultas, C. W., Lacerenza, C. N., Marlow, S. L., Benishek, L. E., $\&$ Salas, E. (2015). The power of coaching: a meta-analytic investigation. Coaching: An International Journal of Theory, Research and Practice, 8(2), 73-95.

Theeboom, T., Beersma, B., \& Vianen, A. E. M. v. (2013). Does coaching work? A meta-analysis on the effects of coaching on individual level outcomes in an organizational context. The Journal of Positive Psychology: Dedicated to furthering research and promoting good practice, 9(1), 1-18.

\section{Reviews}

Bachkirova, T., \& Kauffman, C. (2008). Many ways of knowing: How to make sense of different research perspectives in studies of coaching. Coaching: An International Journal of Theory, Research and Practice, 1(2), 107-113.

Bennett, J. L. (2006). An Agenda for Coaching-Related Research. A Challenge for Researchers. Consulting Psychology Journal: Practice and Research, 58(4), $240-249$.

Böning, U., \& Kegel, C. (2015). Ergebnisse der Coaching-Forschung. Aktuelle Studien-ausgewertet für die Coaching-Praxis. Wiesbaden: Springer.

*Bono, J. E., Purvanova, R. K., Towler, A. J., \& Peterson, D. (2009). A survey of executive coaching practices. Personnel Psychology, 62(2), 361-404.

Campone, F. (2011). Current Research on Coaching. In L. Wildflower \& D. Brennan (Eds.), The handbook of knowledge-based coaching: from theory to practice (pp. 329-340). San Francisco, CA: Jossey-Bass.

**Carey, W., Philippon, D., \& Cummings, C. (201). Coaching models for leadership development: an integrative review. Journal of Leadership Studies, 5(1), 51-69.

5 Dankenswerterweise haben folgende Forscher und Forscherinnen auf die Anfrage des Verfassers geantwortet: Tatiana Bachkirova, Erik de Haan, Anthony Grant, Siegfried Greif, Jennifer D. Irwin, Baek-Kyoo Joo, Carol Kauffman, Jim Knight, Jonathan Kyle Nelson, Gary P. Latham, William Morley, Jonathan Passmore, Tim Theeboom und James Smither. 
Cornett, J., \& Knight, J. (2009). Research on Coaching. In J. Knight (Ed.), Coaching: Approaches \& perspectives. Thousand Oaks, CA: Corwin Press.

Douglas, C. A., \& Morley, W. H. (2000). Executive coaching: an annotated bibliography. Greensboro, NC: Center for Creative Leadership.

Ellam-Dyson, V., \& Palmer, S. (2008). The challenges of researching executive coaching. The Coaching Psychologist, 4(2), 79-84.

Ely, K., Boyce, L. A., Nelson, J. K., Zaccaro, S. J., Hernez-Broome, G., \& Thymann, W. (2010). Evaluating leadership coaching: A review and integrated framework. The Leadership Quarterly, 21(4), 585-599.

Feldman, D. C., \& Lankau, M. J. (2005). Executive coaching: A Review and Agenda for Future Research. Journal of Management, 31(6), 829-848.

Fillery-Travis, A., \& Lane, D. (2007). Research: does coaching work? In S. Palmer \& A. Whybrow (Eds.), Handbook of Coaching Psychology (pp. 57-69). New York, NY: Routledge.

Garvey, R., Stokes, P., \& Megginson, D. (2009a). Researching Coaching and Mentoring. In R. Garvey, P. Stokes \& D. Megginson (Eds.), Coaching and Mentoring. Theory and Practice (pp. 28-52). London: Sage.

Grant, A. (2011). Workplace, Executive and Life Coaching: An Annotated Bibliography from the Behavioural Science and Business Literature ( $1^{\text {st }}$ Jan 2011). Sydney: Coaching Psychology Unit, University of Sydney, Australia.

*Grant, A. (2013). The Efficacy of Coaching. In J. Passmore, D. Peterson \& T. Freire (Eds.), The Wiley-Blackwell Handbook of the Psychology of Coaching and Mentoring (pp. 15-39). Chichester: Wiley-Blackwell.

Grant, A., Cavanagh, M., Parker, H., \& Passmore, J. (2010). The State of Play in Coaching Today: A Comprehensive Review of the Field. International Review of Industrial and Organizational Psychology, 25, 125-167.

Greif, S. (2007). Advances in research on coaching outcomes. International Coaching Psychology Review, 2(3), 222-249.

Greif, S. (2008). Stand der Evaluationsforschung. In S. Greif (Hrsg.), Coaching und ergebnisorientierte Selbstreflexion (S. 212-286). Göttingen: Hogrefe.

*Greif, S. (2013). Conducting organizational based evaluations of coaching and mentoring programs. In J. Passmore, D. B. Peterson \& T. Freire (Eds.), The WileyBlackwell Handbook of the Psychology of Coaching and Mentoring. (pp. 445-471). Chichester: Wiley-Blackwell.

Greif, S. (2016). Wie wirksam ist Coaching? Ein umfassendes Evaluationsmodell für Praxis und Forschung. In R. Wegener, M. Loebbert \& A. Fritze (Hrsg.), CoachingPraxisfelder. Forschung und Praxis im Dialog (2. Aufl., S. 161-182). Wiesbaden: Springer.

Greif, S., Schmidt, F., \& Thamm, A. (2012). Warum und wodurch Coaching wirkt. Ein Überblick zum Stand der Theoriebildung und Forschung über Wirkfaktoren. Organisationsberatung, Supervision, Coaching (OSC), 19(4), 375-390.

*Haan, E. de, \& Burger, Y. (2013). Choosing the right method. In E. de Haan \& Y. Burger (Eds.), Coaching with Colleagues. An Action Guide for One-to-One Learning ( $2^{\text {nd }}$ ed., pp. 122-140). Basingstoke: Palgrave Macmillan.

Haan, E. de, \& Duckworth, A. (2012). Signalling a new trend in executive coaching outcome research. International Coaching Psychology Review, 8(1), 6-19. 
Joo, B.-K. (2005). Executive Coaching: A Conceptual Framework From an Integrative Review of Practice and Research. Human Resource Development Review, 4(4), 462-488.

*Joo, B.-K., Sushko, J. S., \& McLean, G. N. (2012). Multiple faces of coaching: Manager-as-coach, executive coaching, and formal mentoring. Organizational Development Journal, 30(1), 19-38.

Kampa-Kokesch, S., \& Anderson, M.Z. (2001). Executive Coaching. A Comprehensive Review of the Literature. Psychology Journal: Practice and Research, 53(4), 205-228.

**Kotte, S., Hinn, D., Oellerich, K., \& Möller, H. (2016). Der Stand der Coachingforschung: Kernergebnisse der vorliegenden Metaanalysen. Organisationsberatung, Supervision, Coaching (OSC), 23(1), 5-23.

Künzli, H. (2009). Wirksamkeitsforschung im Führungskräfte-Coaching. Organisationsberatung, Supervision, Coaching (OSC), 16(1), 4-16.

Künzli, H., \& Seiger, C. (2011). Evidence-based Coaching und Wirksamkeit. Hamburg: Europäische Fernfachhochschule.

Latham, G. P. (2007). Theory and research on coaching practices. Australian Psychological Society, 42(4), 268-270.

Levenson, A. (2009). Measuring and Maximising the business impact of Executive Coaching. Consulting Psychology Journal: Practice and Research, 61(2), 103-121.

Linley, A. P. (2006). Coaching Research: Who? What? Where? When? Why? International Journal of Evidence Based Coaching and Mentoring, 4(2), 1-7.

**MacKie, D. (2007). Evaluating the effectiveness of executive coaching: Where we are now and where do we need to be. Australian Psychologist, 42(7), 310-318.

*Mazano, R. J., \& Simms, J. A. (2013). Research and Theory. In R. J. Mazano \& J. A. Simms (Eds.), Coaching Classroom Instruction (pp. 3-18). Bloomington, IN: Marzano Research Laboratory.

Möller, H., \& Kotte, S. (2011). Die Zukunft der Coachingforschung. Organisationsberatung, Supervision, Coaching (OSC), 18(4), 445-456.

Newnham-Kansas, C., Gorczynski, P., Morrow, D., \& Irwin, J. D. (2009). Annotated Bibliography of Life Coaching and Health Research. International Journal of Evidence Based Coaching and Mentoring, 7(1), 39-103.

Passmore, J., \& Fillery-Travis, A. (2011). A critical review of executive coaching research: a decade of progress and what's to come. Coaching: An international Journal of Theory, Research and Practice, 4(2), 70-88.

Passmore, J., \& Gibbes, C. (2007). The state of executive coaching research: What does the current literature tell us and what's next for coaching research? International Coaching Psychology, 2(2), 116-128.

*Passmore, J., \& Theeboom, T. (2015). Coaching Psychology: A journey of development in research. In L. E. van Zyl, A. Oodendal \& M. W. Stander (Eds.), Coaching Psychology: Meta-theoretical perspectives and applications in multi-cultural contexts. New York, NY: Springer.

**Sforzo, G. A., Kaye, M. P., Todorova, I., Harenberg, S., Costello, K., Cobus-Kuo, L., Faber, A., Frates, E., \& Moore, M. (2017). Compendium of the Health and Wellness Coaching Literature. American Journal of Lifestyle Medicine. doi $10.1177 / 1559827617708562$. 
Smither, J. W., \& Reilly, S. P. (2001). Coaching in Organizations: A Social Psychological Perspective. In M. London (Ed.), How People Evaluate Others in Organizations: Person Perception and Interpersonal Judgment in I/O Psychology (pp. 221-252). Mahwah, NJ.: Lawrence Erlbaum.

*Theeboom, T. (2016). The Current State of Research. In C. van Nieuwerburgh (Ed.), Coaching in Professional Contexts (pp. 187-198). London: Sage.

\section{Lehr- und Handbücher zu Coaching}

Inzwischen gibt es auch eine Fülle von Lehr- und Handbüchern zu Coaching. Ihr Inhalt basiert mehrheitlich auf wissenschaftlich-konzeptionellen Grundlagen (vgl. dazu auch Geißler 2016, S. 29). Die folgende Übersicht (Tabelle 2.4) erhebt nicht den Anspruch auf Vollständigkeit, will aber einen entsprechenden Überblick über einige Werke geben, die dem Autor auf dem Weg seiner Recherchen wiederholt begegnet sind.

Tabelle 2.4 Lehr- und Handbücher zu Coaching

\section{Lehr- und Handbücher zu Coaching}

Bachkirova, T., Spence, G., \& Drake, D. (Eds.) (2017). The SAGE Handbook of Coaching. London: Sage.

Cox, E., Bachkirova, T., \& Clutterbuck, D. (Eds.) (2014). The Complete Handbook of Coaching ( $2^{\text {nd }}$ ed.). London: Sage.

Garvey, R., Stokes, P., \& Megginson, D. (2009b). Coaching and Mentoring. Theory and Practice. London: Sage.

Greif, S. (2008). Coaching und ergebnisorientierte Selbstreflexion. Göttingen: Hogrefe.

Hudson, F. M. (1999). The Handbook of Coaching. A Comprehensive Resource Guide for Managers, Executives, Consultants, and Human Resource Professionals. San Francisco, CA: Jossey-Bass.

Law, H. (2013). The Psychology of Coaching, Mentoring and Learning ( $2^{\text {nd }}$ ed.). Chichester: Wiley-Blackwell.

Lippmann, E. (2013). Coaching: Angewandte Psychologie für die Beratungspraxis (3. Aufl.). Berlin: Springer.

Loebbert, M. (2015). Coaching Theorie. Eine Einführung. Wiesbaden: Springer.

Palmer, S., \& Whybrow, A. (Eds.) (2008). Handbook of coaching psychology: a guide for practitioners. London: Routledge.

Passmore, J., Peterson, D., \& Freire, T. (Eds.) (2013). The Wiley-Blackwell Handbook of the Psychology of Coaching and Mentoring. Chichester: Wiley-Blackwell.

Peltier, B. (2010). The psychology of executive coaching: theory and application ( $2^{\text {nd }}$ ed.). New York: Routledge. 
Rush, D. D., \& Shelden, M., \& Lisa, L. (2011). The early childhood coaching handbook. Maryland: Brookes.

Stober, D. R., \& Grant, A. M. (Eds.) (2006). Evidence based coaching handbook: putting best practices to work for your clients. Hoboken, NJ: Wiley.

Wildflower, L., \& Brennan, D. (Eds.) (2011). The handbook of knowledgebased coaching: from theory to practice. San-Francisco, CA: JosseyBass.

\section{Fazit}

Der Überblick verdeutlicht, dass wissenschaftliche Bemühungen im Zuge der aktuellen Professionalisierung von Coaching ein bereits ansehnliches Ausmaß erreicht haben. Neben Fachartikeln gibt es immer mehr Journals, Reviews, Metaanalysen sowie Lehr- und Handbücher. Das junge Forschungsfeld ist demnach, wie Kotte et al. (2015, S. 29) es formulieren, «auf dem Vormarsch». Entsprechend meinte jüngst auch Anthony Grant, ${ }^{6}$ ein renommierter Coaching-Forscher aus Australien, es werde immer schwieriger, den Überblick über die akademischen Veröffentlichungen zu Coaching zu behalten.

Interessant ist nun die Frage, was die Coaching-Forschung auf der inhaltlichen Ebene an Wissen und Erkenntnissen produziert hat.

\subsubsection{Forschungsstand zu Coaching, orientiert am Evaluationsmodell von Greif}

Ein Überblick über den Stand der Coaching-Forschung setzt voraus, dass man sich Gedanken macht, wie das entsprechende Forschungsfeld und damit verbundene Ergebnisse strukturiert werden können. Fehlt eine solche Grundlage, so droht Gefahr, sich in der Vielfalt vorhandener Forschungsresultate zu verlieren. Jede derartige Setzung hat allerdings auch zur Folge, dass gewisse Studien und Ergebnisse nicht berücksichtigt werden.

Ein möglicher Strukturierungszugang orientiert sich an den Standards der Evaluationsforschung. Sozialwissenschaftliche Evaluation bedeutet, Konzepte zu sozialen Interventionen - hier Coaching -, deren Umsetzung und Nutzen durch eine systematische Anwendung sozialwissenschaftlicher Methoden zu bewerten (Rossi \& Freeman 1993).

6 Vgl. seine Keynote an der Annual Conference in Leadership and Health Coaching, Boston, USA (13./14. Oktober 2017). 
Eine entsprechende Strukturierungsvariante, die in Reviews und Darstellungen der Coaching-Forschung bereits punktuell Anwendung findet (z. B. Ely et al. 2010; Greif 2008; Möller \& Kotte 2011), folgt der Unterscheidung von summativer und formativer Evaluation (vgl. Scriven 1980; Scriven 1996). Summative Evaluationen zielen auf die wissenschaftliche Bewertung finaler Ergebnisse von durchgeführten Interventionen ab. Formative Evaluationen verfolgen dagegen das Ziel, Aspekte zu erfassen, die im Zusammenhang mit der Qualität einer Intervention stehen.

An diese Unterscheidung schließt auch das 2008 veröffentlichte Strukturmodell der Coachingwirkungen von Greif (2008) an. Dieses Modell, das eine Vielzahl von Dimensionen und darin enthaltene Variablen umfasst, wurde zu einem umfassenden Coaching-Evaluationsmodell weiterentwickelt (Greif 2013; Greif 2016). Seinen Ursprung hat es in einer früheren Modellentwicklung, basierend auf Recherchen zum damals aktuellen Forschungsstand. Weiter erhebt es den Anspruch, den derzeitigen Stand der Fachliteratur zu erfassen (Greif 2016, S. 168). Inhaltlich umfasst es Ergebnisse neuerer Reviews (Grant et al. 2010; Theeboom, Beersma \& van Vianen 2013), Konstrukte des Mentoring-Evaluationsmodells nach Wanberg, Welsh und Hezlett (2011) sowie Messskalen von Ely und Zaccaro (2011) (vgl. Greif 2016, S. 168).

Das Modell und darauf bezogene Forschungsergebnisse werden aufgrund seiner weiten Verbreitung und internationalen Rezeption sowie des damit erhobenen Anspruchs, den aktuellen Stand der Fachliteratur abzubilden, im Sinne einer ersten Forschungsbilanzierung nun vorgestellt. Anschließend an die Würdigung werden die dem Modell zugrunde liegende Ausrichtung und insbesondere das darin enthaltene Prozessverständnis kritisch diskutiert. Die nun folgende Darstellung versteht sich deshalb als Hinführung zur gegenwärtig dominanten Ausrichtung der Coaching-Forschung und zweitens als Ausgangspunkt für ein Plädoyer zu deren deutlich prozessorientierterer Ausrichtung.

\section{Das Coaching-Evaluationsmodell von Greif}

Der Unterscheidung von formativer und summativer Evaluation folgend, teilt Greif (2016) in seinem Evaluationsmodell Coaching und die darauf bezogene Forschung in «Voraussetzungen», «Coaching-Prozess» sowie «kurz- und langfristige Coaching-Ergebnisse» ein (vgl. Abbildung 2.2). Die Dimensionen «Voraussetzungen» und «Ergebnisse» werden unterteilt in die Perspektiven «Coach», «Kunde» und «Organisation». Bei den «kurzfristigen Ergebnissen» werden «allgemeine» und «spezifische Variablen» unterschieden, beim «Coaching-Prozess» die Dimensionen «Coaching-Beziehung», «Verhalten der Coaches» sowie «Merkmale und Verhalten der Kunden». 

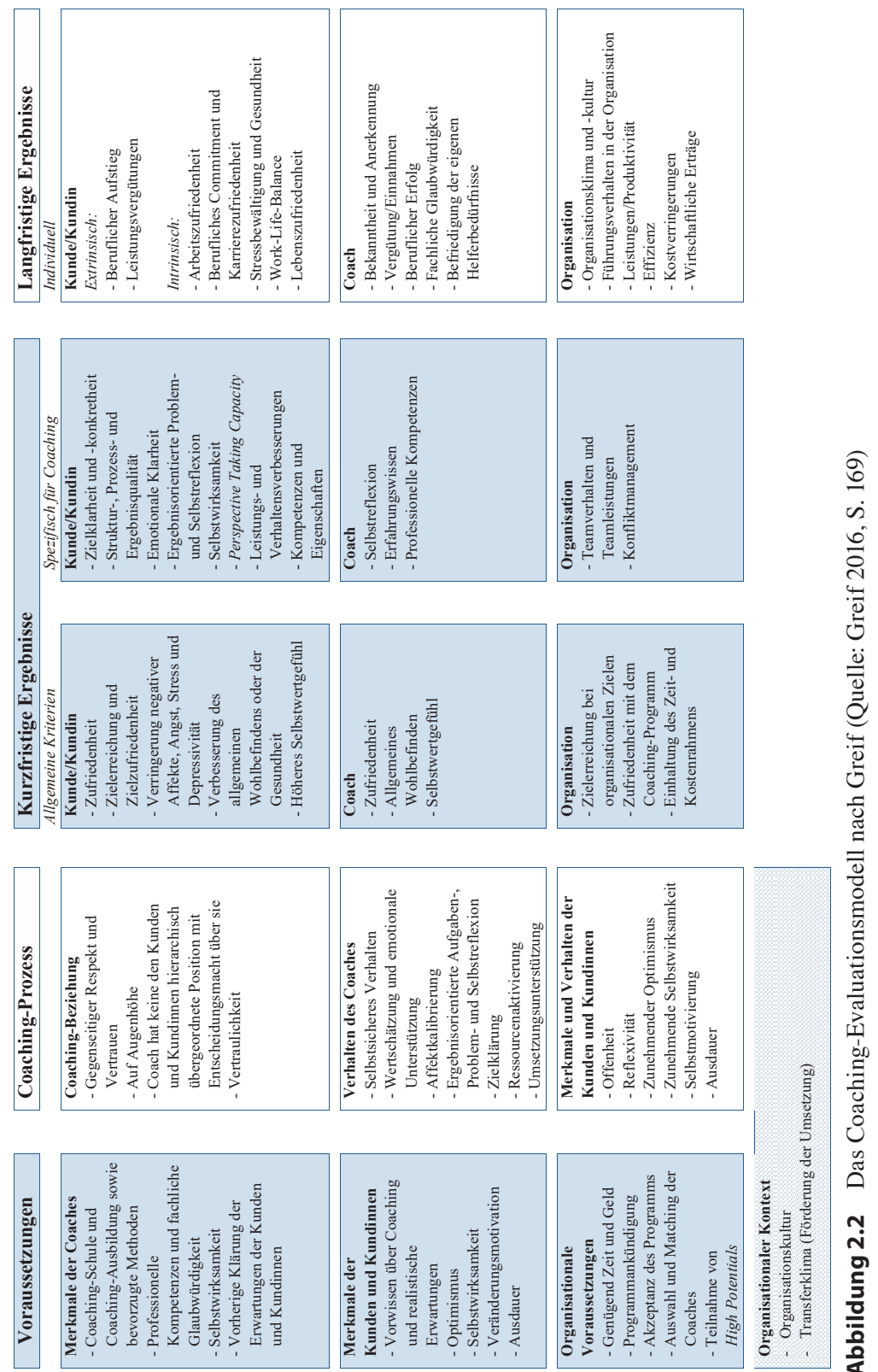
Greif argumentiert, dass innerhalb der von ihm berücksichtigten Dimensionen auf theoretischer Ebene weitere Variablen einbezogen werden könnten, er jedoch nur jene berücksichtige, für die auch empirische Untersuchungen vorliegen (Greif 2016).

\section{Voraussetzungen und organisationaler Kontext}

Bezogen auf die Dimension «Voraussetzungen», verschiedene Merkmale des «Coaches», des «Kunden» und der «Organisation» sowie hinsichtlich des «organisationalen Kontexts», beschreibt Greif (2016, S. 170f.) allgemeine Merkmale (vgl. Abbildung 2.2, erste «Spalte»), die aufgrund der Forschungsbefunde mit dem Erfolg von Coaching korrelieren.

Für den Coach ist auf der Ebene der «Voraussetzungen» zunächst die «Selbstwirksamkeitsüberzeugung» erfolgsrelevant, das heißt die subjektive Gewissheit, ein Ziel erreichen zu können. Von Belang sind zudem die vom Coach vertretene «Coaching-Richtung/Schule», die «absolvierte Coaching-Ausbildung», die «bevorzugten Methoden», die «professionellen Kompetenzen», «fachliche Glaubwürdigkeit» und die durch den Coach durchgeführte «Klärung der Kundenerwartungen».

Als erfolgsrelevante Voraussetzungen der Kundinnen und Kunden benennt Greif aufgrund der Forschungslage das «Vorwissen über und die Erwartungen an ein Coaching». Relevant sind weiter «Optimismus», «Selbstwirksamkeitsüberzeugung», «Veränderungsmotivation» und «Ausdauer» der Kundinnen und Kunden.

Als erfolgsrelevante Voraussetzungen auf organisationaler Ebene benennt Greif die Ressourcen «Zeit» und «Geld», die «Ankündigung» und die «Akzeptanz des Coaching-Programms», die «Auswahl des und das Matching mit einem Coach» sowie die «prioritäre Berücksichtigung von High Potentials in neu eingeführten Coaching-Programmen».

Bezogen auf den «organisationalen Kontext», sind gemäß Greif die «Organisationskultur» und das «Transferklima», sprich eine förderliche Haltung des Umfelds mit Blick auf die in einem Coaching bearbeiteten Inhalte, für den Coaching-Erfolg zentral.

\section{Prozess}

Als Dimensionen des «Coaching-Prozesses» (vgl. Abbildung 2.2, zweite Spalte) unterscheidet Greif zwischen «Coaching-Beziehung», «Coach-Verhalten», «Kundenmerkmalen und -verhalten» (ebd., S. 171 f.).

Als erfolgsrelevante Teilaspekte der «Coaching-Beziehung» werden gegenseitiger «Respekt» und «Vertrauen», eine «Beziehung auf Augenhöhe», «Abwesenheit eines Machtgefälles zwischen Coach und Kunde» (d.h. keine Entscheidungsmacht des Coaches) und «Vertraulichkeit» bestimmt. 
Bei der Dimension «Coach-Verhalten» beruft sich Greif auf die von ihm eingeführten, teils aus der Psychotherapie-Forschung übernommenen «Coaching-Wirkfaktoren» (Greif 2008). Der Einfluss dieser Faktoren auf die finalen Coaching-Ergebnisse haben Greif und sein Team in ratingbasierten Untersuchungen untersucht (Greif 2015; Greif et al. 2010; Greif \& Schubert 2014). Gemäß neuesten Darstellungen zählen dazu (Greif 2016, S. 169, 171):

- selbstsicheres Verhalten,

- Wertschätzung und emotionale Unterstützung,

- Affektkalibrierung,

- ergebnisorientierte Aufgaben-, Problem- und Selbstreflexion,

- Zielklärung,

- Ressourcenaktivierung,

- Umsetzungsunterstützung.

Da die Wirkfaktoren-Forschung von Greif noch detaillierter vorgestellt wird (vgl. Kapitel 2.3.2, S. 55 ff.), wird an dieser Stelle nicht näher darauf eingegangen.

Mit Blick auf die Dimension «Kundenmerkmale und -verhalten» nennt Greif aufgrund empirischer Befunde «Offenheit», «Reflexivität», «zunehmender Optimismus», «zunehmende Selbstwirksamkeit», «Selbstmotivierung» und «Ausdauer» als erfolgsrelevant.

\section{Ergebnisse}

Hinsichtlich der Wirksamkeit von Coaching hält Greif (2016, S. 174) einerseits fest, dass Coaching wirkt. Die Effekte der bisher untersuchten Coachings scheinen in Anlehnung an die Auswertung von de Haan und Duckworth (2012) aber nicht sonderlich stark und geringer als Psychotherapie-Effekte, die in RCT-Studien nachgewiesen wurden. Greif ist zudem der Ansicht, dass sich die Wirkung von Coaching noch steigern lässt.

Im Anschluss an die Erkenntnis, dass Coaching wirkt, verweist Greif (2016, S. 174) zum einen auf «allgemeine Coaching-Ergebnisse», d.h. Ergebnisse, die in wirksamen Coachings generell auftreten. Dazu zählen die in RCT-Studien nachgewiesenen Effekte wie «Erhöhung des Zielerreichungsgrads», der «Kundenzufriedenheit», der «Verringerung des negativen Befindens», einer «Verbesserung des allgemeinen Wohlbefindens» und «Steigerung der Selbstwirksamkeitsüberzeugung».

Mit Bezug auf «spezifische Coaching-Ergebnisse» weist Greif (ebd., S. 174f.) auf die Erkenntnisse hin, die Theeboom et al. (2013) in einer neueren Metaanalyse zur Wirksamkeit von Business-Coaching zusammengetragen haben. Mit Bezug 
auf die Auswertung von 18 Untersuchungen, davon acht RCT-Studien, sechs Studien mit nicht randomisierten Vergleichsgruppen und vier Studien mit einer Vorher-nachher-Messung, stellen Theeboom und seine Kollegen und Kolleginnen trotz eines generellen Mangels an objektiven Ergebnisdaten zu den folgenden Dimensionen positive Effekte fest:

- Verhalten/Fertigkeiten,

- Wohlbefinden,

- Coping bei gegenwärtigen oder zukünftigen Arbeitsanforderungen und Stressoren,

- Einstellungen zur Arbeit und Organisation,

- zielorientierte Selbstregulation.

Im Bereich Coping sind die Effekte am schwächsten $(\mathrm{g}=0,43)$, bei der Einschätzung des Zielerreichungsgrades am stärksten $(g=0,74)$. Ähnlich wie in der Metaanalyse von Theeboom et al. (2013) werden auch in der aktuelleren Metanalyse von Jones, Woods und Guillaume (2015), die auf der Auswertung von 17 Studien basiert, durchweg positive Wirkungen hinsichtlich dieser Ergebnisdimensionen (Greif 2016, S. 175) deutlich [eigene Übersetzung]: «Affekt-bezogene Ergebnisse» (Einstellungen, motivationale Ergebnisse usw.), «Fähigkeiten-orientierte Ergebnisse» (Führungsfähigkeit, Kompetenzen usw.) und «Individuum-bezogene Ergebnisse» (Ergebnisse Einzelner, von Teams und der Organisation, finanzielle Ergebnisse, Zielerreichung usw.). Der stärkste Effekt bezieht sich auf die Ebene der «Individuum-bezogenen Ergebnisse» $(\mathrm{g}=1,24)$, der geringste auf die Ebene der «Fähigkeiten-bezogenen Ergebnisse» $(\mathrm{g}=0.28)$.

Eine weitere Metaanalyse kommt zum Schluss, dass Coaching sehr schwache positive Effekte auf das Wohlbefinden der Kundinnen und Kunden (Stresserleben, Wohlbefinden/Glück) hat und etwas stärkere auf die Zielerreichung (Sonesh et al. 2015).

Mit Bezug auf die im Evaluationsmodell erwähnten langfristigen Ergebnisdimensionen weist Greif lediglich darauf hin, dass dazu kaum Studien vorliegen (Greif 2016, S. 173).

\section{Würdigung des Evaluationsmodells von Greif}

Die Darstellung des Forschungsstands nach Greif macht deutlich, dass in Bezug auf Coaching bereits eine Fülle an empirisch gestütztem Wissen über Bedingungs-, Verhaltens- und Ergebnisvariablen vorliegt. Greifs großes Verdienst liegt sicherlich darin, dass er eine Vielzahl wissenschaftlicher Studien zu Coaching und damit verbundene Erkenntnisse und Ergebnisse in die Entwicklung eines umfassenden Evaluationsmodells einfließen ließ, das im deutsch- und englischsprachigen Raum 
großen Anklang findet und fortwährend weiterentwickelt wird. Die positive Resonanz, die das Modell auslöst, hängt neben seiner wissenschaftlichen Fundierung sicherlich auch damit zusammen, dass es einen breiten Blick auf Coaching erlaubt und eine Vielzahl von Variablen sichtbar macht, die für den Erfolg von Coaching bedeutsam sind respektive diesen anzeigen. Darüber hinaus ist das Modell in der Coaching-Praxis äußerst anschlussfähig, da es etwa Auskunft gibt über erfolgsrelevante Verhaltensweisen von Coaches. Die sogenannten Wirkfaktoren sind im wissenschaftlichen Coaching-Diskurs generell und in zahlreichen empirischen und theoretischen Coaching-Arbeiten wichtiger Referenzpunkt (vgl. z.B. Bachkirova et al. 2015; Berninger-Schäfer 2015; Geißler 2016; Geißler et al. 2014; Möller \& Kotte 2011; Schreyögg 2015). Außerdem werden sie auch in adaptierter Form weiter untersucht und in Coaching-Weiterbildungen aufgrund ihres didaktischen Werts eingesetzt (vgl. z. B. Behrendt 2012; Behrendt \& Greif 2018).

\section{Kritische Diskussion und Ausblick auf die ergebnisorientierte Coaching-Forschung}

Obschon die Coaching-Forschung spür- und sichtbar Fortschritte gemacht hat, mangelt es nicht an Kritik. Es wird moniert, dass diese Forschung theoretisch und methodisch auf einem noch instabilen Fundament stehe (Kotte et al. 2016, S. 6). Für die ergebnisorientierte Coaching-Forschung fordern Möller und Kotte (2011) eine generelle Verbesserung der Qualität der Forschungsmethoden, um die teils inkonsistenten Forschungsbefunde zu überwinden. Konkret kritisieren die beiden Autorinnen, Stichproben seien oft zu klein, um auf solcher Grundlage verallgemeinerbare Aussagen zur Wirksamkeit von Coaching machen zu können. Weiter monieren sie, dass es zur Untersuchung der Wirksamkeit von Coaching zu wenig Langzeit- und kaum randomisierte Kontrollgruppenstudien gebe. Die Kompetenzen und Arbeitsweisen der Coaches, deren Coachings untersucht würden, seien zudem sehr heterogen und Vergleiche deshalb problematisch. Weiter seien Coachings, die mit professionellen Coaches - anstelle von wenig geschulten Studenten und Studentinnen - und realen Klienten und Klientinnen stattfänden, eher die Ausnahme. Möller und Kotte thematisieren ferner, dass Messungen von Verhaltensveränderungen häufig auf Selbstbeschreibungen der Kundinnen und Kunden, Coaches oder involvierter Drittpersonen (z.B. Vorgesetzte, Arbeitskolleginnen) basieren. Deutlich aufwendigere und realitätsnähere Untersuchungen, die auf (Verhaltens-)Beobachtungen basierten, existierten dagegen fast keine. Abschließend weisen die beiden darauf hin, dass es zu wenig koordinierte Forschung im Sinne universitärer Forschungsprogramme gebe, mittels deren auch aufwendige und ausgeklügelte Forschungsprojekte über längere Zeit betrieben werden könnten. 
Anschließend an die eben dargelegte Kritik und hinsichtlich einer weiteren Professionalisierung von Coaching braucht es somit hochwertig durchgeführte Forschung, in der nachgewiesen wird, dass professionell durchgeführtes Coaching gute Ergebnisse erzielt. Solche Nachweise müssen auf robusten Untersuchungen im Sinne randomisiert kontrollierter Studien erfolgen, idealerweise in Konkurrenz zu alternativen Interventionen wie etwa Trainings oder virtuell durchgeführten Coachings. Das bedeutet aber auch, dass beobachtbare Verhaltensdaten durch professionell geschulte Forscherinnen und Forscher erhoben und ausgewertet werden müssen, etwa im Sinn der Beobachtung sich verändernder Kompetenzen von Coachees. Wichtig wird weiter sein, dass sich Studien vermehrt an den Ergebnissen aktueller Metaanalysen (vgl. etwa Jones et al. 2015; Theeboom et al. 2013) ausrichten, um dem Kriterium der Vergleichbarkeit gerecht zu werden. Viertens müssen Wirksamkeitsstudien häufiger mittel- und langfristige Ergebnisse untersuchen, um auch die Nachhaltigkeit von Coaching nachzuweisen. Fünftens braucht es Untersuchungen zu qualitativ sehr hochwertigen Coachings mit langjährig erfahrenen und anspruchsvoll zertifizierten Coaches sowie Kunden und Kundinnen mit echten und anspruchsvollen Anliegen (z.B. komplexe Konfliktsituationen in oberen Führungsetagen).

Mit diesen Empfehlungen verbunden ist eine große Schwierigkeit, nämlich die, dass sich Coaches nicht gerne bewerten lassen und es schwierig ist, zu hochwertigen Coaching-Daten zu gelangen (Greif 2016). Dieser Umstand darf aber nicht davon abhalten, in Kooperation mit der Praxis eine noch hochwertigere Coaching-Wirksamkeitsforschung anzustreben. Den Coaching-Verbänden kommt dabei eine große Bedeutung zu: entsprechende Forschungszugänge zu schaffen, da ihre Position sie dazu prädestiniert, als Vermittler zwischen Forschung und Praxis zu fungieren.

\section{Kritische Diskussion der prozessorientierten Coaching-Forschung}

Im Vergleich zur ergebnisorientierten Coaching-Forschung ist die prozessorientierte Coaching-Forschung noch deutlich weniger weit fortgeschritten.

Analog zur Kritik an der ergebnisorientierten Coaching-Forschung ist zu sagen, dass sich auch die prozessorientierte Coaching-Forschung vermehrt auf die Analyse faktischer Coaching-Verläufe stützen und die herkömmliche Interviewforschung im Sinne der Erhebung und Analyse subjektiver Perspektiven und Einschätzungen von Klientinnen, Klienten und Coaches in dieser Weise sinnvoll ergänzen sollte (vgl. dazu auch die Kritik am Forschungsansatz von de Haan et al., Kapitel 2.3.2, S. $63 \mathrm{f}$.). Um sich das Prädikat «hochwertig» zu verdienen, sollte darüber hinaus die Coaching-Prozessforschung auch auf professionell durchgeführten Coachings gründen, die mit hochqualifizierten und erfahrenen Coaches und mit Klienten und Klientinnen mit realen und anspruchsvollen Anliegen durchgeführt werden. 
Das wohl größte Problem der gegenwärtigen Prozessforschung liegt darin, dass das den entsprechenden Studien zugrunde gelegte und so forschungsleitende Verständnis von Coaching-Prozessen deren tatsächlicher Komplexität und Dynamik oft nicht gerecht wird. Aus diesem Grund kann auch von einem «unterkomplexen» Verständnis gesprochen werden, das dem Forschungsgegenstand Coaching nicht vollständig Rechnung trägt. Entsprechend geht es im Sinne einer übergeordneten Handlungsempfehlung inskünftig darum, Coaching-Prozesse noch adäquater zu erforschen und damit - gerade auch für die weitere Professionalisierung von Coaching - wichtige Erkenntnisse zum Verlauf und zur Dynamik erfolgreicher Coaching-Prozesse zu generieren. Auf der Grundlage einer so ausgerichteten Coaching-Prozessforschung wird es im Sinne der Innovationsfunktion von Forschung (Greif 2011) besser möglich, in der Kooperation von Praxis und Wissenschaft effizientere und leistungsfähigere Coaching-Ansätze zu entwickeln.

\subsubsection{Kritische Diskussion der innerhalb der Coaching-Forschung verwendeten Vorstellung von Coaching-Prozessen}

Als Ausgangspunkt für unser Plädoyer zugunsten einer differenzierteren Coaching-Prozessforschung wird nun erst einmal das Prozessverständnis, das dem Evaluationsmodell von Greif zugrunde liegt, diskutiert - dies, weil es sich dabei um eine in der Coaching-Forschung verbreitete Vorstellung von Prozessen handelt, die aber noch deutlich zu wenig differenziert ist. Nicht zuletzt geht es auch darum, mit Blick auf die aktuelle Coaching-Prozessforschung wichtige Forschungsdesiderate sichtbar zu machen.

Zur Erinnerung noch einmal der Hinweis, dass Greif in seinem Evaluationsmodell zur Dimension «Coaching-Prozess» die Unterdimensionen «Coaching-Beziehung», «Verhalten des Coaches» und «Merkmale und Verhalten der Kunden und Kundinnen» (vgl. Tabelle 2.5) unterscheidet.

Im Sinne einer Würdigung gilt es zunächst festzuhalten, dass unter der Dimension «Coaching-Prozess» - unterteilt in die genannten drei Unterdimensionen eine Vielzahl von Merkmalen berücksichtigt wird, die unbestritten in einem unmittelbaren Zusammenhang mit dem Verlauf und der Qualität eines Coachings stehen. Neben den Unterdimensionen «Coaching-Beziehung» und «Merkmale und Verhalten der Kunden und Kundinnen» ist insbesondere die Unterdimension «Verhalten des Coaches» hervorzuheben, da es sich dabei - im Sinne der Handlungssteuerung von Coaches - um ein direkt von ihnen beeinflussbares Potenzial han- 
delt, dies vor allem im Unterschied zur Unterdimension «Merkmale und Verhalten der Kunden und Kundinnen».

Tabelle 2.5 Drei Dimensionen eines Coaching-Prozesses nach Greif (Quelle: Greif 2016, S. 169)

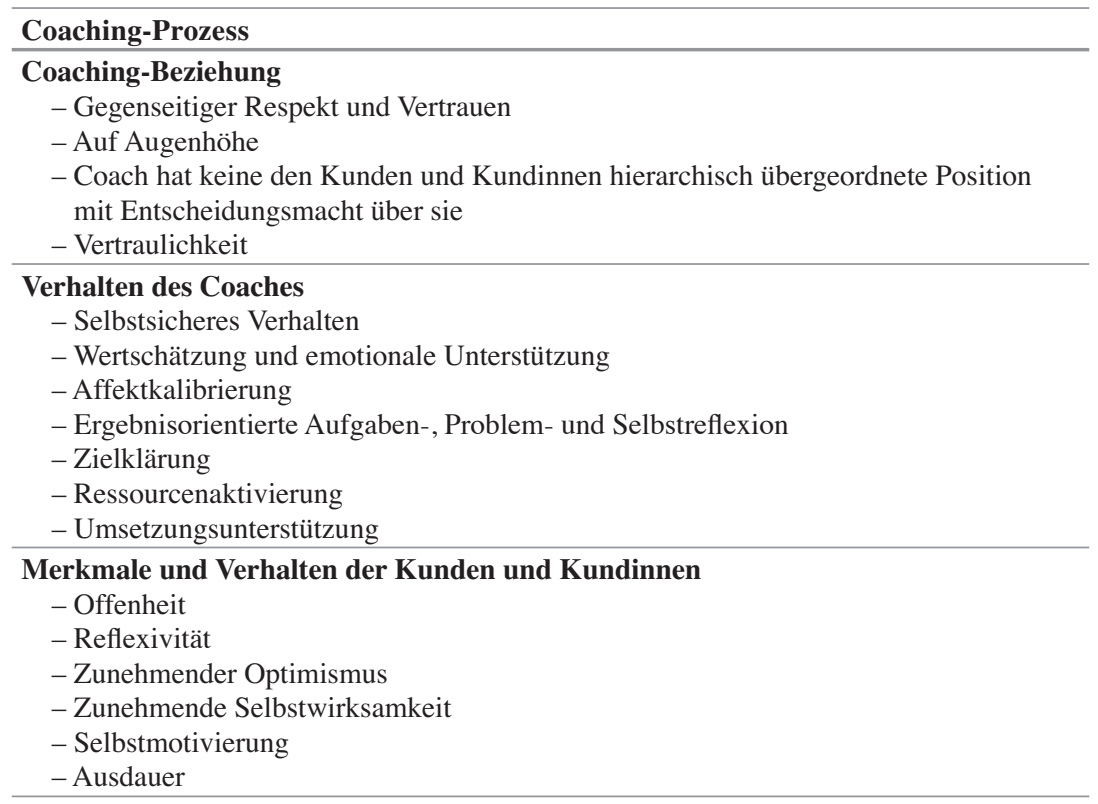

Die Auseinandersetzung mit der Wirkfaktoren-Forschung (vgl. dazu auch Kapitel 2.3.2, S. 55 ff.) macht zum einen deutlich, welche Faktoren im Sinne des Coach-Verhaltens für die erfolgreiche Steuerung von Coaching-Prozessen grundsätzlich von zentraler Bedeutung sind. Darüber hinaus bildet sie auch eine wichtige Grundlage für zwei zentrale Forschungsdesiderate, die beide, insbesondere das zweite, in unmittelbarem Zusammenhang mit der Kritik im Sinne des beschriebenen, gewissermaßen unterkomplexen Coaching-Prozessverständnisses stehen.

So muss erstens darauf hingewiesen werden, dass die praxisnahen Wirkfaktoren, als Beispiel etwa die «Wertschätzung und emotionale Unterstützung» oder die «Ressourcenaktivierung», recht allgemein und abstrakt beschrieben werden, wie das folgende Zitat verdeutlicht: 
«Ein Beispiel ist wertschätzendes und emotional unterstützendes Verhalten des Coaches, anscheinend ein grundlegender Wirkfaktor. Um Ergebnisse zu erzielen, ist es förderlich, wenn der Coach den Klient/innen gezielt hilft, ihre Ressourcen zu erkennen und zu aktivieren (methodische 〈Hilfe zur Selbsthilfe〉), und wenn er systematisch, gegebenenfalls über einen längeren Zeitraum, ihre Umsetzungsversuche unterstützt.» (Greif 2016, S. 178)

Auch wenn Greif (2015b, S. 62) dies in einer aktuellen Darstellung anhand von einzelnen Transkriptbeispielen konkreter illustriert (für eine entsprechende Übersicht vgl. Kapitel 2.3.2, S. 56), bleibt bis zu einem gewissen Grad offen, wie die Wirkfaktoren etwa auf der Ebene einzelner Sprechakte konkret umgesetzt werden. Derartiges Wissen als Ergebnis einer entsprechenden Prozessforschung wäre gerade für praktizierende Coaches im Sinne einer sehr konkreten Handlungsorientierung durchaus interessant - ein Forschungsdesiderat, auf das auch die Linguistin Graf (2012, S. 153 f.) hinweist und das sie wie folgt verdeutlicht (ebd., S. 154):

«Zum anderen kann die Sprachwissenschaft im Zusammenhang mit in anderen Disziplinen etablierten Erfolgsfaktoren für Coaching wie zum Beispiel Wertschätzung und Unterstützung, Zielklärung oder Evaluation im Verlauf(vgl. Greif 2008, S. 277) als Weiterführung die konkrete kommunikative Realisierung dieser Erfolgsfaktoren unter die linguistische Lupe nehmen und sich folgenden Fragen widmen: In welchen kommunikativen Strategien und sprachlichen Formen zeigen sich Wertschätzung und Unterstützung? Wie sehen die kommunikativen Muster im Rahmen einer erfolgreichen Zielklärung aus? Welcher kommunikativen Schritte bedarf es, und in welcher Reihenfolge finden sie idealerweise statt?»

Ausgangslage für ein zweites Forschungsdesiderat ist der Umstand, dass es sich bei Greifs Wirkfaktoren, die den Verhaltensweisen von Coaches zugeschrieben werden, de facto um komplexe Interaktions- und im Sinne des konstruktivistischen Paradigmas eigentlich um Ko-Konstruktionsprozesse - von Coach und Kunde - handelt. Eine erfolgreiche Zielklärung etwa, wie dies im Analyseteil der vorliegenden Arbeit noch gezeigt wird, ist in aller Regel das Ergebnis eines komplexen Zusammenspiels beider Akteure. Deshalb scheint - auch wenn die Prozesssteuerung in einem Coaching dem Coach obliegt - die Frage berechtigt, ob es reicht, komplexe Ko-Konstruktionsprozesse einseitig dem Coach-Verhalten zuzuordnen. Entsprechend muss auch die quantitativ-empirische Wirkfaktoren-Forschung von Greif kritisch hinterfragt werden, da damit Coach-Verhaltensweisen - sprich deren statistische Bedeutung für die finalen Ergebnisse eines Coachings - als Wirkfaktoren untersucht werden, ohne dass die Reaktionen 
der Kundinnen berücksichtigt werden, obschon die Beurteilung des Erfolgs und Nicht-Erfolgs einer Zielklärung damit unmittelbar zusammenhängt.

Durch dieses Ausblenden des Interaktionsgeschehens geht ein wichtiger Aspekt bei der Untersuchung von Coaching-Prozessen verloren, ein Aspekt, der in besonderem Maße den komplexen und dynamischen Charakter von Coaching-Prozessen wiedergibt. So meint Greif selbst an anderer Stelle:

"Es ist sehr wichtig, dass sich der Coach darauf adäquat einstellen kann und eine individuell angepasste Unterstützung ermöglicht. Um die Interaktionsprozesse und Ergebnisse von Coachings zu analysieren, benötigen wir Modelle und Methoden, die nicht nur das Verhalten von Coaches, sondern auch das Verhalten von Kunden und das systemische Zusammenwirken von Coach und Kunde in den Blick nehmen.» (Greif \& Schubert 2014, S. 63)

Fügt man dem hinzu, dass die Dynamik eines Coaching-Prozesses - bezogen auf den gesamten Coaching-Verlauf, der in der Regel mehrere Sitzungen umfasst weit über einzelne Teilaufgaben wie Zielklärung hinausgeht, dass ein Coaching ein höchst komplexes Zusammenspiel bei der Bearbeitung einer Vielzahl von Teilaufgaben ist, wird die Kritik an der gegenwärtigen Coaching-Prozessforschung noch deutlicher.

\subsubsection{Erste Eingrenzung des Forschungsdesiderats}

In den vorangehenden Abschnitten wurde eine erste Vorstellung davon vermittelt, an welchem Punkt sich die gegenwärtige Coaching-Forschung ungefähr befindet. In der Auseinandersetzung mit dem Prozessverständnis, das dem Evaluationsmodell von Greif zugrunde liegt - und insbesondere mit der damit verbundenen Unterdimension «Verhalten des Coaches»-, wurde implizit auf ein der vorliegenden Studie übergeordnetes Forschungsdesiderat hingewiesen. Dieses besteht darin, die bis dato kaum erforschten, für den Erfolg eines Coachings aber zentralen Ko-Konstruktionsprozesse von Coach und Kunde zu untersuchen, die für den Erfolg eines Coachings von eminenter Bedeutung sind.

In den nun folgenden Ausführungen wird - das Prozessverständnis von Greif (2016) gewissermaßen aktualisierend - ein Vorschlag für die Erweiterung der prozessorientierten Coaching-Forschung skizziert. Dieser Vorschlag bildet dann die Grundlage für die weitere Präzisierung des Forschungsdesiderats (vgl. Kapitel 2.3.3), dem in der vorliegenden Arbeit im zweiten Teil Rechnung getragen wird. 


\subsection{Das «Change Process Research»-Paradigma der Psychotherapie-Forschung als Ausgangspunkt für die künftige Ausrichtung der Coaching-Forschung}

Aufder Suchenach Inspirationsquellen, wie eine differenziertere Coaching-Prozessforschung aussehen könnte, bot sich die Auseinandersetzung mit der benachbarten Psychotherapie-Forschung an, dies vor allem deshalb, weil diese sich wie die Coaching-Forschung, nur schon deutlich länger, ebenfalls mit der Untersuchung von Prozessverläufen beschäftigt.

Konkreter Ausgangspunkt der nun folgenden Ausführungen bildet das in der Psychotherapie-Forschung beheimatete «Change Process Research»-Forschungsparadigma (Elliott 2010). Ausgewählt wurde es, weil es eine differenzierte Prozessforschung beschreibt, welche die Dichotomie zwischen ergebnis- und prozessorientierter Forschung zu überwinden versucht. Zweitens fiel die Wahl auf dieses Paradigma, weil darin - systematisch und wie im Evaluationsmodell von Greif basierend auf existierenden Untersuchungen - methodisch und inhaltlich-theoretisch verschiedene Formen der prozessorientierten Psychotherapie-Forschung vorgestellt werden, die ohne signifikante Einschränkungen auf den Forschungsgegenstand Coaching übertragen werden können.

Beim «Change Process Research» geht es darum, «die Effekte jener Prozesse $\mathrm{zu}$ identifizieren, $\mathrm{zu}$ beschreiben, zu erklären und vorauszusagen, die therapeutische Veränderung [therapeutic change] mit sich bringen» (eigene Übersetzung) (ebd., S. 123). Ein weiteres Merkmal dieses auf Greenberg (1986) zurückgehenden Forschungsparadigmas ist der Versuch, die erwähnte Dichotomie von Prozessund Ergebnisforschung zu überwinden und zu randomisiert kontrollierten Wirksamkeits- und experimentellen Einzelfallstudien alternative Studiendesigns vorzuschlagen.

Das zentrale Anliegen des «Change Process Research»-Paradigmas besteht somit darin, über den Nachweis kausaler Beziehungen hinaus zu untersuchen, wie Veränderungen und Ergebnisse in einer Therapie entstehen. Elliott argumentiert in diesem Zusammenhang, dass es immer mehr darum gehe, die Natur von Kausalbeziehungen zu beschreiben, da neue Kausalbeziehungen ohne plausible Erklärungen in der Wissenschaft immer weniger Akzeptanz fänden (Elliott 2010, S. 125). In Anlehnung an Haynes und O'Brien (2000) verweist Elliott dabei auf vier zentrale Kriterien, die für einen validen Rückschluss auf Kausalität erfüllt sein müssen, nämlich [eigene Übersetzung aus dem Englischen): 
- Die beiden Variablen sollen ko-variieren («covary»).

- Die erklärende Variable («causal variable») soll die Ergebnisvariable («effect variable») zuverlässig voraussagen.

- Glaubwürdige alternative Erklärungen der beobachteten Kovarianz sollen auf überzeugende Weise ausgeschlossen werden.

- Es braucht eine logische Erklärung für die Kausalbeziehung («logical mechanism»).

Als Beispiel einer logischen Erklärung für das Zustandekommen von Veränderungen nennt Elliott (2010, S. 123f.) die EMDR-Therapie ${ }^{7}$ (Shapiro 1995). Diese Therapieform fand, trotz Wirksamkeitsnachweisen, die sich auf randomisierte Kontrollstudien stützten, erst mit der Entdeckung und Beschreibung biologischer Erklärungen (Christman et al. 2003) breite Anerkennung.

Elliott (2010) beschreibt vier verschiedene Forschungsstrategien, die Erklärungen für Veränderungsprozesse liefern können, das Kernanliegen des «Change Process Research»-Paradigmas. Im «Process Outcome Design» wird die Prognosekraft ausgewählter Prozessvariablen hinsichtlich der finalen Therapieergebnisse untersucht. Auf der Grundlage des «Helpful Factors Design» werden subjektive Perspektiven von Kundinnen und Kunden zu als hilfreich wahrgenommenen Therapieaspekten erhoben und qualitativ ausgewertet. Im Kontext des «Microanalytic Sequential Process Design» werden Interaktionssequenzen von aufeinander folgenden Reaktions- und Verhaltensweisen der Kundinnen und Therapeuten zur Überprüfung bekannter Interventionen untersucht. Das «Significant Event Design» schließlich charakterisiert sich durch einen multimethodischen Zugang, also durch die Kombination qualitativer mit quantitativen Methoden zur Untersuchung «bedeutsamer Therapieereignisse».

Im Folgenden werden diese vier Forschungsstrategien und exemplarische Studien dazu näher vorgestellt.

\subsection{1 «Process Outcome Design»}

Wie angedeutet, werden in «Process Outcome Design»-orientierten Studien statistische Zusammenhänge zwischen Prozessvariablen und finalen Therapieergebnissen ermittelt (Elliott 2010, S. 124). Als Beispiel für solche Prozessvariablen nennt Elliott die «therapeutische Allianz» als Qualität des Vertrauensverhältnisses von Kunde und Therapeutin oder besondere Therapietechniken wie etwa die

$7 \quad$ EMDR $=\ll$ Eye movement desensitization and reprocessing $»$. 
Interpretation von Übertragungen. In Anlehnung an weitere Autoren (Orlinsky, Ronnestad \& Willutzki 2004) geht Elliott davon aus, dass Studien im Sinne des «Process Outcome Design» bereits tausendfach vorliegen und als sehr prominent eingestuft werden.

Entsprechende Studien erachtet Elliott in Anlehnung an Stiles (1996) dann als sinnvoll, wenn es darum geht, Prozessvariablen zu untersuchen, bei denen «mehr» in der Regel auch «besser» ist. Ein Beispiel dafür wäre etwa die bereits genannte «therapeutische Allianz». Als problematisch erachtet Elliott hingegen, dass in diesem Design die Veränderungsprozesse von Kunden und Kundinnen oft nur als Blackbox behandelt werden und damit ein deterministisch-lineares Therapieverständnis suggeriert wird. Komplexen Therapiesituationen, bedingt etwa durch sehr unterschiedliche Ressourcenlagen der Kundinnen oder Kunden, wird das Design in seiner herkömmlichen Form damit nicht gerecht. Als Beispiel führt Elliott die von Rogers (1957) als für den Erfolg einer Therapie als hinreichend bezeichnete Bedingung der «Empathie» an. Diesbezüglich konnte nämlich gezeigt werden, dass Kundinnen und Kunden, die über genügend Resilienz verfügen, auf eine entsprechende Empathiefähigkeit der Therapeuten und Therapeutinnen nicht zwingend angewiesen sind, um von Therapien profitieren zu können, dies ganz im Unterschied zu fragileren Kundinnen und Kunden (Elliott 2010, S. 125).

Trotz dieser und weiterer Kritikpunkte hebt Elliott (ebd., S. 126) im Sinne des «Process Outcome Design» auch Studien hervor, die zu außerordentlich beeindruckenden Ergebnissen geführt haben. In der Studie von Anderson et al. (2009) etwa, die mit 25 Therapeuten und Therapeutinnen und 1141 Kundinnen und Kunden basierend auf einem «multilevel modelling»-Verfahren durchgeführt wurde, konnten therapeutische Effekte und deren Ursachen sehr überzeugend nachgewiesen werden. Mit Blick auf diese Untersuchung und angelehnt an eine Empfehlung von Kazdin (2009) schlägt Elliott denn auch vor, «Process Outcome»-Studien vermehrt unter Berücksichtigung von Variablen durchzuführen, die Auskunft über die Beziehung von Input- und Output-Variablen geben. Darüber hinaus sollten zusätzlich zu großen Fallzahlen in Anlehnung an Stiles, Honos-Webb und Surko (1998) auch die variablen Ressourcenlagen der Kunden und Kundinnen stärker berücksichtigt sowie zu guter Letzt zur Erhebung der untersuchten Variablen präzisere Messverfahren eingesetzt werden.

\subsection{2 «Helpful Factors Design»}

Das qualitative «Helpful Factors Design» beschreibt Elliott (2010, S. 125 f.) als zum quantitativen «Process Outcome Design» konträr. Im Kern des Designs steht 
die Frage, was Klientinnen und Klienten in Therapieprozessen als hilfreich und als nicht hilfreich empfinden. Grundlage dieses Designs sind offen geführte Interviews (Israel et al. 2008; Levitt, Butler \& Hill 2006; Moertl \& von Wietersheim 2008; Stiles et al. 1998). Im «Change Interview» nach Elliott, Slatick und Urman (2001) werden Klientinnen und Klienten etwa gefragt, was sie als hilfreich, nützlich oder wichtig erlebt haben oder worauf sie die von ihnen beschriebenen Veränderungen zurückführen. In neueren Studien wird gefragt, was außerhalb von Therapien als hilfreich empfunden wurde (Mackrill 2008). Teils werden zur Erhebung entsprechender Daten auch Fragebögen eingesetzt. Ein Beispiel hierfür ist der «Helpful Aspects of Therapy»-Fragebogen nach Llewelyn (1988).

Ein wichtiges Ziel derartiger Studien besteht darin, Anhaltspunkte zu finden, an welcher Stelle in aufgezeichneten Therapiesitzungen vertiefende Untersuchungen durchgeführt werden sollen (Elliott et al. 2009). Zudem werden auf der Grundlage solcher Studien auch Kategorien «hilfreicher Faktoren» gebildet. Als Beispiele nennt Elliott die Grounded-Theory-basierten Auswertungen von Rennie, Phillips und Quartaro (1988), die interpretativ-phänomenologische Studie von Smith, Flowers und Larkin (2009) und die als «consensuel qualitative research» deklarierte Studie von Hill et al. (2005).

Weiter verweis Elliott auf Reviews, in denen die Ergebnisse mehrerer solcher Einzeluntersuchungen bilanziert werden (Elliott 2010, S. 127f.). Dazu gehört etwa die Metaanalyse von Greenberg, Elliott und Lietaer (1994), in der auf der Basis von 14 Studien und damit verbundenen 14 Varianten hilfreicher Aspekte folgende Kategorien «hilfreicher Faktoren» gebildet wurden [eigene Übersetzung aus dem Englischen]:

- positives Beziehungsumfeld, z.B. Empathie oder Unterstützung,

- therapeutische Arbeit der Kunden und Kundinnen, z.B. Selbstoffenbarung oder Erkundungen,

- Förderung der therapeutischen Arbeit der Kundinnen und Kunden durch die Therapeuten und Therapeutinnen, z.B. Erkundungen unterstützen oder Feedback geben,

- Veränderung der Kundinnen und Kunden und damit verbundene Wirkungen, z.B. Bewusstsein oder positive Gefühle.

In einer neueren Metaanalyse (Timulak 2007), auf die Elliott (2010) ebenfalls verweist, wurden, basierend auf sieben ausgewerteten Studien zu den unmittelbaren Wirkungen bedeutsamer Therapieereignisse, folgende, nach Häufigkeit geordnete Kategorien entwickelt [eigene Übersetzung aus dem Englischen]: 
- Bewusstsein/Einsicht/Selbstwahrnehmung,

- Bestätigung/Unterstützung/Sicherheit,

- Verhaltensänderung/Problemlösung,

- Erkundung von Gefühlen/Erleben von Emotionen,

- sich verstanden fühlen,

- Empowerment,

- Erleichterung,

- Beteiligung des Kunden, der Kundin,

- persönlicher Kontakt.

Besonders würdigt Elliott (ebd., S. 127), dass im Sinne des «mental health consumer/service user movement» der Perspektive der Kunden und Kundinnen in den beschriebenen Studien besonders Rechnung getragen wird. Im Unterschied zu «Process Outcome Design»-orientierten Studien, in denen Annahmen und Theorien lediglich getestet werden, besteht in Zusammenhang mit den «Helpful Factors Design»-orientierten Studien zusätzlich die Möglichkeit, Theorien zur Veränderung und zur Entstehung von Veränderung zu entwickeln. Als Beispiel nennt Elliott (ebd., S. 128) das von Stiles et al. (1990) entwickelte «Assimilation Model» (vgl. dazu auch Kapitel 2.4.3).

Orientiert an Kritik aus den Kognitionswissenschaften (z.B. Nisbet \& Ross 1980), erscheint in Bezug auf das «Helpful Factors Design» besonders problematisch (Elliott 2010, S. 127), dass Menschen generell und Therapiepatientinnen und -patienten im Speziellen Ursachen von Ereignissen aufgrund von Attribuierungsfehlern oft falsch einschätzen. Entsprechend kommt es vor, das diese die ursächliche Wirkung von Effekten in Therapien vermuten, die Effekte aber in Tat und Wahrheit auf andere Anstrengungen, wichtige Lebensereignisse, Medikamente und manchmal auch auf die durchgeführten Studien selbst zurückgehen.

Ungeachtet dieser Kritik, sieht Elliott (ebd., S. 127) das «Helpful Factors Design» als sehr wertvoll an. Um den problematischen Aspekten des Designs entgegenzuwirken, empfiehlt er (ebd.), den Nachweis kausaler Zusammenhänge in Kombination mit Studien zu erbringen, die auf einem alternativen methodischen Vorgehen basieren. Zur Qualitätssicherung fordert er zudem, erhobene Daten in der Tiefe und mit kritischer Distanz zu analysieren und Interviewerinnen und Interviewer gut auszubilden. Unter Bezug auf die phänomenologische Psychologie empfiehlt er zudem, mit Kundinnen und Kunden in Interviews nur die für sie wichtigsten Inhalte zu erörtern (Wertz 1986) und bei deren Ausführungen sehr aufmerksam zuzuhören. So ließen sich Selbsttäuschungen der Kundinnen oder Kunden besser aufdecken und wahrhafte von unbegründeten Behauptungen besser unterscheiden, meint Elliott (2010, S. 127) mit Verweis auf Churchill (2000). 


\subsection{3 «Microanalytic Sequential Process Design»}

Im Unterschied zum «Helpful Factors Design» werden im «Microanalytic Sequential Process Design» «Turn-to-turn in-session interaction between client and therapist» (Elliott 2010, S. 128) untersucht. Ausgewählte Aussagen der Therapeutinnen und der Kunden werden dazu im Sinne eines fortwährenden Sprecherwechsels mittels vorhandener Kategorien codiert respektive aufgrund entsprechender Ratingskalen bewertet. Im Sinne eines hypothetisch-deduktiven Vorgehens geht es dabei darum, bereits bekannte Zusammenhänge zwischen therapeutischen Interventionen und Veränderungsprozessen der Kunden und Kundinnen zu überprüfen. Als Beispiele verweist Elliott (ebd., S. 128) auf die Studien von Sachse (1992), Wiseman und Rice (1989), Speisman (1959), Frank und Sweetland (1962) und Snyder (1945).

Positiv bewertet Elliott (2010), dass dieses Design drei der vier von Haynes und O’Brien (2000) geforderten Qualitätsanforderungen berücksichtigt, nämlich 1) die Ko-Variation der Reaktionsweisen von Therapeutin und Kunde, 2) das Einhalten der Reihenfolge von erstens therapeutischer Intervention und zweitens darauf bezogenen Reaktionsweisen der Kundinnen und Kunden sowie 3) das Bereitstellen logischer Erklärungen für den untersuchten kausalen Zusammenhang. Kritisch bewertet er dagegen, dass alternative Erklärungen nicht ausgeschlossen werden und die untersuchten Gesprächssequenzen oft nicht mit den finalen Therapieergebnissen in Beziehung gesetzt werden. Weiter bezeichnet er diese Studien als anspruchsvoll und zeitaufwendig und als für die Entdeckung neuer Zusammenhänge wenig geeignet (Elliott 2010, S. 129).

Trotz der Kritik bewertet Elliott diese Forschungsstrategie positiv (ebd.) und schätzt sie als praxisnah und handlungsorientierend ein. Weiter bewertet er positiv, dass kausale Zusammenhänge direkt und genau untersucht werden. Interessant erscheint der Vorschlag, diese Studien mit statistisch ausgeklügelten Vorgehen entsprechend dem «Process Outcome Design» zu kombinieren, wie dies Tschacher und Ramseyer (2009) in ihrer Modellierung von Therapieprozessen im Sinne von «times-series panel analysis» tun.

\subsection{4 «Significant Event Design»}

Als letzte Forschungsstrategie präsentiert Elliott (2010, S. 129) das «Significant Event Design». Darin werden die bereits präsentierten Forschungsstrategien und somit qualitative mit quantitativen Verfahren kombiniert. Inhaltlich geht es darum, bedeutsame Ereignisse im Verlauf von Psychotherapien zu untersuchen 
(ebd., S. 130). Als Beispiele nennt Elliott «Einsichtsprozesse» (Elliott 1984; Elliott et al. 1994), «Empowerment-Prozesse» (Timulak \& Elliott 2003), «Bearbeiten therapeutischer Aufgaben» (Greenberg 1984), «Übergangspunkte» (Brinegar et al. 2006), «schwierige Momente» (Davis et al. 1987), «Beziehungsbrüche» (Safran et al. 1990) und «Missverständnisse» (Rhodes et al. 1994).

Methodisch betrachtet, beruht die in einem ersten Schritt anstehende Identifikation bedeutsamer Ereignisse (Elliott 2010, S. 130) auf dem Einsatz von Fragebögen (z.B. HAT-Fragebogen nach Llewelyn 1988), auf konkreten Beobachtungen (Greenberg 2007) oder videobasierten Interviewmethoden (Elliott \& Shapiro 1988). Die daran anschließende qualitative und vertiefende Analyse des Verlaufs und der Entwicklung der identifizierten Ereignisse erfolgt in der Auseinandersetzung mit dem Datenmaterial zum faktischen Therapieverlauf. Ziel dabei ist, Theorien zur Entstehung und zum Verlauf bedeutsamer Ereignisse zu entwickeln, wie dies etwa Elliott in seiner «Comprehensive Process Analysis» tut (Elliott, Slatick \& Urman 2000). Darüber hinaus geht es auch darum, bestehende Theorien weiterzuentwickeln. Ein Beispiel hierfür ist das «Assimilation Model»von Stiles (2007), das durch Einzelfallstudien ständig weiterentwickelt wurde. Abschließend unterstreicht Elliott, dass die untersuchten Ereignisse oft mit den finalen Ergebnissen von Therapiesitzungen und manchmal auch mit gesamten Therapieverläufen in Beziehung gesetzt werden.

Wegen des umittelbaren Bezugs zu faktischen Therapieverläufen betont Elliott die hohe Praxisnähe solcher Studien. Weiter unterstreicht er, dass durch die Integration unterschiedlicher Methoden auch die Schwächen monomethodischer Vorgehensweisen überwunden werden könnten. Im Unterschied zu «Process Outcome Design»-orientierten Studien, die oft nur Ein- oder Zwei-Faktoren-Modelle berücksichtigen, können - basierend auf diesem Design - reichhaltige und klinisch relevante Modelle zu Veränderungsprozessen und deren Entstehung entwickelt werden (Elliott 2010, S. 130 f.).

Abschließend verweist Elliott (ebd., S. 131) darauf, dass derartige Studien nicht sonderlich verbreitet sind, da sie technisch anspruchsvoll und zeitaufwendig sind. Weiter erscheint es schwierig, solche Studien zu veröffentlichen, da sie oft nur auf kleinen Fallzahlen basieren. Entsprechend eignen sie sich eher als Teil von Forschungsprogrammen und weniger als «one-shot investigations». Als begrüßenswert bewertet Elliott, wenn in solchen Studien mehr noch der gesamte Therapie- und damit verbundene Veränderungsprozess untersucht und darauf aufbauend umfassende Theorien - wie etwa das «Assimilation Model» (Stiles et al. 1990) - entwickelt würden. 


\subsubsection{Zusammenfassende Darstellung der Forschungs- strategien}

Die zentralen Bestimmungsmerkmale der vorgestellten Forschungsstrategien werden hier tabellarisch noch einmal abgebildet (vgl. Tabelle 2.6). In Anlehnung an die Strukturierung nach Elliott (2010) werden dabei die folgenden Dimensionen verwendet:

- Forschungsziele,

- methodologische Position,

- methodische Ansätze,

- Forschungsergebnisse und -produkte,

- Vorteile und Nachteile,

- Empfehlungen für weitere Entwicklungen,

- Studien als konkrete Beispiele. 


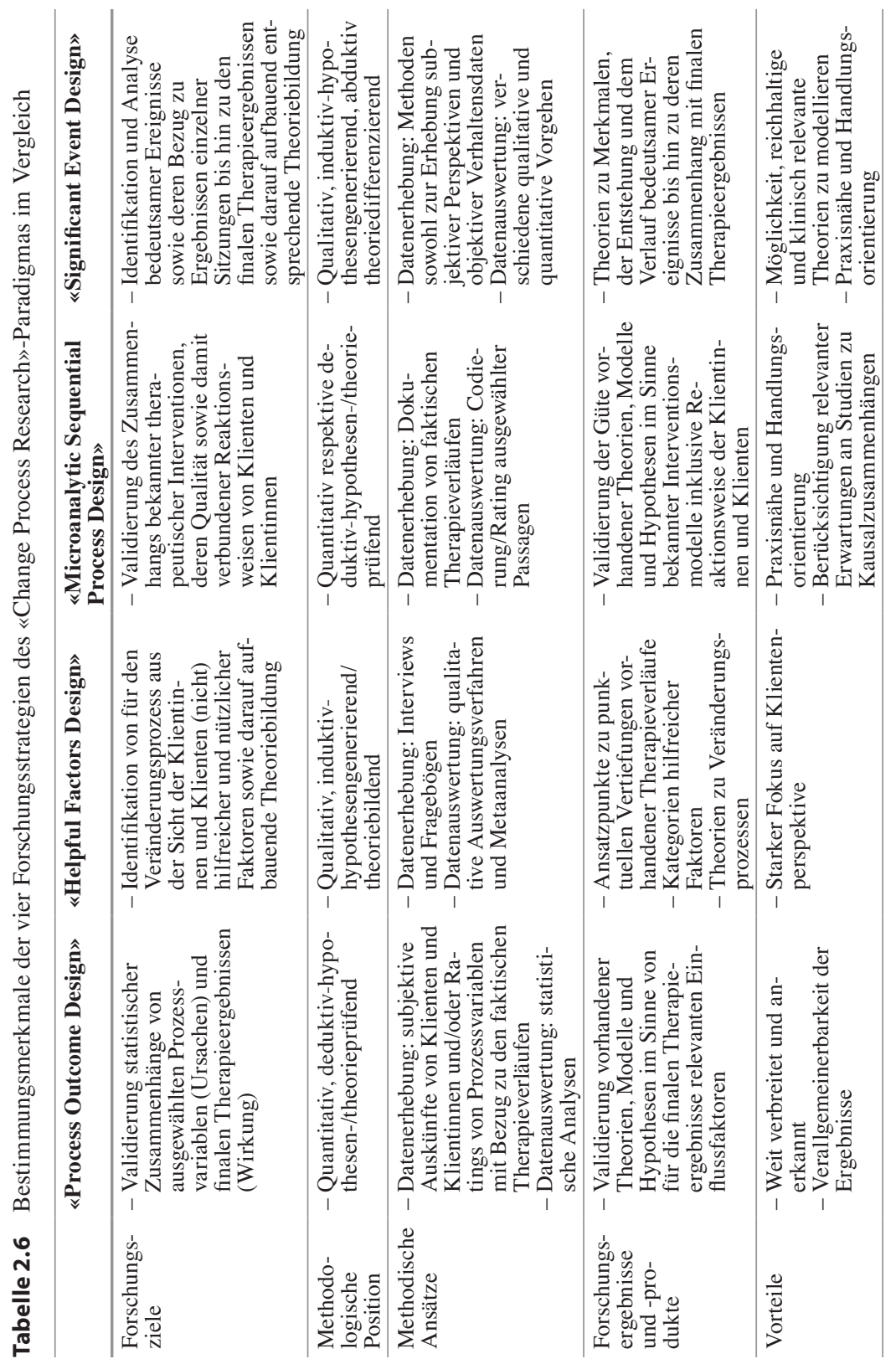




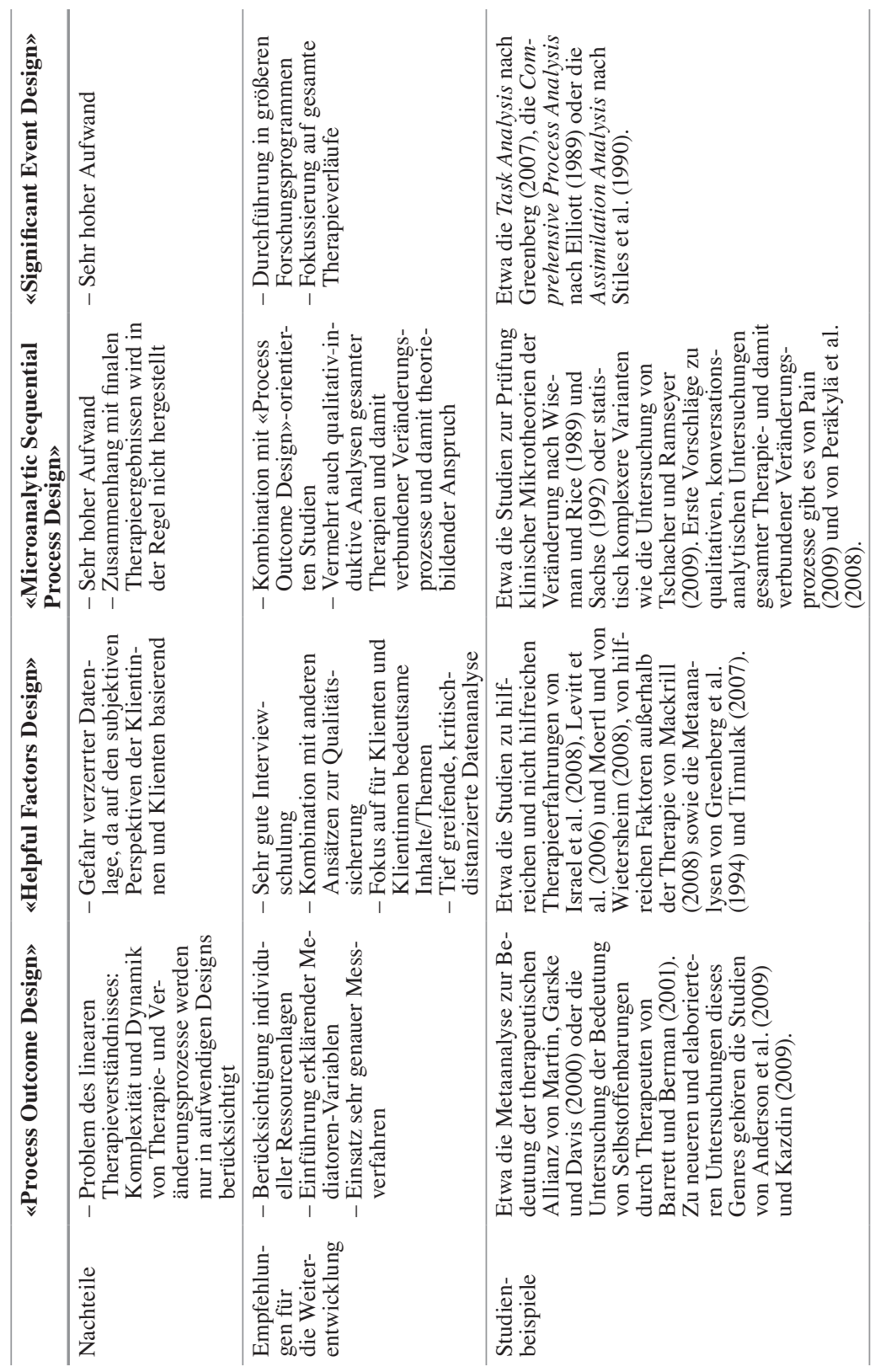




\subsection{Skizzierung einer prozessorientierten Forschungs- landkarte und Verortung darauf bezogener, bereits existierender Coaching-Studien}

Das Ziel des nun folgenden Kapitels besteht darin, das dieser Studie zugrunde liegende, bereits skizzierte Forschungsdesiderat (vgl. Kapitel 2.1.4) weiter zu präzisieren. Der Weg dazu führt erstens über die begründete Übertragung des «Change Process Research»-Paradigmas und der darin enthaltenen Forschungsstrategien auf die Coaching-Forschung. Nach der damit verbundenen Skizzierung einer prozessorientierten Coaching-Forschungslandkarte folgt dann eine zweite Bilanzierung des Coaching-Forschungsstandes. Dazu werden bereits existierende und der skizzierten Forschungslandkarte zuordenbare Coaching-Studien, die in der Darstellung von Greif mehrheitlich unberücksichtigt blieben, präsentiert. Orientiert an dieser Darstellung, wird dann die Frage eingekreist, welches nun noch unbetretene Coaching-Forschungsneuland mit der vorliegenden Arbeit ausgelotet werden soll.

\subsubsection{Eine prozessorientierte Coaching-Forschungslandkarte}

Das «Change Process Research»-Paradigma und die darin enthaltenen Forschungsstrategien stellen eine fruchtbare Grundlage dar für die Erweiterung der von Greif (2016) und anderen (vgl. etwa Ely et al. 2010; Möller \& Kotte 2011) vorgeschlagenen Strukturierungsmöglichkeiten der Coaching-Forschung und ganz besonders der darin enthaltenen Prozessvorstellungen.

Wenn hier nun vorgeschlagen wird, das psychotherapiebezogene «Change Process Research»-Paradigma und die darin enthaltenen Forschungsstrategien auf die Coaching-Forschung zu übertragen, lässt sich dies zunächst damit begründen, dass sich Coaching, wie auch Schiersmann, Friesenhahn und Wahl (2015, S. 12) in Anlehnung an Beck, Brückner und Thiel (1991, S. 11) argumentieren, zu großen Teilen aus methodischen Anleihen bei der Psychotherapie entwickelt hat. Darüber hinaus können beide Formate der personenorientierten Beratung zugeordnet werden, bei denen die Veränderungsprozesse der Klientinnen und Klienten in Bezug auf deren Anliegen im Vordergrund stehen. Obschon signifikante Unterschiede in den jeweiligen Anliegen und Herausforderungen für die Betroffenen bestehen und sich die konkreten Beratungsvorgehensweisen im Einzelnen auch unterscheiden, so handelt es sich aus der Sicht des Verfassers dieser Arbeit bei beiden Formaten um persönliche Formen der Prozessberatung (vgl. Loebbert 2015), in deren Kern das Prinzip der «Hilfe zur Selbsthilfe» steht. In der Psychotherapie werden Pa- 
tienten und Patientinnen darin unterstützt, ihre Selbststeuerung zu verbessern, um psychische Probleme zu überwinden (z. B. Angststörungen oder Depressionen). Im Coaching werden Coachees dabei begleitet, durch die Verbesserung ihrer Selbststeuerung ihre beruflichen oder privaten Ziele zu erreichen (z.B. der Wunsch nach beruflicher Neuorientierung). Primär Prozess- und nicht Fachberatung sind also sowohl Psychotherapie als auch Coaching, weil es in beiden Formaten darum geht, den Betroffenen zu helfen, ihre Selbststeuerung zu verbessern, indem sie wichtige Erkenntnisprozesse durchlaufen und funktionale Handlungsmuster ausbilden, mittels deren es ihnen gelingen kann, ihre Anliegen produktiv und nachhaltig zu bearbeiten, sei dies in Bezug auf die Überwindung von oder das Coping bei Depressionen oder die erfolgreiche Übernahme einer neuen Führungsrolle. Dabei sind geschickt gestellte Fragen zur Verbesserung von Selbststeuerung sicher das Mittel der Wahl. Auf der Basis einer tragfähigen Beziehung können aber auch vorsichtig formulierte inhaltliche Vorschläge und Positionen seitens der Therapeutin oder des Coaches gute Wirkung zeigen.

Diesen formatbezogenen Argumenten folgend, lässt sich die Übertragung der Forschungsstrategien des «Change Process Research»-Paradigmas auf Coaching weiter damit begründen, dass damit nicht beratungsformatspezifische Aspekte vom einem auf das andere Forschungsfeld übertragen werden, sondern forschungsmethodologische Aspekte, die das Verhältnis von Therapie- und Veränderungsprozess der Patienten und Patientinnen betonen. Diese Aspekte lassen sich auch gut auf die Erforschung des Verhältnisses Coaching- und Coacheeprozess übertragen.

In der folgenden Übersicht (vgl. Tabelle 2.7) wird nun die prozessorientierte Forschungslandkarte für Coaching abgebildet. Analog zum «Change Process Research»-Paradigma enthält die Landkarte alle vier oben skizzierten Forschungsstrategien, die mit Blick auf zwei Dimensionen zusammenfassend beschrieben werden. Dazu gehören erstens die Kernideen, die den Forschungsdesigns zugrunde liegen, und zweitens Empfehlungen für deren Weiterentwicklung. Weitere Aspekte, die in der Übersicht zu «Change Process Research» enthalten sind (vgl. Tabelle 2.6), etwa der Vergleich von Vor- und Nachteilen, werden nicht wiederholt, da das Ziel der Coaching-Forschungslandkarte darin besteht, einen unmittelbaren Zugang zu den wesentlichen Kernmerkmalen der einzelnen Designs zu vermitteln. 


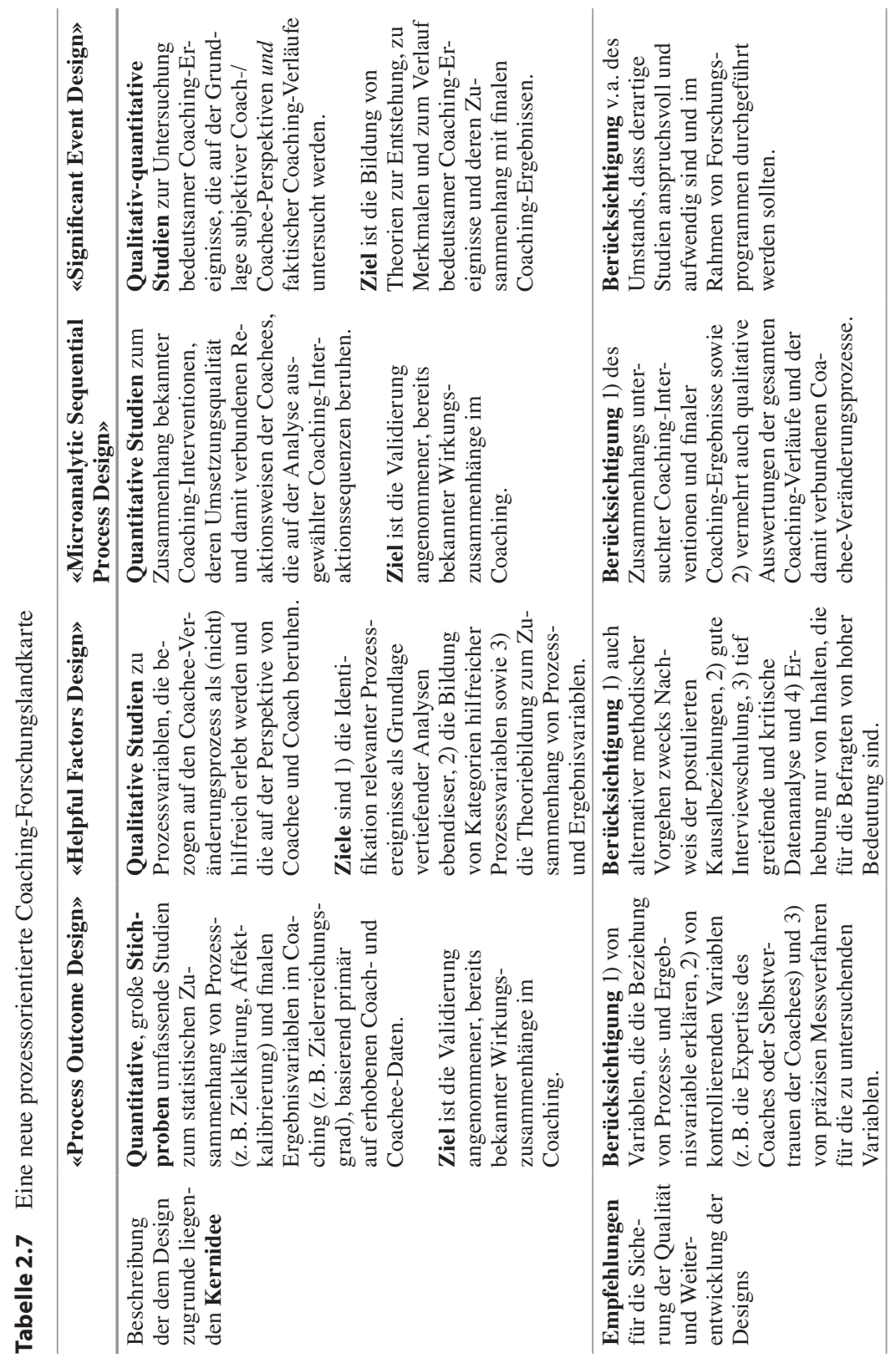




\subsubsection{Der Coaching-Forschungslandkarte zuordenbare Studien}

Bezogen auf die vorgestellte Forschungslandkarte, werden nun Studien vorgestellt, die darin verortet werden können. Dabei handelt es sich um Studien, die in der Darstellung zu Greifs Evaluationsmodell mehrheitlich unberücksichtigt blieben. Dieser Schritt soll verdeutlichen, dass erste Untersuchungen zu den skizzierten Forschungsstrategien bereits existieren, andererseits noch viele Forschungsfragen unbeantwortet sind.

\section{«Process Outcome Design»-orientierte Coaching-Studien}

\section{Die Wirkfaktorenforschung nach Siegfried Greif}

Die an anderer Stelle (vgl. Kapitel 2.1.2 und 2.1.3) bereits behandelte Wirkfaktorenforschung von Greif (Greif 2015; Greif et al. 2010; Greif et al. 2012), in der statistische Zusammenhänge von Prozess- und Ergebnisvariablen gerechnet werden, ist das prominenteste Beispiel für «Process Outcome Design»-orientierte Coaching-Forschung.

Wie erwähnt, schließt der Ansatz an das analytische Ratingverfahren (Greif 2015, S. 64) und Untersuchungen zu Wirkfaktoren in der Psychotherapie. Eine weitere wichtige Grundlage dieser Forschung ist eine Studie von Behrendt (2004), in der die Erkenntnisse zu den psychotherapeutischen Wirkfaktoren auf Coaching übertragen wurden. Entsprechend diesem Forschungszugang, also dieser Form von Ratingverfahren, dürfen die Reaktionen der Kundinnen und Kunden sowie weiterführende Prozesse nicht berücksichtigt werden (ebd.). Deshalb handelt es sich bei den Wirkfaktoren, streng genommen, um Verhaltensweisen der Coaches, bei denen vermutet wird, dass sie eine förderliche Wirkung auf die Coaching-Beziehung und die Coaching-Ergebnisse haben (ebd., S. 65).

Beim angestrebten Wirkungsnachweis geht es konkret darum, die Nutzungshäufigkeit der im Folgenden zusammenfassend abgebildeten Wirkfaktoren nachzuweisen (vgl. Tabelle 2.8) und in einen Zusammenhang mit Coaching-Ergebnisvariablen zu stellen. Dies geschieht über die Identifikation entsprechender Coach-Verhaltensweisen entlang zeitlich einheitlich eingeteilter Beobachtungseinheiten. Zusätzlich zur Nutzungshäufigkeit wird auch die Qualität der identifizierten Coach-Verhaltensweisen mittels Ratingskala bewertet (vgl. Tabelle 2.9). 


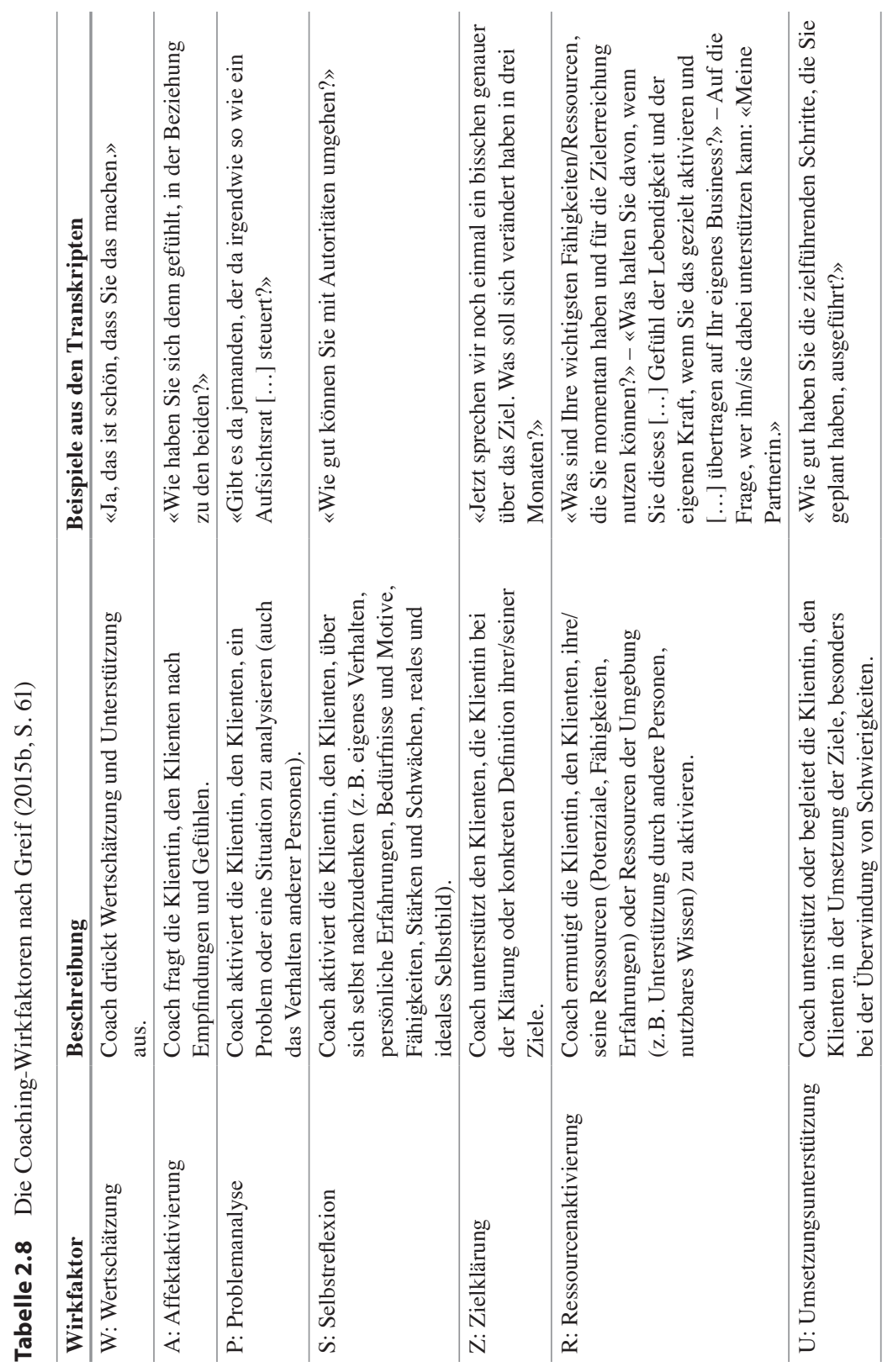


Tabelle 2.9 Die Rating-Ebenen zur Bewertung der Qualität beobachteter Wirkfaktoren nach Greif (2015b, S. 65)

\begin{tabular}{ll}
\hline Rating & Definition \\
\hline 1 & $\begin{array}{l}\text { Vermutlich ist das Verhalten nicht förderlich, oder erforderliches Verhalten } \\
\text { wird unterlassen und nicht gezeigt. }\end{array}$ \\
\hline 2 & $\begin{array}{l}\text { Verhalten wird zum Teil gezeigt, bleibt aber unter dem Standard für professio- } \\
\text { nelles Coaching. }\end{array}$ \\
\hline 3 & $\begin{array}{l}\text { Guter professioneller Standard für Coach-Verhalten wird erreicht, aber Ver- } \\
\text { besserung erscheint möglich. }\end{array}$ \\
\hline 4 & $\begin{array}{l}\text { Ideales Coach-Verhalten (Best Practice), Verbesserungen erscheinen unmög- } \\
\text { lich. }\end{array}$ \\
\hline
\end{tabular}

Das folgende Zitat von Greif (ebd., S. 51), das sich auf die Auswertung einer Erstsitzung eines drei Sitzungen umfassenden Coachings bezieht, veranschaulicht, was für Erkenntnisse und darauf aufbauende Handlungsempfehlungen mit dem Ratingverfahren generiert werden:

\begin{abstract}
«Die Auswertung ergibt, dass der Coach die gesamte Palette der Wirkfaktoren nutzt. In $41 \%$ der Beobachtungseinheiten fördert er die Selbstreflexion, in $34 \%$ die Zielklärung und zeigt in $31 \%$ Wertschätzung und emotionale Unterstützung. Nach den Durchschnittswerten vierstufiger Ratings zur Bewertung der Qualität des Coach-Verhaltens liegt die gezeigte Wertschätzung und emotionale Unterstützung mit 2,56 am höchsten, gefolgt von der Förderung der Selbstreflexion (2,23), Ressourcenaktivierung $(2,27)$ und der Zielklärung $(2,20)$. Nach dem zugrunde liegenden Modell ließe sich die Wirkung des Coachings durch stärkere Verhaltensund Ergebnisorientierung verbessern.»
\end{abstract}

Interessant an der Ergebnispräsentation ist, dass sie die gesamte Palette an Wirkfaktoren und deren bewertete Qualität umfasst. Die Übersetzung der allgemein gefassten Handlungsempfehlungen in umsetzbare Handlungsanleitungen fehlt hingegen.

Ungeachtet möglicher Kritik muss die Wirkfaktoren-Forschung von Greif als pionierhaft und für die Coaching-Forschung und die Coaching-Praxis außerordentlich bedeutsam bewertet werden.

\title{
Das Freiburger Erfolgsfaktorenmodell nach Peter Behrendt
}

Ein mit Greifs Wirkfaktoren-Ansatz eng verwandtes Modell ist das Freiburger Erfolgsfaktorenmodell von Behrendt (Behrendt 2012; Behrendt \& Greif 2018). In seinem Kern liegen die im Folgenden abgebildeten Dimensionen (vgl. Abbildung 2.3). 


\begin{tabular}{|c|c|c|c|}
\hline \multicolumn{4}{|l|}{ Einprägsame Erlebnisse } \\
\hline Wirkung (Kunden) & \multicolumn{3}{|c|}{ Verhalten (Coach) } \\
\hline Vertieftes Verständnis & \multirow{3}{*}{$\begin{array}{l}\text { Ressourcen } \\
\text { aktivieren }\end{array}$} & \multirow{3}{*}{$\begin{array}{l}\text { kooperativ } \\
\text { begleiten }\end{array}$} & \multirow{3}{*}{$\begin{array}{l}\text { Prozessführung } \\
\text { geben }\end{array}$} \\
\hline Gestärkte Motivation & & & \\
\hline Verbesserte Handlungskompetenz & & & \\
\hline
\end{tabular}

Abbildung 2.3 Freiburger Erfolgsfaktorenmodell nach Behrendt (Quelle: Behrendt \& Greif 2018)

Die Abbildung verdeutlicht, welche Verhaltensebenen des Coaches (Prozessführung, kooperativ begleiten, Ressourcen aktivieren) mit welchen Wirkungen beim Coachee (vertieftes Verständnis, gestärkte Motivation, verbesserte Handlungskompetenz) am stärksten korrelieren. Die drei Wirkungen gelten, in Anlehnung an empirische Studien, als maßgebende Bedingungen dafür, dass anvisierte Ziele auch tatsächlich umgesetzt werden.

In Rückgriff auf eine Masterarbeit (Tertocha 2015) und eine eigene Studie (Behrendt 2006) weist Behrendt (Behrendt \& Greif 2018) darauf hin, dass sich professionelle Coaches von Coaching-Anfängern durch eine deutlich klarere Prozessführung, eine kooperativere Begleitung und eine stärkere Ressourcenaktivierung auszeichnen, mit entsprechend positiven Effekten bei den Coachees.

Da sich das Modell von Behrendt insbesondere durch die Komponente «Prozessführung» von Greifs Modell unterscheidet, wird diese Dimension hier vorgestellt. Die «kooperative Begleitung» steht dagegen als Synonym für den im Ansatz von Greif mit «Wertschätzung und emotionale Unterstützung» bezeichneten Wirkfaktor, weshalb darauf nicht eingegangen wird.

Mittels einer «klaren» und «kompetenten Prozessführung» soll ein Coach (Behrendt \& Greif 2018) Coachees Orientierung, Sicherheit und Vertrauen in das Coaching geben. Die Verhaltensweisen, die ein Coach in Anlehnung an das Freiburger Modell dazu realisieren soll, sind (ebd.):

- Coaching-Vorgehen erklären und vermitteln, wie dieses dem Coachee bei der angestrebten Zielerreichung helfen kann;

- Coaching-Prozess strukturieren, damit der rote Faden verstanden wird, erhalten bleibt und bereits Erreichtes verdeutlicht wird;

- persönliche Sicherheit und Kompetenz ausstrahlen, damit der Coachee sich gut aufgehoben und sicher begleitet fühlt.

Wie im Ansatz von Greif werden zur Untersuchung von Coaching-Prozessen nur die Verhaltensweisen der Coaches erfasst. Die Effekte aufseiten des Coachee 
werden jeweils am Ende der einzelnen Coaching-Sitzungen mit einem Fragebogen erhoben. Was im Verlauf des Coachings bei den Coachees in der Interaktion mit dem Coach konkret passiert, wird auch in Behrendts Ansatz nicht untersucht.

Besonders positiv zu würdigen ist bei diesem Ansatz die Erweiterung um die Dimension der Prozessführung, die nach Behrendts Untersuchungen mit den finalen Wirkungen bei den Coachees in einem starken Zusammenhang steht.

\section{«Process Outcome Design»-orientierte Studien der Gruppe um Simone Kauffeld}

Wie die Untersuchungen von Greif und Behrendt können auch drei Studien der Forscherinnengruppe um Simone Kauffeld, deren Ergebnisse in peer-reviewten Journals veröffentlicht wurden, dem «Process Outcome Design» zugeordnet werden. Im Sinne weiterer Beispiele werden diese Studien nun vorgestellt, bezogen auf methodische und inhaltliche Aspekte.

Statistische Untersuchung zum Dominanzverhalten von Coaches: In einer Untersuchung von Ianiro, Schermuly und Kauffeld (2013) konnte mittels eines hypothesenprüfend-statistischen, beobachtungs- und interviewgestützten Verfahrens und orientiert an den sozialpsychologischen Konzepten «zwischenmenschliche Dominanz» und «Unterordnung» («interpersonal dominance and affiliation») nachgewiesen werden, dass in einer ersten Sitzung sehr dominantes Coach-Verhalten mit positiven Einschätzungen der Zielerreichungsgrade durch die Kunden und Kundinnen am Ende eines Coaching-Prozesses korreliert. Je dominanter zudem das Verhalten des Coaches ist («confident behavior»), im Sinne von Vertrauen in das eigene Verhalten/Selbstvertrauen, desto höher wird der Zielerreichungsgrad durch die Kundinnen und Kunden eingeschätzt. Darüber hinaus ließ sich belegen, dass Kunden und Kundinnen dominanter Coaches sich deutlich bereiter zeigen, deren Hilfe und Führung anzunehmen, verglichen mit weniger dominanten Coaches. Nicht bestätigt wurde dagegen die Hypothese, dass dominantes Coach-Verhalten mit positiven Einschätzungen der Beziehungsqualität seitens der Kunden und Kundinnen einhergeht.

Statistische Untersuchung zur Coaching-Beziehung: In einer Untersuchung von Gessnitzer und Kauffeld (2015) wurde der Einfluss von Coaching-Beziehungen auf die finalen Ergebnisse eines Coachings mittels eines hypothesenprüfend-statistischen Beobachtungsverfahrens überprüft. Die Indikatoren für die Beziehungsqualität waren die «Zustimmung zu Zielen und Aufgaben» («agreement on goals and task») und die «gegenseitige Bewertung und Bindung» («apraisal and bonding»).

Die Studie zeigte, dass Bindungsverhalten («bonding») keinen, «durch den Coach signalisierte Zustimmung zu Zielen und Aufgaben» einen negativen und 
«durch den Kunden signalisierte Zustimmung zu Zielen und Aufgaben» einen positiven Effekt auf Coaching-Ergebnisse haben.

Statistische Untersuchung zu empathischem Coach-Verhalten: Die Untersuchung von Will, Gessnitzer und Kauffeld (2016) analysierte mittels eines hypothesenprüfend-statistischen, beobachtungs- und interviewgestützten Verfahrens das Erleben empathischen Coach-Verhaltens durch Kundinnen, Kunden und Coaches. Zweitens wurde der Zusammenhang von beobachtetem empathischem Coach-Verhalten und dem Erleben dieses Verhaltens durch den Kunden oder die Kundin untersucht.

Die Studie zeigt, dass das Erleben empathischen Coach-Verhaltens beim Coach und bei der Kundin, dem Kunden nicht korreliert und beide nach gemeinsam durchgeführten Coaching-Sitzungen diese Dimension unterschiedlich bewerten. Weiter zeigt die Studie im Sinne des zweiten Ziels, dass die Häufigkeit des Paraphrasierens von Gefühlen der Kunden und Kundinnen durch die Coaches einen positiven, das reine Adressieren dieser Gefühle aber keinen Zusammenhang mit dem Erleben empathischen Coach-Verhaltens aufseiten des Kunden oder der Kundin aufweist; dies ergab sich aufgrund der Kundenbewertung ganz am Ende des Coachings.

\section{«Helpful Factors Design»-orientierte Coaching-Studien}

Die einzigen dem Verfasser dieser Arbeit bekannten Studien zu Coaching, die den «Helpful Factors Design»-orientierten Untersuchungen zugeordnet werden können, stellen eine Reihe von Untersuchungen der Forschergruppe um Erik de Haan dar. Gegenstand dieser Untersuchungen sind «critical moments», die mit Interviews und Fragebögen aus der Erlebensperspektive der Coachees und Coaches erhoben und inhaltsanalytisch ausgewertet werden. Aufgrund der inhaltlichen Nähe dieser Untersuchungen zur vorliegenden Arbeit erhält die Darstellung dieser Studien etwas mehr Raum.

\section{Die «critical moments»-Forschung der Gruppe um Erik de Haan}

Seit 2008 untersuchen Erik de Haan und sein Team «critical moments» im Coaching. Theoretischer Bezugspunkt der «critical moments» sind «suboutcomes», die auf die Psychotherapie-Forscher Rice und Greenberg (1984) zurückgehen. Als «suboutcomes» werden Zwischenergebnisse bezeichnet, die in einzelnen Sitzungen entstehen und damit gewissermaßen als Bausteine finaler Therapieergebnisse bezeichnet werden können. De Haan und Nieß definieren, darauf Bezug nehmend und übertragen auf den Kontext von Coaching, «suboutcomes» als «outcomes achieved within the process, from moment to moment, as distinct from outcomes which are generally the result of the process, that is, which can be measured after completing the full coaching assignment» (de Haan \& Nieß 2015, S. 39). 
Forschungsziel von de Haan und seinem Team ist, die subjektiven Erlebensperspektiven von Coaches und Coachees, bezogen auf «critical moments» als Entstehungsmomente von «suboutcomes», zu erheben und zu analysieren. Die Leitfrage, die zu diesem Zweck über verschiedene Studien hinweg den Coachees und Coaches unterbreitet wurde, lautet: «Describe briefly one critical moment (an exciting, tense, or significant moment) with your coach/client. Think about what was critical in the coaching journey, or a moment when you did not quite know what to do» (de Haan \& Nieß 2015, S. 39). Auffällig an der Frage ist ihre offene Formulierung, die - wie später noch erkennbar wird - auch zu eher allgemeinen Ergebnissen führt.

Bezogen auf methodisch relevante Differenzen der insgesamt von de Haan und seinem Team durchgeführten Studien, lässt sich Folgendes festhalten:

- In den ersten Studien von de Haan wurden wenig erfahrene Coaches (de Haan 2008c) und erfahrene Coaches (Day et al. 2008; de Haan 2008b) zu «critical moments» und hinsichtlich ihrer gesamten Erfahrungen mit Coaching befragt.

- In späteren Studien wurden Coaches und Coachees zu «critical moments» und hinsichtlich nur unmittelbar erlebter Coaching-Sitzungen befragt (de Haan et al. 2010a).

- In einer neueren Einzelfallstudie wurde dann ein Coach und ein Coachee hinsichtlich mehrerer Sitzungen zu erlebten «critical moments» befragt (de Haan $\&$ Nieß 2012).

Ein ganz zentrales Ergebnis dieser Studien ist eine auf inhaltsanalytischen Auswertungen der beschriebenen Momente beruhende, zwölf Typen umfassende Kategorisierung von «critical moments», in der die Coachee- und die Coach-Perspektive berücksichtigt wird (vgl. Tabelle 2.10).

Tabelle 2.10 Critical moments coding scheme (Quelle: de Haan \& Nieß 2015, S. 40)

\section{Code Short description of the code}

1 A moment of learning: new insight.

A moment in which new insight was created for the coach and - particularly the client.

2 A moment of learning: new connections or perspectives.

A moment of working through, reflecting, gaining new perspectives and/or making sense of existing material.

3 A change in the relationship in the moment (positive).

$4 \quad$ A change in the relationship in the moment (negative).

$5 \quad$ Significance in doing in the moment (coach-led).

Applying oneself to a unique scripted process such as drawing, visualization, role play, GROW, ... 


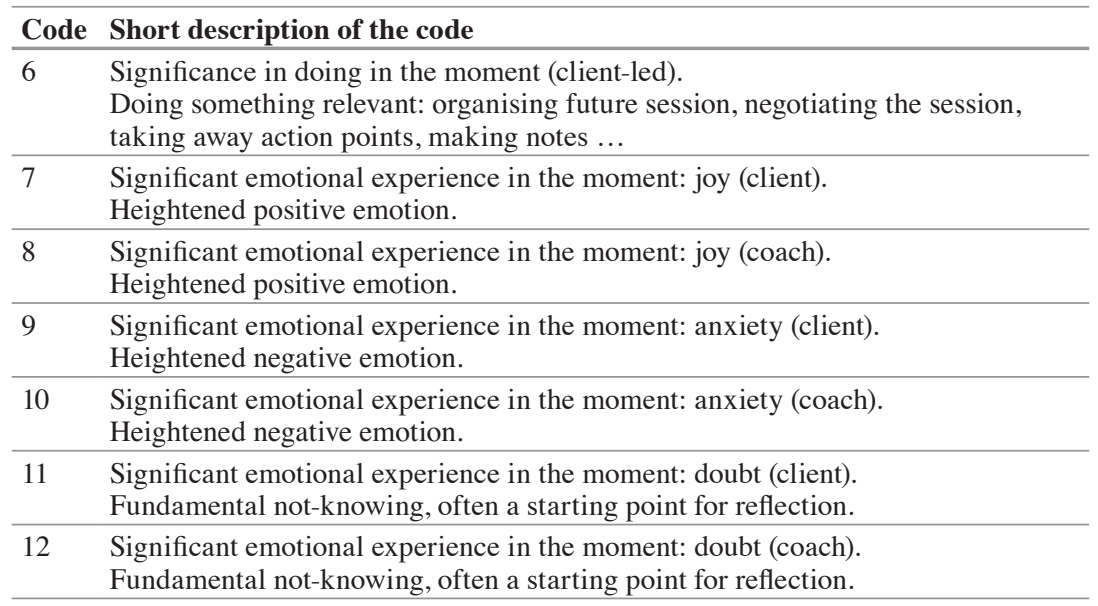

Diese verschiedenen Typen von «critical moments» wurden in vier Kategorien zusammengefasst (de Haan \& Nieß 2015, S. 40):

- «moments of learning» (Code 1,2),

- «moments of relational change in the coaching relationship» (Code 3, 4),

- «moments of significant action» (Code 5,6),

- «moments of significant emotional experience» (Code 7, 8, 9, 10,11, 12).

Weitere zentrale Ergebnisse dieser Untersuchungen sind, dass unerfahrene Coaches oft ihre eigenen Zweifel mit «critical moments» in Verbindung bringen (de Haan 2008c), erfahrene Coaches dagegen eher Ängste (de Haan 2008b). De Haan und Nieß (2015, S. 41) erklären dies damit, dass die Coaches nach ihren allerwichtigsten Momenten («most significant moment») in ihrer Karriere als Coaches gefragt wurden und deshalb eher dramatische oder emotionale Momente ausgewählt haben.

Coachees berichten auf der Basis der gleichen Fragestellung dagegen oft von «new realisations» und «new insights» (de Haan et al. 2010a). Gleiches gilt auch für Coaches und Coachees, die zu unmittelbar erlebten Coaching-Sitzungen befragt wurden. In der erwähnten Einzelfallstudie konnte über den Verlauf mehrerer Sitzungen hinweg weiter gezeigt werden, dass Lernmomente eher zu Beginn, Momente als Ausdruck sich festigender Beziehungen eher gegen Ende des Coachings genannt werden (de Haan \& Nieß 2012). 
Mit Blick auf das Argument von de Haan, dass es zunehmend wichtiger werde, auch die Perspektive der Auftraggeber («sponsors») zu erfassen, da diese in der Regel das Coaching bezahlen (Sherman \& Freas 2004), haben er und sein Team in einer neueren Untersuchung auch diese Perspektive berücksichtigt (de Haan \& Nieß 2015, S. 38 f.). Die wahrgenommene Wirkung von Coaching konnte damit um die Perspektive der Auftraggeber erweitert werden. So beziehen sich Coaches und Coachees am häufigsten auf

- «moments of new realisations»,

- «moments of insight» und

- «coach-led significance in doing in the moment»;

die Auftraggebenden dagegen vor allem auf

- «positive changes in the relationship with the client» und

- «client-led significance in doing in the moment»,

wobei sich Letzteres im Wesentlichen auf die Veränderungen des Coachee-Verhaltens bezieht (de Haan \& Nieß 2015, S. 52).

\section{Diskussion der Untersuchungen zu «critical moments»}

Der Wert der Untersuchungen von de Haan et al. liegt in der Berücksichtigung der Erlebensperspektiven von drei unterschiedlichen Personengruppen, den Coaches, den Coachees und den Auftraggebern. Durch diesen Zugang zu «critical moments» im Sinne von spannungsvollen («exciting»), angespannten («tense») oder wichtigen («significant») Momenten rücken - analog zur Argumentation von Elliott (vgl. Kapitel 2.2.2) - die Perspektiven der unmittelbar betroffenen Personen in den Vordergrund. Darüber hinaus stellt die Kategorisierung der zwölf unterschiedlichen «critical moments», in der positive wie auch negative Erlebensaspekte dieser Momente sichtbar werden, einen zentralen Erkenntnisgewinn dar. Weiter gilt es zu würdigen, dass de Haan et al. mit diesen Studien - im Vergleich gerade etwa zur Wirkfaktorenforschung von Greif - den gewichtigen Umstand deutlich machen, dass nicht erst am Ende einer Sitzung oder eines Coachings Ergebnisse für die Kundinnen und Kunden entstehen, sondern sich diese als Resultat einer schrittweisen Kumulation von in «critical moments» eingebetteten «suboutcomes» ergeben.

Trotz dieser positiven Bewertung der Studien von de Haan et al. lässt sich auch kritisieren, dass die generierten Erkenntnisse sehr allgemein und dass viele Fragen ungeklärt bleiben - so etwa, was für Arten von «new insights» oder «new connec- 
tions, new perspectives» es gibt und wie diese in der Interaktion von Coach und Coachee konkret entstehen. Ähnliche Fragen stellen sich auch zur Entstehung negativer oder positiver Emotionen, die im Zusammenhang mit «critical moments» genannt werden. Eine weitere Differenzierung der erlebten Emotionen und deren Verbindung zu mentalen Prozessen könnten den Erkenntniswert von Studien zu «critical moments» weiter erhöhen.

Mit Blick auf das Konstrukt der «suboutcomes» bleibt im Rahmen der vorliegenden Untersuchungen auch mehrheitlich ungeklärt, wie einzelne «critical moments» im Sinne eines logischen Zusammenhangs mit den finalen Coaching-Ergebnissen zusammenhängen. Offen bleibt ferner die Frage der methodischen Angemessenheit: Inwiefern ist ein Zugang exklusiv über die subjektiven Erlebensperspektiven von Coach und Coachee und ohne Berücksichtigung der faktischen Coaching-Verläufe als angemessen zu bewerten, um «critical moments» zu untersuchen? Gründe für diesen Einwand liegen nicht nur in den ungenutzten Möglichkeiten, auf der Grundlage faktischer Coachings Verlauf und Merkmale entsprechender Momente zu rekonstruieren, sondern darüber hinaus auch in der bereits geäußerten Kritik an herkömmlicher Interview- und Fragebogenforschung. Damit gemeint sind insbesondere die Risiken verzerrter Erlebensperspektiven und sozial erwünschter Antworten (vgl. dazu auch die Kritik von Elliott in Kapitel 2.2.2).

Analog zur Wirkfaktorenforschung von Greif dürfen aber auch die «critical moments»-Untersuchungen von de Haan und Team zweifelsohne als pionierhaft für die gesamte Coaching-Forschung bewertet werden. So ist zu hoffen, dass es weiterführende Untersuchungen zu diesem wichtigen thematischen Fokus geben wird.

\section{«Microanalytic Sequential Process Design»-orientierte Coaching-Studien}

Studien im engeren Sinne des «Microanalytic Sequential Process Design», dessen Ziel darin besteht, bekannte Zusammenhänge von Coach-Interventionen, deren Umsetzungsqualität und den Zusammenhang mit Coachee-Reaktionen zu validieren, gibt es bis anhin gemäß dem Wissensstand des Autors der vorliegenden Studie keine.

Dafür existiert bereits eine Fülle an Forschungsansätzen, welche die exakte Erfassung des Geschehens in einem Coaching zum Ziel haben. Obschon mit diesen Forschungsansätzen eher ergebnisoffen die übergeordnete Frage beantwortet werden soll, was Coaching-Prozesse ausmacht und worin sie bestehen, eignen sie sich grundsätzlich auch zur Validierung entsprechender Wirkzusammenhänge im Sinne des hier diskutierten Forschungsdesigns. Vier solch offene Forschungsansätze werden nun vorgestellt und im Anschluss diskutiert. 


\section{Das sinnerschließende Interpretationssystem zur Erfassung von Coaching als Kommunikationsgattung von Harald Geißler}

Das «sinnerschließende Interpretationssystem» von Geißler (2016), das in Kapitel 1.5.3, S. 18, bereits skizziert wurde und in Kapitel 3.4.2, S. 115 ff., noch ausführlicher vorgestellt wird, wurde in der Auseinandersetzung mit faktischen Coaching-Prozessen und aktueller Coaching-Literatur abduktiv entwickelt. Es erhebt den Anspruch, Coaching-Schulen-unabhängig zu sein. Inhaltlich dient es zum einen der Rekonstruktion des Sinns, der einzelnen Sprechakten von Coach und Coachee zugrunde liegt. Rekonstruiert wird der Sinn dieser - wie Geißler sie nennt - «basalen Einzelhandlungen» oder «Kommunikationsentscheidungen» unter Berücksichtigung der im Folgenden abgebildeten elf Entscheidungsdimensionen (vgl. Tabelle 2.11).

Das übergeordnete Ziel des Interpretationssystems besteht darin, durch die Rekonstruktion aller theoretisch möglichen Kommunikationsentscheidungen von Coaches und Coachees Coaching als eigenständige Kommunikationsgattung zu beschreiben und damit auch von anderen Beratungsformen wie Psychotherapie oder Fachberatung abgrenzen zu können. Als wichtiges qualitatives Ergebnis seiner Forschung rekonstruierte Geißler eine «Entscheidungssystematik» (ebd., S. 195-282), und als zentrales quantitatives Ergebnis errechnete er eine darauf bezogene Nutzungshäufigkeit (ebd., S. 283-355).

Eine wichtige methodische Eigenheit des Interpretationssystems liegt darin, dass die zu erfassenden Kommunikationsentscheidungen durch die Bildung unterschiedlicher Paraphrasen codiert werden, und zwar unter Rückgriff auf die elf weiter unten dargestellten Entscheidungsdimensionen. So werden jeder Kommunikationsentscheidung insgesamt elf Codes zugeordnet und alle diese Entscheidungen damit sehr präzise erfasst. Da dieser Forschungsansatz auf einem paraphrasenbildenden Verfahren beruht, handelt es sich nicht um einen Beobachtungs- oder Ratingansatz, sondern, wie Geißler es selbst beschreibt, um ein «sinnerschließendes Interpretationssystem» ${ }^{8}$.

Der Kern des dem Interpretationssystem zugrunde liegenden Verständnisses von Sprechakten im Sinne basaler Kommunikationsentscheidungen von Coach und Coachee ist die Unterscheidung von illokutionären und propositionalen Aspekten einer Kommunikationsentscheidung. Illokutionäre Aspekte beziehen sich auf die Bestimmung der Beziehung von Sprecher, Angesprochenem und Gesprächsinhalt, propositionale Aspekte dagegen auf die nähere Bestimmung des

8 Diese Bezeichnung geht auf eine mündliche Aussage von Prof. Dr. Harald Geißler während des 4. Internationalen Coaching-Kongresses vom 14./15. Juni 2016 in Olten/ Schweiz zurück. 
Gesprächsinhalts. Weiter kennzeichet das Interpretationssystem, dass es zwischen facilitativen und instruktionalistischen Kommunikationsentscheidungen unterscheidet. Damit gemeint sind Kommunikationsentscheidungen, mittels deren eine Sprecherin den Angesprochenen einerseits anregen kann, etwas Bestimmtes zu tun (= facilitative Kommunikationsentscheidung), zum Beispiel einen Entscheid zu fällen. Andererseits kann ein Sprecher mit seinen Sprechhandlungen der Angesprochenen auch anzeigen, was sie gerade tut (= instruktionalistische Kommunikationsentscheidung), dass sie etwa gedanklich einen gewissen Inhalt fokussiert (ebd., S. 160).

Tabelle 2.11 Elf konstitutive Entscheidungsdimensionen nach Geißler (eigene Darstellung, hergeleitet aus Geißler 2016, S. 198-263)

\begin{tabular}{|c|c|c|c|}
\hline \multirow[t]{2}{*}{ Dimension } & \multirow[t]{2}{*}{$\begin{array}{l}\text { facilitativ vs. } \\
\text { instruktionalistisch }\end{array}$} & \multicolumn{2}{|c|}{$\begin{array}{l}\text { illokutionärer vs. } \\
\text { propositionaler Aspekt }\end{array}$} \\
\hline & & illokutionär & propositional \\
\hline $\begin{array}{l}\text { A: Entscheidungen } \\
\text { bezüglich der Wahl } \\
\text { der rahmensetzenden } \\
\text { Kommunikations- } \\
\text { aktivitäten }\end{array}$ & $\begin{array}{l}\text { Jeder Code ist entweder } \\
\text { facilitativ oder } \\
\text { instruktionalistisch }\end{array}$ & $\checkmark$ & \\
\hline $\begin{array}{l}\text { B: Entscheidungen } \\
\text { bezüglich der Wahl } \\
\text { der Problemlösungs- } \\
\text { aktivitäten }\end{array}$ & $\begin{array}{l}\text { Jeder Code existiert in } \\
\text { beiden Varianten }\end{array}$ & $\checkmark$ & \\
\hline $\begin{array}{l}\text { C: Entscheidungen } \\
\text { bezüglich der } \\
\text { Wahl des sozialen } \\
\text { Ausgangsstandpunkts }\end{array}$ & $\begin{array}{l}\text { Jeder Code existiert in } \\
\text { beiden Varianten }\end{array}$ & $\checkmark$ & \\
\hline $\begin{array}{l}\text { D: Entscheidungen } \\
\text { bezüglich der Wahl } \\
\text { des zeitlichen } \\
\text { Ausgangspunkts }\end{array}$ & $\begin{array}{l}\text { Jeder Code existiert in } \\
\text { beiden Varianten }\end{array}$ & $\checkmark$ & \\
\hline $\begin{array}{l}\text { E: Entscheidungen } \\
\text { bezüglich der } \\
\text { Thematisierungswahl des } \\
\text { Handlungssubjekts }\end{array}$ & $\begin{array}{l}\text { Jeder Code existiert in } \\
\text { beiden Varianten }\end{array}$ & $\checkmark$ & \\
\hline $\begin{array}{l}\text { F: Entscheidungen } \\
\text { bezüglich der } \\
\text { Thematisierungswahl des } \\
\text { Handlungsaspekts }\end{array}$ & $\begin{array}{l}\text { Jeder Code existiert in } \\
\text { beiden Varianten }\end{array}$ & & $\checkmark$ \\
\hline
\end{tabular}




\begin{tabular}{llll}
\hline Dimension & $\begin{array}{l}\text { facilitativ vs. } \\
\text { instruktionalistisch }\end{array}$ & \multicolumn{2}{l}{$\begin{array}{l}\text { illokutionärer vs. } \\
\text { propositionaler Aspekt }\end{array}$} \\
\hline $\begin{array}{l}\text { G: Entscheidungen über die } \\
\text { Bezugnahme auf einen } \\
\text { thematischen Kontext }\end{array}$ & $\begin{array}{l}\text { Jeder Code existiert in } \\
\text { beiden Varianten }\end{array}$ & $\checkmark$ \\
\hline $\begin{array}{l}\text { H: Entscheidungen } \\
\text { bezüglich der zeitlichen } \\
\text { Positionierung des } \\
\text { Kommunikationsinhalts }\end{array}$ & $\begin{array}{l}\text { Jeder Code existiert in } \\
\text { beiden Varianten }\end{array}$ & $\checkmark$ \\
\hline I: $\begin{array}{l}\text { Entscheidungen über die } \\
\text { thematische Bezugnahme } \\
\text { auf Faktisches oder } \\
\text { Mögliches }\end{array}$ & $\begin{array}{l}\text { Jeder Code existiert in } \\
\text { beiden Varianten }\end{array}$ & $\checkmark$ \\
\hline J: $\begin{array}{l}\text { Entscheidungen bezüg- } \\
\text { lich der Bewertung des } \\
\text { Kommunikationsinhalts }\end{array}$ & $\begin{array}{l}\text { Jeder Code existiert in } \\
\text { beiden Varianten }\end{array}$ & \\
\hline K: Entscheidungen, welches \\
$\begin{array}{l}\text { Medium in welcher Weise } \\
\text { genutzt wird }\end{array}$ & $\begin{array}{l}\text { Jeder Code existiert in } \\
\text { beiden Varianten }\end{array}$ & \\
\hline
\end{tabular}

Eine zentrale Erkenntnis, die durch die Untersuchung von Geißler zur Begründung von Coaching als Kommunikationsgattung entstand, ist, dass Coaches im Vergleich zu Coachees deutlich häufiger facilitative Kommunikationsentscheidungen treffen. Das heißt, dass sie konkret häufiger Fragen stellen oder das Gesagte der Coachees spiegeln, womit diese in beiden Fällen angeregt werden, sich darauf zu beziehen (z.B. etwas zu reflektieren oder das Gesagte zu überprüfen). Bei den Coachees ist es gerade umgekehrt. Sie treffen deutlich häufiger instruktionalistische Kommunikationsentscheide. Das heißt, sie vermitteln dem Coach oft fallspezifische Informationen oder offenbaren diesem unmittelbar erlebte Gefühle, getroffene Entscheidungen oder äußern gerade ablaufende Gedankengänge (Selbstoffenbarung) (ebd., S. 299).

Das «sinnerschließende Interpretationssystem» von Geißler ist außerordentlich differenziert und erlaubt deshalb auch eine extrem genaue Bestimmung einzelner Kommunikationsentscheidungen. Aufgrund seiner hohen Komplexität, bedingt durch die Vielzahl an Entscheidungsdimensionen sowie den interpretativen Charakter, eignet sich das Interpretationssystem in seiner Anwendung jedoch eher für Forschungsteams und weniger für einzelne Forschende. 


\section{Die «Q-Methodology», entwickelt von der Gruppe um Tatiana Bachkirova}

Der Forschungsansatz von Bachkirova et al. (2015) wurde im Unterschied zum Ansatz von Geißler im Austausch mit professionellen Coaches entwickelt. Ebenso wird aber der Anspruch erhoben, dass er Coaching-Schulen-unabhängig und zudem auch auf andere Beratungsformate wie Psychotherapie übertragbar sein soll.

Inhaltlich umfasst der Ansatz, der auf einer sogenannten Q-Methodology gründet, aktuell achtzig «Descriptors», die für Coaching-Verläufe typische Merkmale abbilden. Ein erstes Ziel, dem die Forscherinnen bereits nachgingen (ebd.), bestand darin, jene Merkmale zu erfassen, die aus Sicht von Coaches zur Bewertung von Coaching und zur Abgrenzung gegenüber anderen Beratungsformaten relevant erscheinen. Auf der Grundlage noch zu entwickelnder «Descriptor»-Skalen sollen künftig als zweites Forschungsziel auch reale Coaching-Sitzungen damit präzise beschrieben werden (Bachkirova et al. 2015, S. 432, 436).

Aus einer Analyse der «Descriptors» durch Geißler (2016, S. 42-47) geht hervor, dass 43 davon sich im Sinne «basaler Einzelhandlungen» auf das Coach- und Coachee-Kommunikationsverhalten beziehen (die große Mehrheit auf das Coach-Verhalten). Vier «Descriptors» werden hier zur Illustration abgebildet (ebd., S. 43):

- 07 «Coach points out recurrent theme in client's behavior.»

- 08 «Coach points out potential unconscious motives of the client.»

- 60 «Client interrupts coach.»

- 65 «Client suggests his next course of action.»

Einen weiteren «Descriptor»-Typ ordnet Geißler (ebd., S. 44) im Sinne von «Handlungskonglomeraten» dem Coach-Verhalten zu, da Komplexität und zeitlicher Umfang unbestimmt sind. Ähnlich wie bei Greifs Wirkfaktoren stellt sich hier aber die Frage, ob es nicht angemessener wäre, diesen im Folgenden aufgeführten Einzelfall als Interaktionsgeschehen zu fassen:

- 06 «Coach works with the client's apparent defensiveness.»

Zwei weitere «Descriptors» bezieht Geißler auf Handlungsmerkmale der Coaches, die sich nicht auf basale Einzelhandlungen oder Handlungskonglomerate beziehen, sondern auf die gesamte Sitzung (ebd.):

- 50 «Coach takes an active role during the session.»

- 58 «Coach is verbose.» 
45 «Descriptors» bezieht Geißler auf Interaktionsverhalten von Coach und Coachee, wobei sich keiner dieser «Descriptors» auf die gesamte Sitzung bezieht. Im Folgenden dazu zwei Beispiele (ebd.):

- 1 «There is an exploration of the effect of client's choice of words.»

- 2 «There is an exploration of the client's values.»

Vier «Descriptors» stellen Interaktionsverhalten dar und beziehen sich auf die gesamte Sitzung (ebd., S. 45):

- 37 «There is a sense of optimism in the coaching session.»

- 39 «Coach and client appear to be engaged (vs. disengaged).»

- 46 «The session is fast-paced.»

- 47 «The session appears highly structured.»

Ein wichtiges Ergebnis der Untersuchung zu den Vorstellungen von 41 unterschiedlich geschulten Coaches ist, dass ein recht einheitliches Bild darüber existiert, was professionelle Coachings ausmacht und dass dieses maßgebend durch folgende Coach-Fragen bestimmt ist (Bachkirova et al. 2015, S. 447):

- Q41 «Coach asks questions helping the client to elaborate.»

- Q63 «Coach asks questions that appear to open new possibilities for the client.»

Darüber hinaus werden zwei «Descriptors» in den statistischen Auswertungen (ebd., S. 452) als diejenigen identifiziert, die im Kontrast zu anderen Beratungsformaten durch die professionellen Coaches als besonders charakteristisch für Coaching betrachtet werden:

- Q05 «Coach and client explore the deeper meaning of a presenting issue.»

- Q07 «Coach points out a recurrent theme in client's behavior.

Die folgenden «Descriptors» wurden dagegen als für Coaching wenig charakteristisch bewertet (ebd.), womit auch eine mögliche Differenz zur Psychotherapie erkennbar wird:

- Q13 «Coach encourages client to feel more deeply within session.»

- Q06 «Coach works with the client's apparent defensiveness.»

- Q08 «Coach points out potential unconscious motives of the client.» 
Wie erwähnt, wollen Bachkirova et al. auf der Grundlage einer noch anstehenden Entwicklung entsprechender Skalen - über die Erfassung der Vorstellungen professioneller Coaches, was Coaching ausmacht - das «Descriptor»-Set auch noch zur Erfassung konkreter Coaching-Verläufe einsetzen. Ergebnisse dazu stehen noch aus.

Am Ansatz von Bachkirova et al. besonders zu würdigen ist deren Zugang über die Vorstellungen professioneller Coaches hinsichtlich der Frage, wie Coaching im Unterschied zu anderen Beratungsformaten beschrieben und abgegrenzt werden kann. Darüber hinaus erscheint auch als sehr wertvoll, dass in den «Descriptors» sowohl die Coach- als auch die Coachee-Aktivitäten abgebildet werden. Um mit dem Ansatz arbeiten zu können, erscheint es, ähnlich wie beim Ansatz von Geißler, beinahe unumgänglich, in Forscherteams zu arbeiten.

\section{Linguistische Rekonstruktion von Coaching-Verläufen nach Eva-Maria Graf}

Als Grundlage eines weiteren wichtigen Coaching-Prozessforschungs-Ansatzes identifiziert Eva-Maria Graf in ihrer Habilitationsschrift (Graf 2015a) «strukturelle», «thematische» und «interaktive» Charakteristika von Coaching, indem sie neun Coaching-Prozesse hinsichtlich ihrer sequenziellen Abfolge von Gesprächsbeiträgen (= turns) diskursanalytisch untersucht (Graf 2015b, S. 12). Besonders bezeichnend für diesen linguistischen Coaching-Forschungs-Ansatz ist das zugrunde liegende Verständnis von Coaching als professionelles Gespräch, «das von Coach und Klient/in lokal im Hier und Jetzt diskursiv ko-konstruiert wird» (ebd., S. 11). Entsprechend konsequent analysiert Graf die Interaktionsprozesse von Coach und Coachee basierend auf der Vorstellung, dass diese das Coaching-Geschehen kommunikativ ko-konstruieren.

Als wichtiges Ergebnis ihrer linguistischen Analysen rekonstruiert Graf als Strukturmerkmale vier «kommunikative basale Aktivitäten», die im Unterschied zu gängigen Phasenmodellen der Praxisliteratur den schleifenartigen und komplexen Charakter von Coaching als sozialer Interaktion beschreiben. Damit will Graf die «relationale Dynamik» und «diskursive Komplexität» von Coaching-Prozessen abbilden (ebd., S. 213).

Die basalen Aktivitäten sind (ebd.):

- «Definieren der Situation»,

- «Gestalten der Beziehung»,

- «Ko-Konstruieren der Veränderung»,

- «Evaluieren des Coachings». 
Gemäß Graf sind für Coaching - gerade in Unterscheidung zu anderen Gesprächstypen - die auf einer tieferen Abstraktionsebene situierten «kommunikativen Aufgaben» und «diskursiven Praktiken oder sprachlichen Verfahren zu deren Lösung» konstitutiv (ebd., S. 213).

Der Zusammenhang von «basaler Aktivität» einerseits und «kommunikativer Aufgabe» respektive «diskursiver Praktik» andererseits wird hier am Beispiel der basalen Aktivität «Ko-Konstruieren der Veränderung» verdeutlicht. Diese von Graf als «interaktives und thematisches Kernstück von Coaching» (ebd., S. 214) bezeichnete Aktivität enthält folgende «kommunikative Aufgaben»:

- «Diagnostizieren» (= Anliegensklärung),

- «Intervenieren» (= Anliegensbearbeitung),

- «Transfer sichern».

«Diagnostizieren» etwa besteht darin, die Ausrichtung eines Coachings, also das Anliegen, die Ziele und die angestrebten Veränderungen, diskursiv zu etablieren. Das «Intervenieren» zielt auf das sprachliche Generieren von Lösungen ab. Die dritte kommunikative Aufgabe, «Transfer sichern», besteht darin, erstens Erkenntnisse der Kundinnen, die während eines Coachings entstehen, auf deren professionellen Kontext zu übertragen, und zweitens, Erkenntnisse, die diese zwischen den Sitzungen gewinnen, in den Coaching-Prozess zu integrieren und damit auch den Coach auf den aktuellen Stand zu bringen (Graf 2015b, S. 214).

Die «diskursiven Praktiken» zur Bewältigung der drei «kommunikativen Aufgaben» werden in Anlehnung an die Habilitationsschrift von Graf (2015a, S. 535) auf Englisch aufgeführt, da sie in übersetzter Form nicht vorliegen. $\mathrm{Zu}$ den «diskursiven Praktiken» des «Diagnostizierens» gehören:

- «Eliciting and Presenting the Concern»,

- «Exploring and Defining the Concern»,

- «Mapping the Current State».

Die «diskursiven Praktiken» des «Intervenierens» sind:

- «Practicing Mindfulness»,

- «Processing the Concern on the Intra-Personal Level»,

- «Processing the Concern on the Inter-Personal Level»,

- «Linking the Intra-Personal and Inter-Personal Level». 
Die «diskursiven Praktiken» des «Transfer-Sicherns» sind abschließend:

- «Developing Transfer Practices»,

- «Corroborating Learning and Development».

In seiner Analyse der Arbeiten von Graf weist Geißler (2016, S. 56) präzisierend darauf hin, dass die «diskursiven Praktiken» mehrheitlich als basale Einzelhandlungen von Coach und Coachee zu verstehen sind und im Unterschied zu seinem Interpretationssystem nicht in weitere Einzelentscheidungen aufgeschlüsselt werden.

Graf (2015b) verwendet die «basalen kommunikativen Aktivitäten» und «Aufgaben» in Kombination mit sehr detaillierten Diskursanalysen auch zur Evaluation von Coaching-Prozessen. Dabei geht es um die zentrale Frage, wie die beschriebenen kommunikativen Prozesse zur Lösung der kommunikativen Aufgaben führen. Das zentrale Beurteilungskriterium stellen entsprechend die kommunikativen Reaktionen der Beteiligten dar, wie Graf in der folgenden Passage (ebd., S. 213) ausführt:

«Im Rahmen der qualitativen Herangehensweise linguistischer Forschung wird Schritt für Schritt aufgezeigt, wie die Beteiligten das Gespräch mit und in ihren Redebeiträgen konstruieren, und ihre Missverständnisse, Reparaturen, dispräferierten Antworten etc. werden als Indikatoren für Schwierigkeiten im Gespräch, d.h. Schwierigkeiten, die anfallenden kommunikativen Aufgaben zu lösen, interpretiert. Da sich Gespräche aus vielen einzelnen Redebeiträgen zusammensetzen, wird die Bedeutung einzelner Missverständnisse etc. jeweils im Kontext des gesamten Gesprächsverlaufs interpretiert. Die Qualität eines Gesprächs wird definiert als mehr oder weniger erfolgreiches Lösen der für einen bestimmten Gesprächstyp konstituierenden kommunikativen Aufgaben durch die Beteiligten, die sprachlich-kommunikativ gemäß ihren jeweiligen Rollen mit unterschiedlichen Verantwortlichkeiten und Beteiligungsvoraussetzungen agieren.»

Zur Veranschaulichung konkreter Evaluationensergebnisse wird hier eine Beschreibung angeführt, in der auch der Kommunikationsstil des Coaches und dessen Effekt auf die Coachee deutlich wird (ebd., S. 226f.):

«Gerade zu Beginn eines professionellen Gesprächs werden daher die Weichen gestellt für den weiteren Verlauf und das erfolgreiche Bearbeiten der kommunikativen Aufgaben. Und hier zeigt sich im vorliegenden Fall auch schon das vorherrschende interaktive Muster der Ko-Konstruktion der Veränderung: Der Coach geht auf der thematischen Ebene durch das Relevant-Setzen und Weiter- 
bearbeiten ausgewählter Aspekte der Darstellung der Klientin, durch das Übergehen thematischer Aspekte der Klientin, aber auch durch das Einbringen eigener Themen stark direktiv vor und behält diese thematische Dominanz entlang des gesamten Prozesses. Ein sprachliches Mittel dabei sind u.a. geschlossene Fragen und mangelnder Rederaum für die Klientin im Anschluss an diese Fragen. [...] In der letzten Sitzung wird noch einmal interaktiv deutlich, dass die Klientin entlang des Prozesses zwar der thematischen Entwicklung durch den Coach (vordergründig) folgt und ihrem Ziel so theoretisch näher kommt, ihre Zweifel und Bedenken aber auch am Ende des gesamten Prozesses nicht wirklich ausgeräumt sind. Dies legt die Frage nahe, inwieweit die Klientin die erarbeiteten Schritte zur Zielerreichung nach Beendigung des Coachings wirklich und nachhaltig umsetzen wird.»

Besonders zu würdigen am Vorgehen von Graf ist, dass, wie von ihr selbst hervorgehoben, dem dynamischen und komplexen Aspekt des Verlaufs von Coaching-Prozessen Rechnung getragen wird und als Beurteilungskriterium für die Bewertung der Qualität eines Coachings neben dem Coach-Verhalten vor allem auch die Reaktionsweisen der Coachees mitberücksichtigt werden.

\section{Das Kategoriensystem KaSyCo von Silvia Deplazes}

Deplazes entwickelt in ihrer Promotionsarbeit (Deplazes 2016) ein Kategoriensystem zur Analyse von Coaching-Prozessen (KaSyCo), mittels dessen «Interventionen» und «Tools» der Coach- und Coachee-Aktivitäten erfasst werden. Anhand von 27 videoaufgezeichneten Coaching-Sitzungen wurden induktiv Kategorien gebildet. Die methodische Grundlage dafür bildete eine strukturierende qualitative Inhaltsanalyse (ebd., S. VII).

Das System umfasst insgesamt 85 Kategorien, mittels deren Coach-Interventionen erfasst werden. Diese verortet Deplazes in einer Matrix von acht «Interventionsformen» und fünf «inhaltlichen Fokussen». Die Interventionsformen sind «Zuhören», «Reflexion», «Fragen», «Handlungsanleitung», «Erläuterung», «Stellungnahme», «Humor» und «übrige». Die inhaltlichen Fokusse sind «Sachebene/Klientensystem», «Erleben und Verhalten der Klienten», «Körperwahrnehmung und körperlicher Ausdruck», «Coaching-Prozess» und «andere».

Als exemplarische Darstellung werden in der folgenden Übersicht die in der Analyse identifizierten Fragetypen (= eine der acht Coach-Interventionen) und darauf bezogene inhaltliche Fokusse präsentiert (vgl. Tabelle 2.12).

In Anlehnung an das sprechakttheoretische Fundament des «sinnerschließenden Interpretationssystems» von Geißler (2016) lässt sich hier erkennen, dass Deplazes zur Bestimmung der 85 Kategorien - hier illustriert am Beispiel der Interventionsform «Fragen»- mit «Interventionsformen» einen illokutionären Fokus setzt, mittels dessen die Beziehung von Coach, Coachee und Gesprächsinhalt be- 
stimmt wird. Die (hier fünf) «inhaltlichen Fokusse» präzisieren im Sinne eines propositionalen Aspekts den Kommunikationsinhalt der Gesprächspartner. Entsprechend weisen die Systeme von Deplazes und Geißler eine gewisse Verwandtschaft auf.

Tabelle 2.12 Induktiv identifizierte Fragetypen zur Erfassung von Coach-Interventionen nach Deplazes (2016, S. 242)

\begin{tabular}{|c|c|c|c|c|c|}
\hline \multicolumn{6}{|l|}{ Fragetypen } \\
\hline & zirkuläre Frage & $\begin{array}{l}\text { hypothetische } \\
\text { Frage }\end{array}$ & $\begin{array}{l}\text { Skalierungs- } \\
\text { frage }\end{array}$ & $\begin{array}{l}\text { offene } \\
\text { Frage }\end{array}$ & $\begin{array}{l}\text { geschlossene } \\
\text { Frage }\end{array}$ \\
\hline \multirow{3}{*}{$\begin{array}{l}\text { Sachebene/ } \\
\text { Klientensystem }\end{array}$} & Frage zum & Frage zum & Frage zum & Frage zum & Frage zum \\
\hline & Klientensystem & Klientensystem & Klientensystem & Klientensystem & Klientensystem \\
\hline & Einstiegsfrage & Einstiegsfrage & Einstiegsfrage & Einstiegsfrage & Einstiegsfrage \\
\hline \multirow[t]{7}{*}{$\begin{array}{l}\text { Erleben und } \\
\text { Verhalten des } \\
\text { Klienten }\end{array}$} & $\begin{array}{l}\text { Fragen nach } \\
\text { dem Erleben } \\
\text { und Verhalten }\end{array}$ & $\begin{array}{l}\text { Fragen nach } \\
\text { dem Erleben } \\
\text { und Verhalten }\end{array}$ & $\begin{array}{l}\text { Fragen nach } \\
\text { dem Erleben } \\
\text { und Verhalten }\end{array}$ & $\begin{array}{l}\text { Fragen nach } \\
\text { dem Erleben } \\
\text { und Verhalten }\end{array}$ & $\begin{array}{l}\text { Fragen nach } \\
\text { dem Erleben } \\
\text { und Verhalten }\end{array}$ \\
\hline & Frage nach den & Frage nach den & Frage nach den & Frage nach den & Frage nach den \\
\hline & Bedürfnissen & Bedürfnissen & Bedürfnissen & Bedürfnissen & Bedürfnissen \\
\hline & Frage nach & Frage nach & Frage nach & Frage nach & Frage nach \\
\hline & Bildern & Bildern & Bildern & Bildern & Bildern \\
\hline & Frage zum & Frage zum & Frage zum & Frage zum & Frage zum \\
\hline & Transfer & Transfer & Transfer & Transfer & Transfer \\
\hline \multirow{4}{*}{$\begin{array}{l}\text { Körperwahr- } \\
\text { nehmung, körper- } \\
\text { licher Ausdruck }\end{array}$} & Frage nach & Frage nach & Frage nach & Frage nach & Frage nach \\
\hline & $\begin{array}{l}\text { Körperwahr- } \\
\text { nehmung oder } \\
\text { körperlichem } \\
\text { Ausdruck }\end{array}$ & $\begin{array}{l}\text { Körperwahr- } \\
\text { nehmung oder } \\
\text { körperlichem } \\
\text { Ausdruck }\end{array}$ & $\begin{array}{l}\text { Körperwahr- } \\
\text { nehmung oder } \\
\text { körperlichem } \\
\text { Ausdruck }\end{array}$ & $\begin{array}{l}\text { Körperwahr- } \\
\text { nehmung oder } \\
\text { körperlichem } \\
\text { Ausdruck }\end{array}$ & $\begin{array}{l}\text { Körperwahr- } \\
\text { nehmung oder } \\
\text { körperlichem } \\
\text { Ausdruck }\end{array}$ \\
\hline & $\begin{array}{l}\text { Frage nach } \\
\text { psychodrama- } \\
\text { tischen }\end{array}$ & $\begin{array}{l}\text { Frage nach } \\
\text { psychodrama- } \\
\text { tischen }\end{array}$ & $\begin{array}{l}\text { Frage nach } \\
\text { psycho- } \\
\text { dramatische }\end{array}$ & $\begin{array}{l}\text { Frage nach } \\
\text { psycho- } \\
\text { dramatischen }\end{array}$ & $\begin{array}{l}\text { Frage nach } \\
\text { psycho- } \\
\text { dramatischen }\end{array}$ \\
\hline & Elementen & Elementen & Elementen & Elementen & Elementen \\
\hline \multirow[t]{5}{*}{$\begin{array}{l}\text { Coaching-Pro- } \\
\text { zess }\end{array}$} & $\begin{array}{l}\text { Zielklärungs- } \\
\text { frage }\end{array}$ & $\begin{array}{l}\text { Zielklärungs- } \\
\text { frage }\end{array}$ & $\begin{array}{l}\text { Zielklärungs- } \\
\text { frage }\end{array}$ & $\begin{array}{l}\text { Zielklärungs- } \\
\text { frage }\end{array}$ & $\begin{array}{l}\text { Zielklärungs- } \\
\text { frage }\end{array}$ \\
\hline & Frage zum & Frage zum & Frage zum & Frage zum & Frage zum \\
\hline & Verlauf des & Verlauf des & Verlauf des & Verlauf des & Verlauf des \\
\hline & $\begin{array}{l}\text { Coaching-Pro- } \\
\text { zesses }\end{array}$ & $\begin{array}{l}\text { Coaching-Pro- } \\
\text { zesses }\end{array}$ & $\begin{array}{l}\text { Coaching-Pro- } \\
\text { zesses }\end{array}$ & $\begin{array}{l}\text { Coaching-Pro- } \\
\text { zesses }\end{array}$ & $\begin{array}{l}\text { Coaching-Pro- } \\
\text { zesses }\end{array}$ \\
\hline & $\begin{array}{l}\text { Ziel- } \\
\text { erreichungs- } \\
\text { frage }\end{array}$ & $\begin{array}{l}\text { Ziel- } \\
\text { erreichungs- } \\
\text { frage }\end{array}$ & $\begin{array}{l}\text { Ziel- } \\
\text { erreichungs- } \\
\text { frage }\end{array}$ & $\begin{array}{l}\text { Ziel- } \\
\text { erreichungs- } \\
\text { frage }\end{array}$ & $\begin{array}{l}\text { Ziel- } \\
\text { erreichungs- } \\
\text { frage }\end{array}$ \\
\hline
\end{tabular}

Zur Kategorisierung der Coach-Interventionen identifiziert Deplazes ferner acht «Tools», die nicht weiter strukturiert wurden (Deplazes 2016, S. 138 f.). Diese sind 
«imaginativer Rollentausch», «Arbeit mit inneren Anteilen», «Accessing», «Embodiment», «Visualisierung der gemeinsamen Arbeit», «Visualisierung fachlicher Inputs», «Rollenspiel des Coaches» und «Reflecting Team».

Das System umfasst weiter 26 Kategorien zur Erfassung der Coachee-Aktivitäten, eingebettet in die Achsen «Aktivitätsformen» und «inhaltliche Fokusse» (ebd., S. 141 ff.). Damit wird auch für die Verortung dieser Kategorien zwischen einer illokutionären und einer propositionalen Dimension unterschieden. Die neun Aktivitätsformen sind «Erläuterung», «Reflexion», «Antizipation», «Imagination», «Self-Awareness», «Physische Tätigkeit», «Zuhören», «Humor» und «übrige». Die fünf «inhaltlichen Fokusse» sind «Sachebene/Klientensystem», «Erleben und Verhalten», «Körperwahrnehmung und -ausdruck», «Coaching-Prozess» und «andere».

Da das Kategoriensystem zur Erfassung der Coachee-Aktivitäten weniger umfangreich ist, wird es hier (vgl. Tabelle 2.13) vollständig abgebildet (ebd., S. 287).

Auf der Grundlage statistischer Analysen konnte Deplazes durch die Anwendung des Kategoriensystems zeigen, dass professionelle Coaches (ebd., S. 175) vor allem «zuhören», «Fragen stellen», «Aussagen der Kundinnen reflektieren», «Ratschläge formulieren» und «eigene Meinungen äußern». Das Verhalten der Coachees ist dagegen mehrheitlich bestimmt durch «zuhören», «reflektieren» und «das eigene Umfeld erläutern».

In weiteren Untersuchungen konnte Deplazes zudem zeigen, dass die ambivalente Lehrmeinung zum Einsatz direktiver und non-direktiver Interventionen sich in der Coaching-Praxis nicht wiederfinden lässt, da in beinahe allen von ihr untersuchten Coaching-Sitzungen direktive Interventionen beobachtet wurden (ebd.). In einer weiteren Untersuchung konnte Deplazes darüber hinaus zeigen, dass sich das Antwortverhalten der Coachees bei offenen und geschlossenen Frage kaum unterscheidet (ebd.).

Besonders zu würdigen am Ansatz von Deplazes ist, dass sie ein umfassendes Kategoriensystem zur Bestimmung der Coach- und Coachee-Aktivitäten gebildet hat, dass sowohl illokutionäre als auch propositionale Dimensionen aufweist. Darüber hinaus sind auch die weiterführenden Anwendungen des Kategoriensystems, wie eben beschrieben, als positiv zu bewerten. Es bleibt zu hoffen, dass weitere Fragestellungen, in denen vor allem auch der Zusammenhang von Coach- und Coachee-Verhalten stehen, mittels des Kategoriensystems und im Sinne des «Microanalytic Sequential Process Design» untersucht werden. 


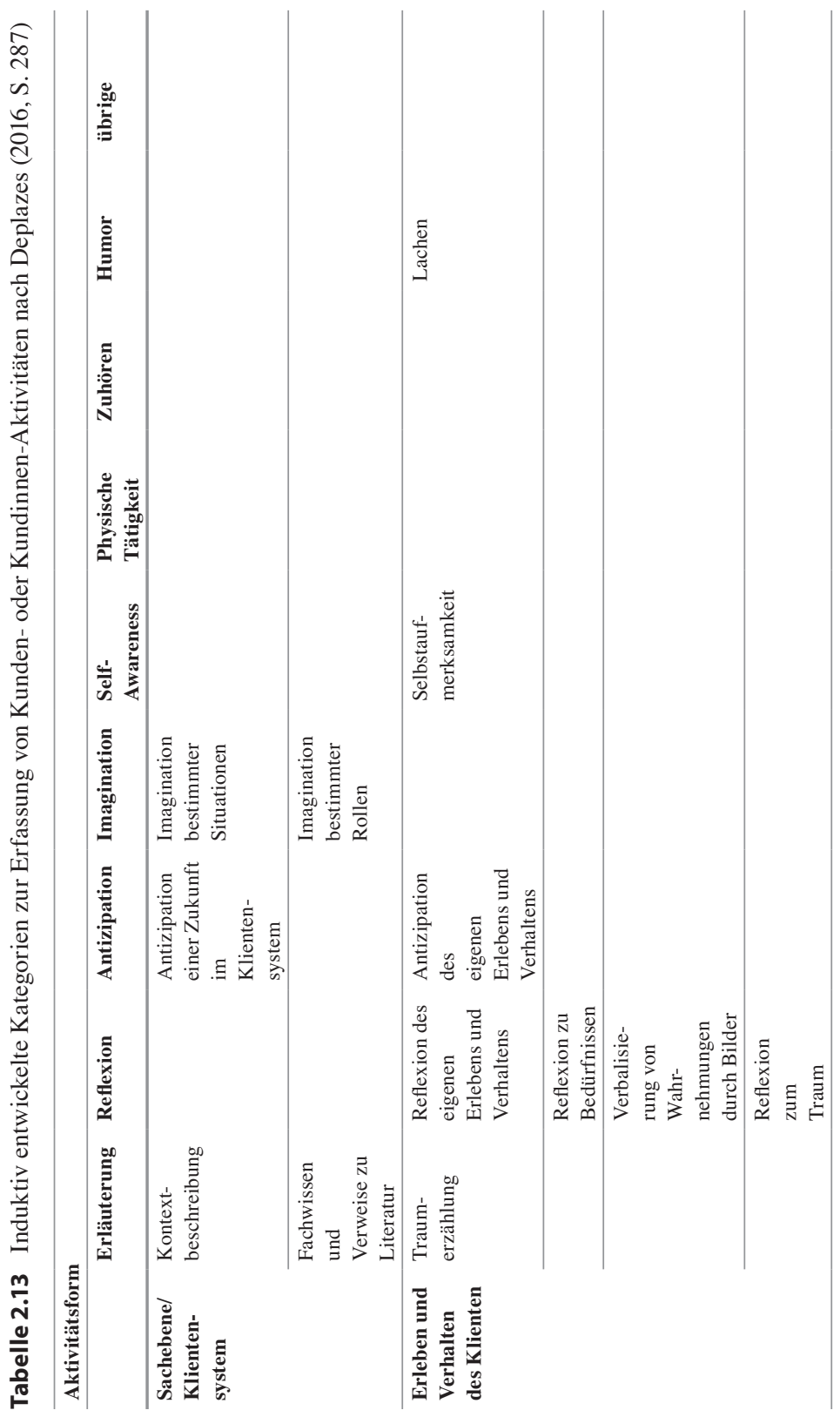




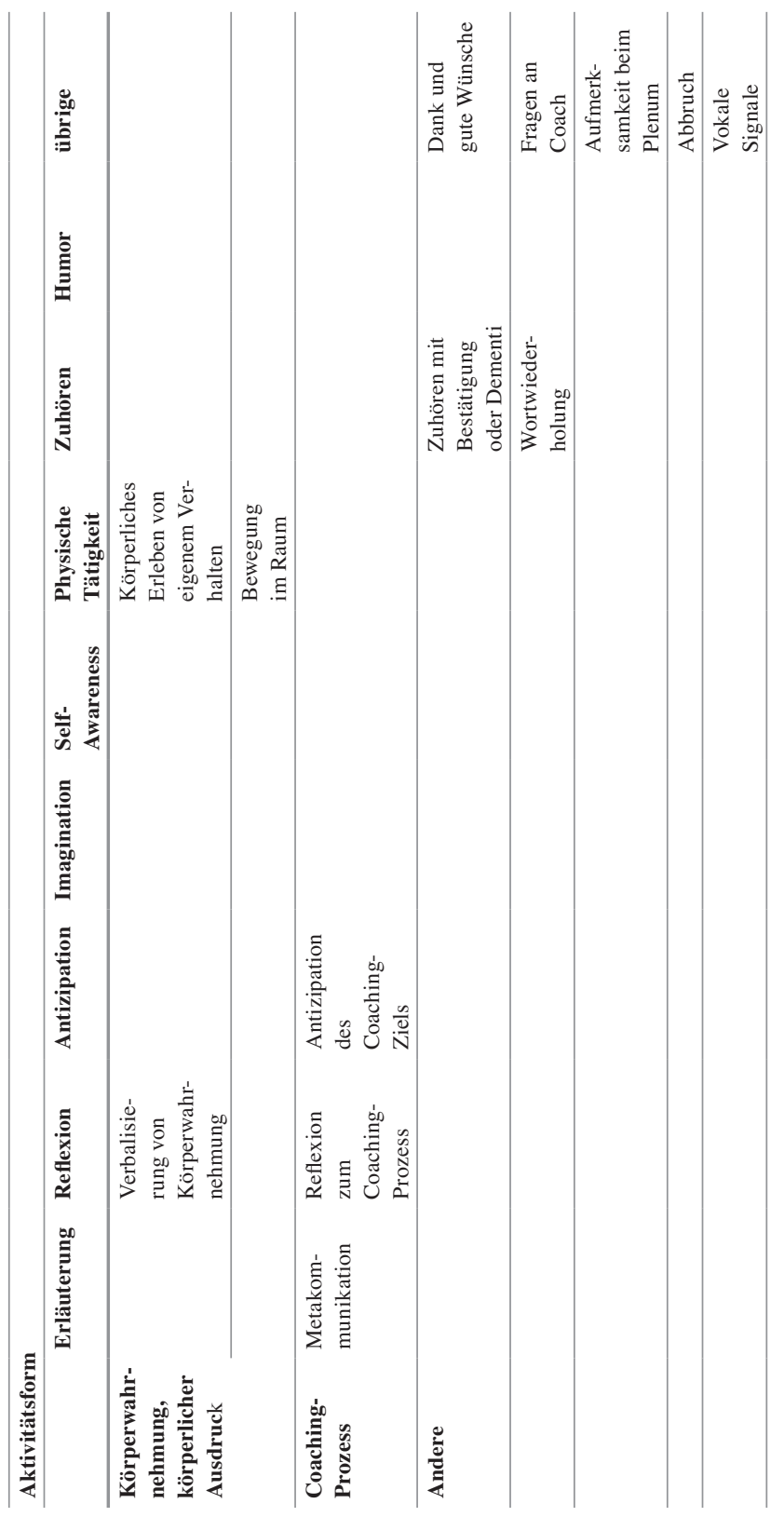




\section{Diskussion der vorgestellten Studien}

In den vorangehend präsentierten Forschungsansätzen geht es darum, zu untersuchen, worin professionelle Coachings als Verhaltensweisen respektive Interaktionssequenzen von Coach und Coachee konkret bestehen. In allen Ansätzen wurden zu diesem Zweck Kategorien entwickelt und mehrheitlich überprüft, wie die einzelnen Ansätze Coaching-Verläufe besonders gut beschreiben.

So kommt Geißler etwa zum Schluss, dass Coaches oft Fragen stellen und das Gesagte der Coachees spiegeln. Damit wählen Coaches mehrheitlich facilitative Sprechhandlungen. Coachees dagegen vermitteln oft fallspezifisches Wissen und offenbaren sich häufig selbst, womit sie oft instruktionalistischen Sprechhandlungen den Vorrang geben.

Das Team von Tatiana Bachkirova kommt zu einem ähnlichen Schluss, jedoch nicht aus einer Auswertung von Coaching-Verläufen, sondern aufgrund erfasster Lehrmeinungen professioneller Coaches, orientiert am von Bachkirova et al. entwickelten «Descriptor»-Set. So wird in ihrer Untersuchung deutlich, dass Coaching durch zwei Fragetypen besonders gut charakterisiert werden kann:

- «Coach asks questions helping the client to elaborate.»

- «Coach asks questions that appear to open new possibilities for the client.»

Zudem lässt sich gemäß den Untersuchungen von Bachkirova et al. Coaching durch folgende Deskriptoren gut von anderen Berartungsformaten abgrenzen:

- 7 «Coach and client explore the deeper meaning of a presenting issue.»

- 8 «Coach points out a recurrent theme in client's behavior.»

Deplazes generiert durch ihre Analysen die Erkenntnis, das Coaches vor allem «zuhören», «Fragen stellen», «Aussagen der Kundinnen reflektieren», «Ratschläge formulieren» und «eigene Meinungen äußern». Coachees zeigen vor allem folgende Verhaltensweisen: «zuhören», «reflektieren» und «das eigene Umfeld erläutern». Mit diesen Ergebnissen werden jene von Geißler und Bachkirova gewisserweise infrage gestellt - ein Befund, der in weiteren Untersuchungen überprüft und entsprechend erklärt werden muss.

Entsprechende Häufigkeitsauszählungen liegen für die Kategorien von Graf bis anhin nicht vor, weshalb ein entsprechender Ergebnisvergleich nicht möglich ist.

Der Ansatz von Geißler fällt durch seine außerordentlich präzise, auf elf Entscheidungsdimensionen basierende Bestimmung einzelner Kommunikationsentscheidungen als wichtige Unterscheidungsmerkmale auf. Angesichts der - aus kombinatorischen Gründen betrachtet - enormen Vielfalt an Kommunikations- 
entscheidungsvarianten kann die anvisierte «Entscheidungssystematik» noch nicht als endgültig bezeichnet werden, sondern als Work in Progress. Ein konkretes Beispiel für diesen Rekonstruktionsprozess ist die umfassende Fragentypologie, die Geißler in Zusammenhang mit den Entscheidungsdimensionen entwickelt hat (vgl. Geißler 2016, S. 375-464)

Das «Descriptor»-Set von Bachkirova et al. wurde genutzt, um Lehrmeinungen professioneller Coaches mit Blick auf charakteristische Merkmale von Coaching zu erfassen und Coaching damit von anderen Beratungsformen auch abgrenzen zu können. Die Untersuchung faktischer Coaching-Prozesse steht noch an. Dazu sollen aus den identifizierten «Descriptors» zunächst noch Skalen entwickelt werden.

Der linguistische Ansatz von Graf charakterisiert sich über das drei Abstraktionsstufen umfassende Kategoriensystem, in dem «basale kommunikative Aktivitäten» von «kommunikativen Aufgaben» und «diskursiven Praktiken» unterschieden werden. Bezeichnend für den Ansatz ist ferner der Fokus auf das interaktive Geschehen zwischen Coach und Coachee (Ko-Konstruktionsprozess) respektive die Annahme, dass es im Kern von Coaching darum geht, kommunikative Aufgaben erfolgreich zu bewältigen. Besonders wertvoll erscheint dabei der Versuch, dem komplexen und dynamischen Charakter von Coaching durch die Abbildung (wiederkehrender) «basaler kommunikativer Aktivitäten» gerecht zu werden. Weiter ist auch die Evaluation der Qualität von Coaching-Verläufen über das Kriterium der unmittelbaren Coachee-Reaktion als positiv zu werten, da damit die wichtige Position der Coachees gebührende Beachtung erhält.

Der Ansatz von Deplazes verwendet zur Beschreibung der identifizierten Kategorien - analog zu Geißler - zwei sprechakttheoretische Dimensionen: die Dimension der Interventions-/Aktivitäten-Form (= illokutionärer Aspekt) und die Dimension der inhaltlichen Fokusse (= propositionaler Aspekt). Durch diese zweifache Bestimmung der Kategorien weist das System eine Nähe zum Interpretationssystem von Geißler auf und hebt sich zugleich von den Ansätzen von Bachkirova und Graf ab.

\subsubsection{Präzisierung des Forschungsdesiderats}

Mit diesem Streifzug durch die Landschaft an Studien und Forschungansätzen, die der oben skizzierten Coaching-Forschungslandkarte (vgl. Kapitel 2.3.1) zugeordnet werden können, erfolgt nun - im Anschluss an der an Greifs Evaluationsmodell orientierten Darstellung - eine zweite Bilanzierung des Forschungsstands. 


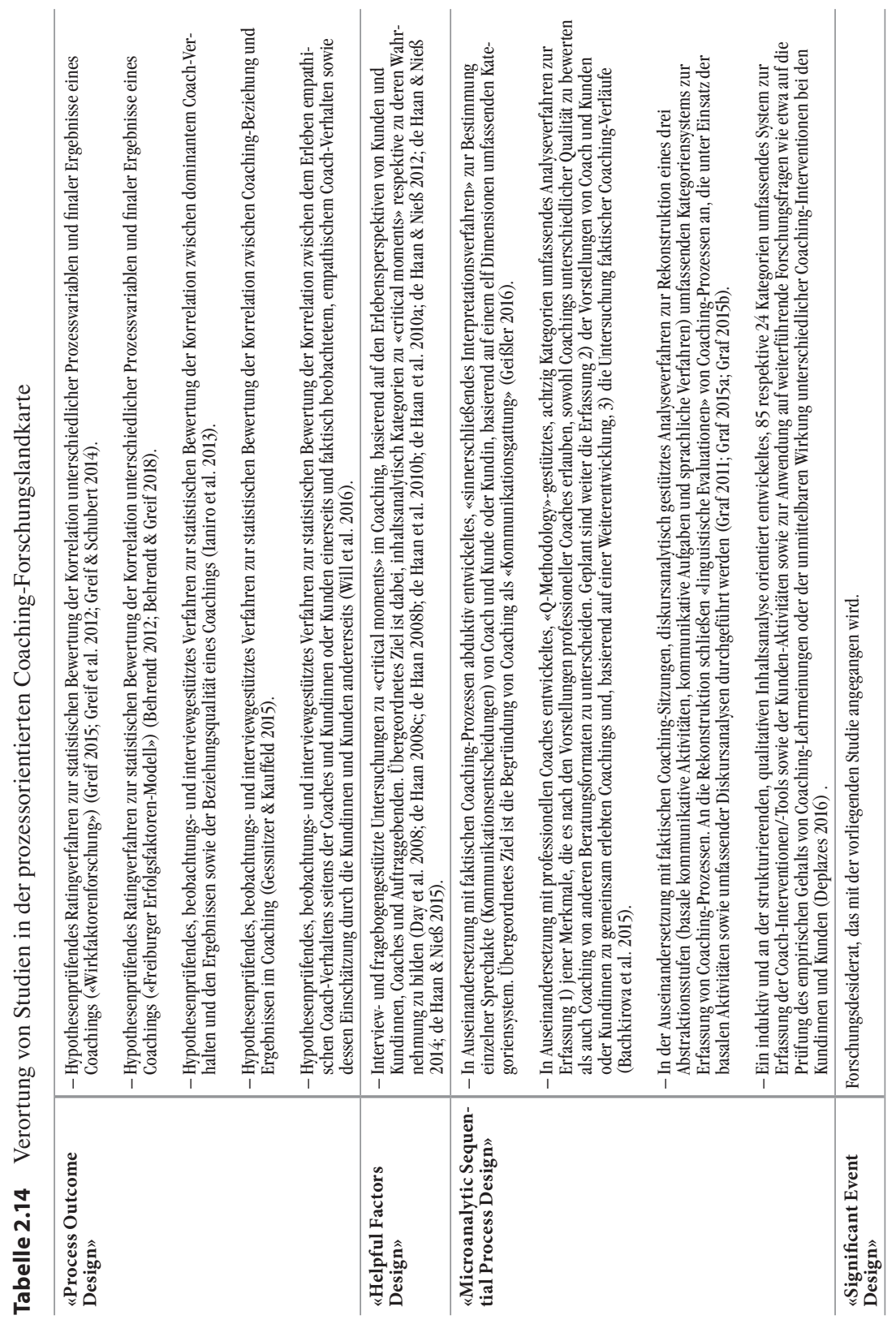


Dabei wird deutlich, dass die Forschungslandkarte wichtige Forschungsthemen und -zugänge adressiert und dass Untersuchungen dazu auch schon existieren. Die Bilanzierung macht aber auch deutlich (vgl. dazu auch Tabelle 2.14), dass noch viele relevante Forschungsfragen unbeantwortet bleiben und insbesondere auch noch keine «Significant Event Design»-orientierte Studien vorliegen. In solchen Studien ginge es darum, bedeutsame Coaching-Ereignisse auf der Grundlage faktischer Coaching-Verläufe zu erforschen.

Diesem Forschungsanliegen ist die vorliegende Arbeit gewidmet. Der Grund liegt darin, dass ein solcher Forschungszugang dem komplexen und dynamischen Charakter von Coaching in besonderer Weise gerecht werden kann. Im Sinne der Orientierungsfunktion von Coaching-Forschung (Fietze 2011) soll damit ein Beitrag an die weitere Professionalisierung von Coaching geleistet werden. Die Intention ist also, weiter «Licht in die Blackbox des Beratungsprozesses [zu] bringen und vor allem diejenigen Aspekte [zu] beleuchten, die durch professionelles Handeln beeinflusst werden und durch empirische Erkenntnisse optimiert werden können» (ebd., S. 28). Praktikerinnen und Praktikern soll so im besten Fall eine Handlungsorientierung respektive eine «Entscheidungshilfe in Zweifelsfragen, eine Risikominderung ihrer professionellen Interventionen und eine Entlastung ihrer Handlungsverantwortung» (ebd., S. 29) an die Hand gegeben werden. Dabei wird die Handlungsorientierung aufgrund des Bezugs zur zentral steuerungsrelevanten Idee «bedeutsamer Ereignisse» idealerweise hoch sein.

\section{4 «Significant Event Design»-orientierte Psycho- therapie-Studien als Ausgangspunkt für das Design zur Untersuchung «bedeutsamer Momente» im Coaching}

Als Ausgangspunkt für die Bestimmung des Forschungsdesigns der Untersuchung $\mathrm{zu}$ «bedeutsamen Momenten» im Coaching werden drei psychotherapieorientierte Forschungsansätze vorgestellt: die «Task Analysis» (Greenberg 1984; Greenberg 2007; Pascual-Leone, Greenberg \& Pascual-Leone 2009), die «Comprehensive Process Analysis» (Elliott et al. 1994) und die «Assimilation Analysis» (Stiles et al. 1990). Elliott ordnet diese drei Ansätze dem «Significant Event Design» des «Change Process Research»-Paradigmas zu, da in allen relevante Therapieereignisse auf der Grundlage faktischer Therapieverläufe untersucht werden.

Die zentralen methodologischen, theoretisch-inhaltlichen und methodischen Aspekte dieser drei Ansätze werden nun als Grundlage des anschließend in Kapitel 3 präsentierten Forschungsdesigns vorgestellt. 


\subsection{1 «Task Analysis»}

\section{Ziel}

In der «Task Analysis» wird geprüft, wie Patientinnen und Patienten ihre psychologischen Probleme, gerahmt als «therapeutische Aufgaben» («Tasks»), erfolgreich bearbeiten. Als Forschungsoutput werden Modelle entwickelt, in denen jene Teilaufgaben enthalten sind, die zur Bewältigung der jeweiligen therapeutischen Aufgabe bearbeitet werden müssen. Beispiele solcher Aufgaben sind das Überwinden von Unentschiedenheit oder Hilflosigkeit sowie das Lösen von Beziehungsproblemen (Greenberg 2007, S. 17).

Der Ansatz wird den «Significant Event Design»-orientierten Psychotherapie-Studien zugeordnet, weil es dem Begründer Greenberg darum ging, «to study the performance of the client changes in these key moments», wobei ein «moment» als «clinically meaningful client-therapist interactional sequence« definiert wird (ebd., S. 15 f.).

\section{Ursprung}

Der Ursprung der «Task Analysis» liegt in Management-Studien zum Verlauf von Arbeitsprozessen. Diese wurden in den 1940er Jahren durchgeführt. In den 1980er Jahren übertrug Greenberg die Idee der Strukturierung von Arbeitsprozessen auf die Analyse psychotherapeutischer Veränderungsprozesse (ebd., S. 15 f.).

\section{Analyseschritte}

Die «Task Analysis», die von therapeutisch geschulten Wissenschaftlern und Wissenschaftlerinnen durchgeführt werden soll, umfasst sowohl eine Entdeckungs- als auch eine Validierungsphase (ebd., S. 16). Zu Beginn der Entdeckungsphase wird das vorliegende und zu untersuchende, «affektiv-kognitive Problem» im Sinne der therapeutischen Aufgabe spezifiziert («specifying the taks»). Dazu werden Verhaltensmarker («behavioral marker»), in denen der Problemzustand besonders deutlich erkennbar wird, im Datenmaterial identifiziert, und das zu untersuchende Problem wird beschrieben. Etwa so: «The presence of a lingering, unresolved feeling such as hurt or resentment» (ebd., S. 17).

Als Nächstes werden in Form theoretischer Annahmen Teilaufgaben und damit verbundene Arbeitsprozesse beschrieben, die zur Lösung des Problems notwendig erscheinen (ebd.). Mit Bezug darauf wird nun ein «theoretisches Modell» (für ein konkretes Beispiel vgl. Abbildung 2.4) entwickelt («construct the rational model»). Fragen, die sich die auch praktisch-therapeutisch tätigen Forschenden während dieses Schrits stellen, sind etwa: «How do I think clients resolve this particular 
task?» oder «What essential performance steps must the client go through to resolve this problem?» (ebd., S. 19).

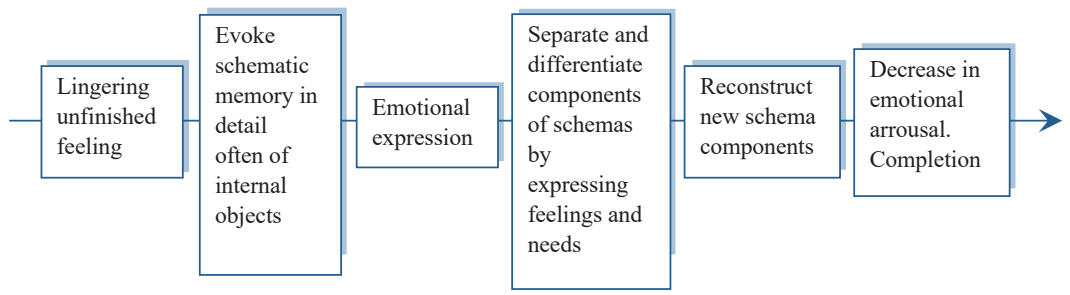

Abbildung 2.4 Theoretisches Modell zur Überwindung ungelöster Gefühle («unfinished business») (Quelle: Greenberg 2007, S. 19)

Wie das folgende Zitat verdeutlicht, liegt der Sinn dieses theoretischen Modells darin, einen möglichst neutralen Feldzugang sicherzustellen:

«As we have said, the investigators, as best they can, attempt to bracket their preconceptions and suspend their anticipations, hunches, and expectations, made explicit in the rational model, to receive, in as uninvested a fashion as possible, what is there to observe in the actual performance.» (Greenberg 2007, S. 19)

Nach der Entwicklung des theoretischen Modells wird das Fallmaterial auf darin nachweisbare Teilaufgaben untersucht («conduct the empirical task analysis»). In Anlehnung an die Grounded Theory wird nun, basierend auf vergleichbaren Fällen, ein «empirisches Modell» der zur Lösung der therapeutischen Aufgabe notwendigen Teilaufgaben entwickelt (für Details dazu vgl. ebd., S. 19-22). Ebenso gilt es in diesem Schritt, Messverfahren zu entwickeln, mit denen die Bewältigung der identifizierten Teilaufgaben in der noch bevorstehenden Validierungsphase überprüft werden können (ebd., S. 22).

Im letzten Schritt der Entdeckungsphase werden das theoretische und das empirische Modell in ein theoretisch-empirisches Modell («synthesizing a rational-empirical model») übergeführt (als Beispiel vgl. Abbildung 2.5). Dabei geht es darum, das theoretische Modell im Kontrast zum empirischen Modell zu bestätigen, zu erweitern oder zu modifizieren (ebd., S. 23).

Greenberg präzisiert, dass es sich bei diesem Modell nicht um ein lineares Ablauf-, sondern um ein Strukturmodell handelt, indem die notwendigen Teilaufgaben und damit verbundenen Arbeitsprozesse abgebildet werden. Feedbackprozesse, Rückwärts- oder Vorwärtsbewegungen der Patientinnen und Patienten werden nicht berücksichtigt (ebd.). 


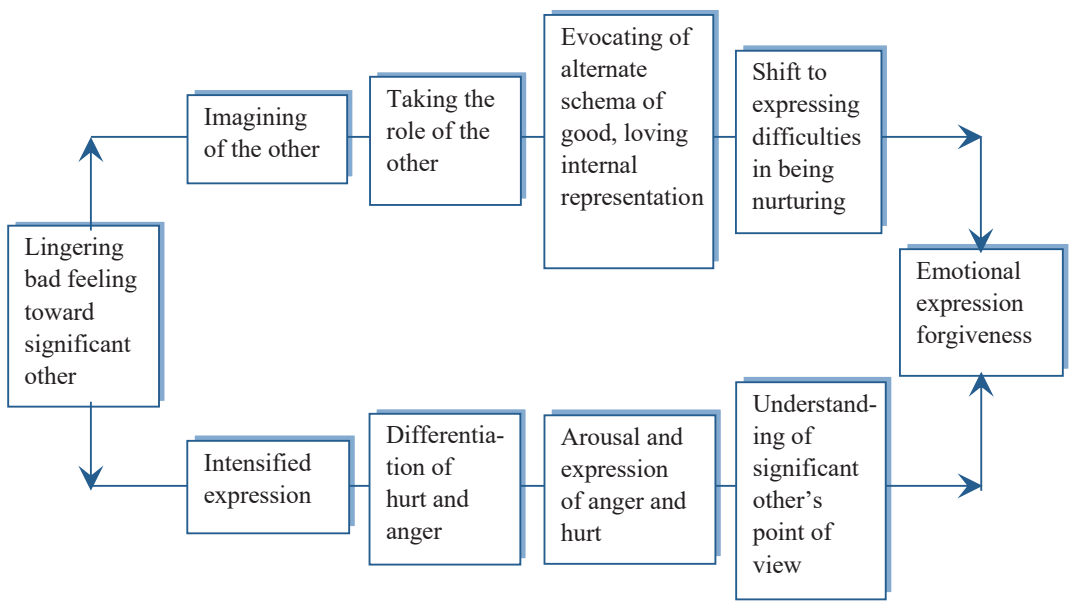

Abbildung 2.5 Theoretisch-empirisches Modell der Überwindung einer unvollständigen Erfahrung («incomplete experience») (Quelle: Greenberg 2007, S. 23)

In der hier nicht ausgeführten Validierungsphase wird das theoretisch-empirische Modell im Sinne eines deduktiven Vorgehens empirischen Prüfungen unterzogen (für weitere Details vgl. ebd., S. 26 ff.).

\section{Methodologische und erkenntnistheoretische Grundlagen}

Methodologische Grundlage der Entdeckungsphase der «Task Analysis» ist das Modell der Abduktion nach Peirce (Harthshorne, Weiss \& Burks 1965). Kerngedanke dabei ist, dass ein theoretisches Modell durch empirische Beobachtungen so lange weiterentwickelt wird, bis eine Sättigung auftritt (Greenberg 2007, S. 25).

Besonders an der «Task Analysis» ist, dass im Unterschied zu herkömmlichen Anwendungen der Grounded Theory oder der phänomenologischen Analyse nicht die subjektiven Perspektiven der Patientinnen und Patienten, sondern ihre faktischen Verhaltensweisen und ihr Sprechverhalten analysiert und ausgewertet werden (ebd.).

Greenberg (ebd., S. 26) rechnet den hier beschriebenen Weg der Entwicklung eines theoretisch-empirischen Modells, erkenntnistheoretisch gesprochen, dem «dialektisch-konstruktivistischen» Paradigma zu (Greenberg \& Pascual-Leone 1995), das sich zwischen Realismus und Konstruktivismus positioniert, also davon ausgeht, dass die Wirklichkeit bis zu einem gewissen Grad konstruiert ist, teilweise aber auch als solche erkannt werden kann. Dazu ein präzisierendes Zitat: 
"In this view, although it is not possible to be completely objective, and that observation is not theory free, reality is offering constrains from which we can construct what we know. Thus, although we can never know what is actually out there, it is possible to make progressive approximations toward describing constraints that do exist in reality. Investigation then results in the construction of explanatory models that mediate between empirical reality and social construction [...]. This is the goal of task analysis: to construct explanatory models that help to capture some of the invariants across resolution performance.»(Greenberg 2007, S. 26)

\subsection{2 «Comprehensive Process Analysis»}

\section{Ziel}

Die «Comprehensive Process Analysis», kurz CPA (Elliott 1989; Elliott et al. 1994), zählt deshalb zu den «Significant Event Design»-orientierten Psychotherapie-Studien, weil darin Merkmale, Einflussfaktoren und Effekte ausgewählter Klassen von «important moments of therapeutic change» untersucht und passende Modelle dazu entwickelt werden (Elliott et al. 1994, S. 180 ff.). Im Unterschied zur «Task Analysis» geht es somit nicht um die umfassende Analyse der Bearbeitung konkreter psychischer Probleme.

\section{Ursprung, Grundbegriffe und Grundannahmen}

Elliott positioniert die CPA als qualitative Forschungsstrategie, die sich im Unterschied zu quantitativen Untersuchungen durch weniger reduzierende Therapieannahmen auszeichnet. Im Kern der CPA und in der genannten Abgrenzung liegt die Annahme, dass nicht alle Ereignisse einer Therapie für den Veränderungsprozess von Patientinnen und Patienten gleich wichtig sind, sondern dass deren Wichtigkeit variiert (vgl. dazu Elliott 1989, S. 167 f.). Auf dieser Grundlage untersucht Elliott klinisch relevante Ereignisse, die er als «turning points» bezeichnet und in denen sich für Patientinnen und Patienten etwas «zum Besseren» wendet (Elliott 1984, S. 251).

Bedeutsame Therapieereignisse definiert er so:

«Significant therapy events consist of sequences of client and therapist actions which facilitate specific psychological impacts in clients. For example, if a client requests help in understanding something about the self and the therapist offers an interpretation involving the client's core conflicts, delivered in a warm, collaborative manner, the client may experience helpful insight into the self.» (Elliott 1989, S. 165) 
Elliott betont mit bedeutsamen Therapieereignissen somit das produktive $\mathrm{Zu}$ sammenspiel von Therapeutenintervention und Klientenprozessen, deren Existenz gemäß seinen Ausführungen bereits von anderen Wissenschaftlerinnen und Wissenschaftlern bestätigt wurde (z.B. Elliott et al. 1985; Kelman 1969; Mahrer \& Nadler 1986). Ein weiteres Merkmal der CPA ist, dass phänomenologische Daten im Sinne der Erlebensperspektive und auch Verhaltensdaten im Sinne faktischer Therapieverläufe zur Analyse bedeutsamer Ereignisse berücksichtigt werden (Elliott 1984).

\section{Sechs Analyseprinzipien}

Das methodische Vorgehen der CPA orientiert sich an sechs Analyseprinzipien. Das erste besagt, das je Untersuchung nur eine Klasse bedeutsamer Therapieereignisse untersucht wird. Grund dafür ist die Annahme, dass in Therapieprozessen enthaltene Ereignisse zu heterogen sind, um sie auf sinnvolle Weise miteinander verbinden zu können (Elliott 1989, S. 169). Das zweite Prinzip besagt, dass die identifizierten Therapieereignisse anhand des «Framework for Comprehensive Process Analysis» (vgl. Tabelle 2.15) analysiert werden. Dieses enthält «Faktoren», die helfen, bedeutsame Therapieereignisse zu verstehen.

Das dritte CPA-Prinzip besagt, dass drei bis fünf Forschende die ausgewählten Therapieereignisse auswerten sollen, um nicht Gefahr zu laufen, wichtige Aspekte zu übersehen. Im Unterschied zu Ratingverfahren, in denen in der Regel ebenfalls mehrere Forschende parallel arbeiten, werden die Forschenden in diesem qualitativen Ansatz nicht durch vorgängig gesetzte Kategorien oder Ratingskalen eingeschränkt. Stattdessen geht es darum, induktiv und im diskursiven Austausch zu entsprechenden Erkenntnissen zu gelangen. Idealerweise bringen die Forschenden - analog zur «Task Analysis» - klinische Erfahrung mit (ebd., S. 171 f.).

Das vierte CPA-Prinzip betont, dass auch quantitative Instrumente eingesetzt werden sollen - dies, um unabhängig von den beschriebenen qualitativen Analyseschritten die klinische Relevanz der untersuchten Ereignisse zu überprüfen (Elliott 1989, S. 172). Ein Beispiel für ein solches Instrument ist das «Patient Experiencing Rating» nach Klein, Mathieu-Coughlan und Kiesler (1986).

Das fünfte Prinzip der CPA beinhaltet, dass die Annahmen und Erwartungen der Forschenden, analog zur «Task Analysis», sichtbar gemacht werden sollen dies, um zu verhindern, dass nicht explizierte Annahmen die Untersuchungsergebnisse negativ beeinflussen. Als Ausdruck für dieses Prinzip schreibt Elliott «letting the data 〈speak for themselves»» (Elliott 1989, S. 172).

Das sechste und letzte Prinzip der CPA beinhaltet, dass auf der Grundlage der vollzogenen Analyse und im Austausch der Forschenden tentative Prozessmodelle zu bedeutsamen Therapieereignissen entwickelt werden sollen. Diese können 
durch thematische Auswertungen mehrerer Modelle auch in übergeordnete Modelle übergeführt werden (ebd., S. 173).

Tabelle 2.15 Framework for comprehensive process analysis (Quelle: Elliott 1989, S. 170)

\section{Framework}

\section{Expansion of implicit and explicit propositions in event}

(What is said «between the lines», including key propositions in event and derived by client)

\section{Contextual factors}

A. Background (relevant features of client and therapist which preceded and were brought to treatment, including client basic interpersonal conflicts, client style/ symptoms, client situation, therapist personal characteristics, therapist treatment principles)

B. Presession context (important events that have occurred since treatment began, in or out of treatment, including extratherapy events, previous sessions)

C. Session context (important sessions or features of the session in which the significant event occurs, including client and therapist tasks, alliance, relevant events)

D. Episode context (what has been happening in the current episode leading up to the event, including client and therapist tasks, relevant events, local cue)

\section{Event factors}

(Characteristics of the client and therapist's responses within the event that explain its significance)
A. Action (tasks and response modes within the event)
B. Content (what is being talked about that is so relevant to the impact)
C. Style/state (e.g., friendly, long, vivid)
D. Quality (extent to which client and therapist are working skillfully or well at the relevant therapeutic tasks)

\section{Impact of Client}

A. Process impact pathway (the sequence of observable responses by client and therapist during and immediately following the event)

B. Client experience pathway (the sequence of internal experienced responses by the client during and immediately following the event)

C. Delays impact of event (subsequent therapeutic impacts and changes in client apparently due to event)

D. Effectiveness of event (quantitative measures bearing on the effectiveness of the event, including immediate impact (within session), session outcome, delayed effectiveness (e.g. one-month follow-up), treatment outcome) 


\section{Vier Arbeitsschritte}

Den sechs Prinzipien weist Elliott vier Arbeitsschritte zu: erstens die Sammlung, zweitens die Analyse von Einzelereignissen (analysis of single events), drittens die Analyse der gesammelten Ereignisse (analysis of collections of significant events) und viertens die Erfassung und Überprüfung der Erwartungen und Annahmen der Beobachter (ebd.). Im ersten Arbeitsschritt werden sechs bis zehn als ähnlich wichtig eingestufte bedeutsame Therapieereignisse einer Klasse ausgewählt. Dazu werden Patientinnen oder Patienten in Interviews, Fragebögen oder «tape-assisted recall»-Verfahren gefragt, welche Momente ihrer Therapien sie als besonders hilfreich erlebt haben. Im zweiten Arbeitsschritt werden die identifizierten Ereignisse einschließlich des episodischen Kontexts transkribiert. Die Forschenden fertigen umfassende Sitzungsnotizen an, um wichtige Aussagen von Therapeut und Patientin festzuhalten und um beobachten zu können, was nach diesen bedeutsamen Ereignissen weiter geschieht. Die Bedeutung der Ereignisse soll herausgearbeitet werden («expansion of meanings in event»). Das heißt, es sollen Vorstellungen darüber entwickelt werden, was der Patient und die Therapeutin wirklich gesagt haben und welche Schlüsselbedeutungen («key meanings») für die Patienten oder Patientinnen darin erkennbar werden. Jetzt entwerfen die Forschenden je individuell einen «Ereignispfad» des bedeutsamen Ereignisses inklusive darauf bezogener Einflussfaktoren und Folgen für die Patienten und Patientinnen. In argumentativer Auseinandersetzung müssen sie sich auf eine Variante der Expansion und des Ereignispfades einigen ${ }^{9}$ (ebd., S. 177).

Sobald die Ereignispfade entwickelt sind, werden mittels Grounded Theory im dritten Arbeitsschritt (= «Analyse der Sammlung von Ereignissen») die ausgewerteten Fälle zueinander in Beziehung gesetzt und gemeinsame relevante Themen analysiert. Auf diese Weise sollen fallübergreifende Modelle entwickelt werden, die Auskunft darüber geben, wie bedeutsame Therapieereignisse entstehen und sich entfalten. Als Ergebnis dieser Analysen spricht Elliott von «allgemeinen Modellen» wie etwa dem «General Model of Insight Events» (Elliott et al. 1994, S. 460). Als Variante davon beschreibt Elliott «Mikromodelle», in denen im Unterschied zu den allgemeinen Modellen kurze Sequenzen der Ereignispfade sehr präzise dargestellt werden. Bezugsgröße sind hier einzelne Sprechakte der

9 Gibt es keine Einheit unter den Forschenden, muss das «Mehrheitsprinzip» angewendet werden. Ein Test zur Unterscheidung, ob ein Element in den Ereignispfad aufgenommen werden soll oder nicht, besteht zudem darin, das Element auf drei Kriterien hin zu prüfen: 1) Ist es in Bezug auf das Ereignis tatsächlich gegenwärtig? 2) Ist das Element nicht mit anderen Elementen redundant? Und 3): Ist das Element in Bezug auf das Zustandekommen oder die Erklärung des Ereignisses oder seiner Folgen wichtig? (Vgl. dazu Elliott 1989, S. 177). 
Therapeuten sowie deren Folgen für die Patienten oder Patientinnen, die während der bedeutsamen Ereignisse konkret beobachtet werden konnten (Elliott 1989, S. 180-182). Im vierten und letzten Arbeitsschritt werden die vor der Auswahl der bedeutsamen Ereignisse explizierten Annahmen der Forschenden hinsichtlich relevanter Einflussfaktoren und Ereignisfolgen mit den tatsächlichen Ergebnissen verglichen (Elliott 1989, S. 183).

\section{Methodologische Grundlagen}

Elliott ordnet die CPA der qualitativen Sozialforschung und Methodologie zu. Dazu zählt er besonders Methoden zur Erhebung phänomenologischer Daten (Erlebensperspektiven der Patienten/Therapeutinnen) sowie die interpretative Analyse und Auswertung in Anlehnung an das CPA-Framework.

\subsection{3 «Assimilation Analysis»}

\section{Ziel}

In der «Assimilation Analysis» werden psychologische Veränderungsprozesse von Patientinnen und Patienten untersucht. Die Leitfrage ist, wie Patienten oder Patientinnen «problematische Erfahrungen» in Psychotherapien in bestehende Schemata integrieren (Stiles \& Angus 2001, S. 112), ${ }^{10}$ um so deren negative Wirkungen aufzulösen. Das übergeordnete Forschungsziel der «Assimilation Analysis» liegt darin, das bereits entwickelte «Assimilation Model» als allgemeines psychologisches Veränderungsmodell fortwährend weiter zu differenzieren.

\section{Ursprung, Grundbegriffe und Grundannahmen}

Die «Assimilation Analysis» als Forschungsansatz und das daraus entwickelte «Assimilation Model» entstanden als Folge missratener Versuche, psychotherapeutische Prozesse mit finalen Therapieergebnissen auf der Grundlage linearer statistischer Modelle miteinander in Beziehung zu setzen (Stiles 2006, S. 389).

10 In einem neueren Beitrag (Stiles \& Angus 2001, S. 112) beschreibt Stiles, dass es mittlerweile unterschiedliche Formulierungen des «Assimilation Model» gibt. Neben der ursprünglichen Schemaversion (Stiles et al. 1990), die auf die kognitive Entwicklungstheorie nach Piaget (1970) zurückgeht, gibt es auch die Variante der Assimilation problematisch erscheinender innerer Stimmen in ein dominantes Stimmenensemble (Honos-Webb \& Stiles 1998) sowie die auf die Kognitionswissenschaften zurückgehende Version der Verlinkung unvereinbar erscheinender Erinnerungen (Williams, Stiles \& Shapiro 1999). 
Begrifflich wurden «problematische Erfahrungen» als «a memory, wish, feeling, idea or attitude that is threatening or painful to the client» (Stiles \& Angus 2001, S. 113) definiert, wobei Stiles gemeinsam mit Angus den Schemabegriff in Anlehnung an Piaget definiert:

«The term schema is used in a very broad sense; it might be a frame of reference, way of living, narrative, metaphor, or theme. As in Piaget's theory, assimilating a problematic experience requires accommodations in existing schemata or sometimes the development of new schemata within the therapist-client relationship.» (Stiles \& Angus 2001, S. 113)

Das «Assimilation Model»(vgl. Abbildung 2.6) ist gemäß Stiles integrativ, da damit über verschiedene Therapieprobleme und -schulen hinweg in Therapien auftretende Veränderungsabläufe beschrieben werden. Das Modell umfasst folgende Stufen der Bewältigung problematischer Erfahrungen: 1) Abwehr, 2) ungewollte Gedanken, 3) vages Bewusstsein oder Auftauchen des Problems, 4) Problemäußerungen oder -klärung, 5) Verständnis oder Einsicht, 6) Durcharbeiten oder Anwendung, 7) Problemlösung und 8) Meisterung/Bewältigung [eigene Übersetzung] (ebd., S. 114). Von Abwehr wird zum Beispiel dann gesprochen, wenn es Belege dafür gibt, dass der Patient oder die Patientin aktiv versucht, emotional unangenehme Themen zu meiden - zum Beispiel, indem er oder sie von einem von der Therapeutin angesprochenen Thema zu einem anderen wechselt.

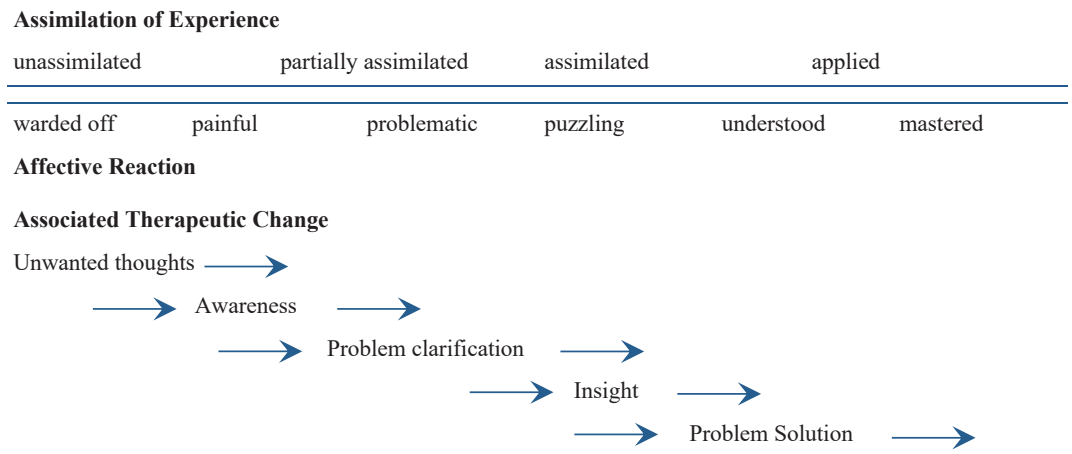

Abbildung 2.6 Kontinuum der Assimilation problematischer Erfahrungen, affektiver Reaktionen und damit verbundener Wirkungen (Quelle: Stiles et al. 1990, S. 413) 


\title{
Analyseschritte
}

Der Forschungsansatz der «Assimilation Analysis» beruht auf vier Arbeitsschritten. Im ersten Schritt soll der Forscher sich mit dem Datenmaterial vertraut machen und interessante Passagen identifizieren. Dazu werden Transkripte mehrfach gelesen und Aufzeichnungen mehrfach angehört (ebd., S. 116). Im zweiten Analyseschritt werden die behandelten problematischen Erfahrungen identifiziert. Eine Möglichkeit dazu besteht darin, nach «Momenten der Einsicht» oder nach solchen «des neuen Verständnisses» zu suchen. Mit diesem Hinweis ist die Annahme verbunden, dass in solchen Momenten Themen enthalten sind, die im Verlauf der Therapie produktiv bearbeitet wurden und weiter werden. Der dritte Analyseschritt besteht dann darin, zu den problematischen Erfahrungen weitere Gesprächspassagen zu identifizieren. Stiles verweist darauf, dass mit der zunehmenden Assimilation problematischer Erfahrungen sich die Wortwahl sowohl der Patienten als auch der Therapeutinnen oft verändert. Dies sollen die Forschenden bei der Auswahl der Passagen berücksichtigen. Im vierten und letzten Arbeitsschritt wird der Assimilationsprozess beschrieben. Ziel dabei ist, aufgrund der beobachtbaren Veränderung der problematischen Erfahrung, die theoretische Beschreibung des «Assimilation Model» zu bestätigen, zu korrigieren oder zu erweitern.

\section{Methodologische und erkenntnistheoretische Grundlagen}

Methodologisch vertritt Stiles einen qualitativen, einzelfallstudienbasierten und theoriebildenden Forschungsansatz (Stiles 2007). Dieser orientiert sich wie die «Task Analysis» an der methodologischen Position der Abduktion nach Peirce (Brinegar et al. 2006, S. 165). Dazu folgendes Zitat:

\begin{abstract}
"Rather, theory is a flexible (permeable) account that was constructed as a way of understanding previous observations and now must be elaborated, extended, qualified, and modified to encompass the observations at hand. This constructive process was termed <abduction〉 by the philosopher Charles Peirce (1965; Rennie 2001). If the observations do not fit, then the theory has to change so that it incorporates the new observations while still making sense of the past observations upon which it was built. In this way, qualitative case study research can be cumulative (Stiles 2005a). Theory-building research thus imposes a responsibility on investigators to be informed about the theory and previous research as well as to remain permeable to observations that contradict or go beyond the theory.»
\end{abstract}

Die methodologische Position von Stiles wird erkennbar in der Beschreibung des Verhältnisses von qualitativen Einzelfallstudien, auf der die «Assimilation Analysis» gründet, und dem «Assimilation Model», dem übergeordneten Forschungs- 
gegenstand dieser Analysen. Denn eine durchgeführte «Assimilation Analysis» führt idealerweise dazu, den untersuchten Fall und das «Assimilation Model» besser zu verstehen. Da jeder Fall einzigartig ist, ist es gemäß Stiles möglich, das Modell durch weiterführende Beschreibungen der einzelnen Phasen zu präzisieren. Darüber hinaus kann mit jedem neuen Fall geprüft werden, inwiefern das «Assimilation Model» gar abgelehnt werden muss. In Bezug auf diesen Schritt hält Stiles relativierend fest, dass vorhergehende Untersuchungen als konservierende Kräfte fungieren und Anpassungen des Modells immer mit daraus generierten Erkenntnissen übereinstimmen müssen (Stiles \& Angus 2001, S. 117).

Den Prozess der Elaboration und der Korrektur versteht Stiles als Antwort auf die Frage, wie qualitative Forschung aggregiert werden kann. So geht er davon aus, dass Beobachtungen durch eine Umwandlung des Verständnisses der Forschenden aggregiert werden und nicht durch einen Wissenszuwachs. Jede neue Beobachtung verändert das vorherige Verständnis des Wissenschaftlers oder der Wissenschaftlerin. Dieses Verständnis muss aber immer auch ältere Beobachtungen einschließen. Entsprechend verändert sich das «Assimilation Model» fortwährend, wenngleich der Name des Modells bleibt, entsprechend der Idee des hermeneutischen Zirkels (ebd.).

Seine erkenntnistheoretische und ontologische Position bezeichnet Stiles als «experiental correspondence theory of truth». Er umschreibt sie zusammen mit Brinegar wie folgt (Brinegar et al. 2006, S. 165):

«Our use of theory-building research assumes a constructivist epistemology within a realist ontology, elsewhere described as the experiential correspondence theory of truth (Stiles 1981; Stiles 2005b). This suggests that a statement is true to the extent that the experience of hearing it corresponds to the experience of observing the events it describes. Observations and descriptions of observations, insofar as they represent human experience, are approximate, fallible, and variable across time and people. Nevertheless, within the limits of human communication, changes to theory, such as those developed in this study, must fit coherently into the array of assumptions, terms, mechanisms, and tenets that have evolved from previous research.»

\subsubsection{Vergleichende Zusammenfassung der «Significant Event Design»-orientierten Psychotherapie-Forschungs- ansätze}

Den drei am «Significant Event Design» orientierten Psychotherapie-Forschungsansätzen ist gemein, dass sie bedeutsame Ereignisse auf der Grundlage faktischer Therapieverläufe untersuchen. Als Unterscheidungsmerkmal lässt sich festhalten, 
dass sich bedeutsame Ereignisse in der «Task Analysis» auf spezifische Therapieprobleme beziehen. Diese werden als Therapieaufgaben aufgefasst, so etwa das Überwinden unverarbeiteter Gefühle oder der Umgang mit Beziehungsbrüchen. Dabei wird untersucht, welche Teilaufgaben bearbeitet werden müssen, sodass die Patientin oder der Patient die Therapieaufgabe erfolgreich bearbeiten respektive das Problem überwinden kann. Als Forschungsergebnisse werden in der «Task Analysis» - in der Auseinandersetzung mit Erfahrungs- und Theoriewissen der Forschenden sowie der Analyse faktischer Sitzungen - theoretisch-empirische Modelle der erfolgreichen Bearbeitung ausgewählter Therapieaufgaben gebildet. Methodologisch orientiert sich die «Task Analysis» am Modell der Abduktion, dessen Leitgedanke darin besteht, theoretische Vorannahmen in der Auseinandersetzung mit empirischen Daten zu differenzieren. Erkenntnistheoretisch verortet sich die «Task Analysis» im «dialectical constructivism». Damit gemeint ist, dass die Wirklichkeit nie vollständig objektiv beobachtet und festgehalten werden kann, aber dennoch Begrenzungen aufweist. Im Rahmen dieser Begrenzungen wird unser Wissen konstruiert.

Ein wichtiger Unterschied der «Comprehensive Process Analysis» gegenüber der «Task Analysis» besteht darin, dass verschiedene Klassen bedeutsamer Ereignisse analysiert werden, so beispielsweise «Momente der Einsicht». Besonders am Ansatz ist weiter, dass die Wichtigkeit von Ereignissen in einer Therapie als stark variierend bewertet wird. Bedeutsame Ereignisse werden definiert als «turning points», in denen Patientinnen und Patienten positive psychische Wirkungen erfahren. Ein Beispiel hierfür ist - bezogen etwa auf das Bedürfnis, sich selbst besser verstehen zu wollen - ein «Moment der Einsicht», der durch eine Therapeuteninterpretation mitgestaltet wird. Die Forschungsergebnisse, die in der «Comprehensive Process Analysis» im Unterschied zur «Task Analysis» entwickelt werden, sind durch die Forschenden - in der Auseinandersetzung mit Theoriewissen und der Analyse faktischer Therapieverläufe - gebildete, unterschiedlich abstrakte Modelle der untersuchten Ereignisklassen. Methodologisch wird der Ansatz, im Unterschied zur «Task Analysis» und zur «Assimilation Analysis», der induktiven qualitativen Sozialforschung zugerechnet.

Kernmerkmal der «Assimilation Analysis» ist, dass das «Assimilation Model» als allgemeine Veränderungstheorie für Patienten und Patientinnen fortwährend weiterentwickelt wird. Bedeutsame Ereignisse beziehen sich hier auf die unterschiedlichen Phasen (z.B. Problemäußerung, Einsicht), die ein Patient oder eine Patientin durchlaufen muss, um «problematische Erfahrungen» in die bestehenden Schemata zu integrieren, womit deren negative Wirkungen aufgelöst werden können. Analog zur «Task Analysis» und anders als bei der «Comprehensive Process Analysis» werden, vermittelt über das «Assimilation Model», einzelne Therapie- 
ereignisse mit den finalen Ergebnissen einer Therapie in Verbindung gesetzt. Zudem wird der Ansatz wie die «Task Analysis» und anders als die «Comprehensive Process Analysis» dem methodologischen Modell der Abduktion zugeordnet. Im Unterschied zur «Task Analysis» beruht die «Assimilation Analysis» dafür auf Einzelfallstudien. Der erkenntnistheoretische Rahmen der «Assimilation Analysis» ist die «experiential correspondence theory of truth», in der Aussagen dann als wahr bewertet werden, wenn die Erfahrung, diese zu hören (= zu lesen), mit der Beobachtung der Ereignisse übereinstimmt.

Im Folgenden werden die wichtigsten Erkenntnisse dieser drei psychotherapiebezogenen Forschungsansätze tabellarisch abgebildet (vgl. Tabelle 2.16). 


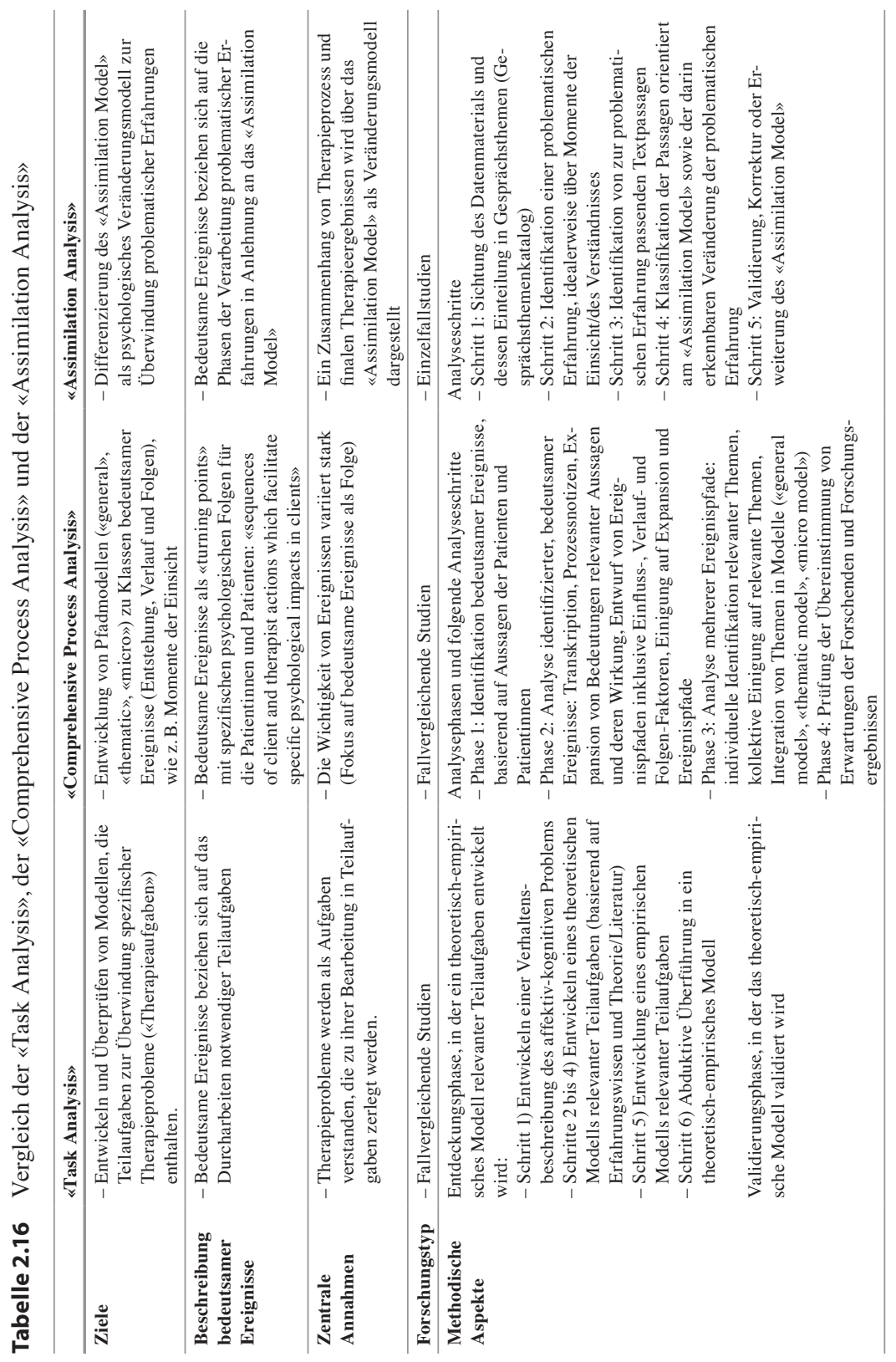




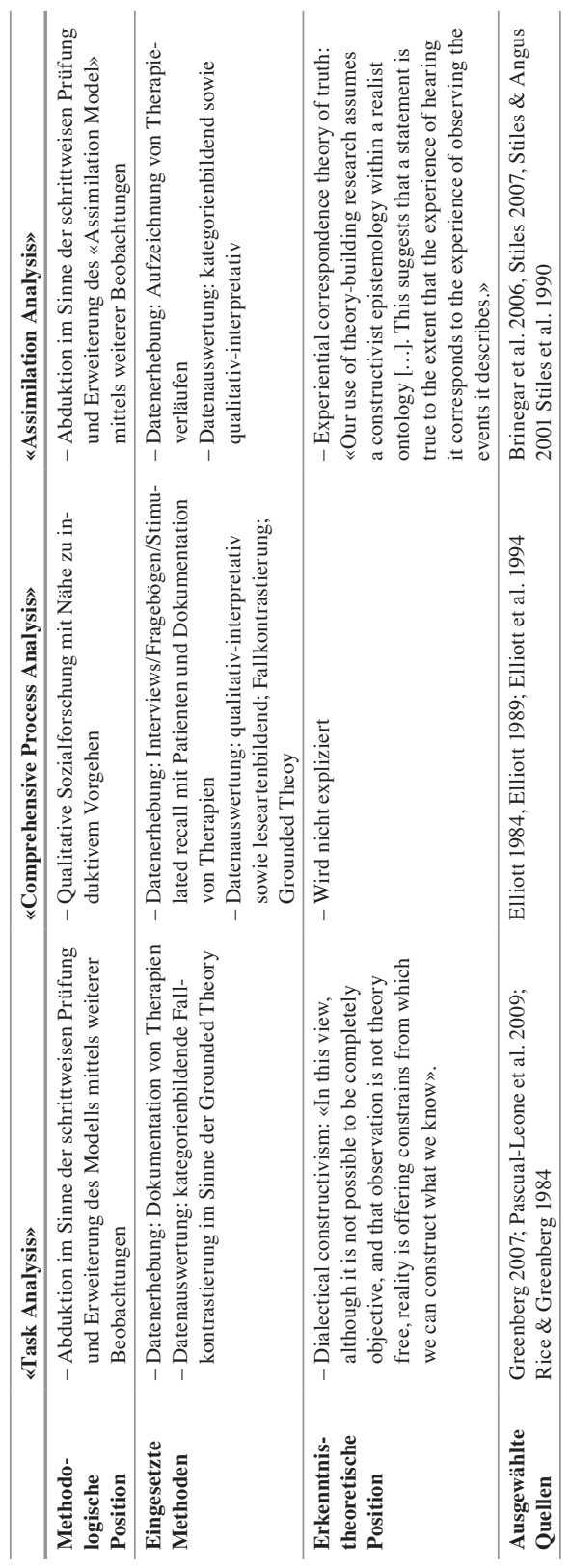




\subsubsection{Erste methodologische, theoretisch-inhaltliche und methodische Bestimmung der Untersuchung zu «bedeutsamen Momenten» im Coaching}

Wie erwähnt, wurden die drei psychotherapiebezogenen Forschungsansätze deshalb vorgestellt, weil es bis dato keine entsprechenden Untersuchungen zu «bedeutsamen Ereignissen» im Coaching gibt und das Ziel der vorliegenden Studie darin besteht, in diese Richtung einen ersten Schritt zu gehen. So liegt die Absicht der Präsentation dieser Forschungszugänge als Ausgangspunkt und Inspirationsgrundlage darin, erste methodologische, inhaltlich-theoretische sowie methodische Aspekte für die vorliegende Studie zu bestimmen, die dann in Kapitel 3 präzisiert werden.

Wie noch detaillierter ausgeführt wird, fällt, bezogen auf den methodologischen Rahmen der vorliegenden Studie - analog zur «Task Analysis» und zur «Assimilation Analysis»-die Entscheidung auf das Modell der Abduktion. Für die inhaltliche Bestimmung von «bedeutsamen Momenten» im Coaching werden Anleihen bei der «Task Analysis», der «Comprehensive Process Analysis» und bei den linguistischen Rekonstruktionen von Graf gemacht. Bezogen auf methodische Aspekte, wird in Anlehnung an die «Comprehensive Process Analysis» die Idee einer interpretativen Vorgehensweise zur Rekonstruktion des Verlaufs und der Dynamik bedeutsamer Ereignisse aufgegriffen. Darüber hinaus verfolgen wir analog zur «Task Analysis» und zur «Assimilation Analysis» die Absicht, die «bedeutsamen Ereignisse» zu den finalen Coaching-Ergebnissen in Beziehung zu setzen.

\section{Ein abduktiver Forschungsrahmen}

Mit dem Ziel, ein in sich schlüssiges methodisches Verfahren zu entwickeln, mittels dessen «bedeutsame Momente» im Coaching im Rahmen gesamter Coaching-Verläufe identifiziert und analysiert werden können, wurde in einem ersten Schritt ein für die Studie passender methodologischer Rahmen bestimmt. Aufgrund der vorhandenen theoretischen Vorstellungen $\mathrm{zu}$ «bedeutsamen Momenten» im Coaching, die gleich näher vorgestellt werden, und des Anspruchs, diese Vorstellungen durch die Analyse empirischer Daten zu konkretisieren, lag der Entscheid auf der Hand, für die vorliegende Untersuchung, analog zur «Task Analysis» (vgl. Kapitel 2.4.1) und zur «Assimilation Analysis» (vgl. Kapitel 2.4.3), ein abduktives Vorgehen zu wählen.

Die Idee dieser methodologischen Position besteht darin, theoretische Vorstellungen zum Forschungsgegenstand - im vorliegenden Fall die «bedeutsamen Momente» im Coaching - als forschungsleitendes Konzept einzuführen. Im Unterschied zu deduktiv-hypothesenprüfenden Vorgehensweisen fungieren diese Vor- 
stellungen nicht als zu überprüfende These, sondern als sensibilisierendes Konzept. In der Auseinandersetzung mit den empirischen Daten soll dieses Konzept in einem kreativen und regelgeleiteten Prozess in ein definitives, zumindest aber in ein differenzierteres Konzept übergeführt werden (Kelle \& Kluge 2010, S. 29).

\section{«Bedeutsame Momente» im Coaching als sensibilisierendes Konzept}

Als sensibilisierendes Konzept der geplanten Untersuchung entstand in dieser Arbeit also ein erstes Verständnis von «bedeutsamen Momenten» im Coaching in Anlehnung an das Verständnis bedeutsamer Therapieereignisse in der «Task Analysis» und der «Comprehensive Process Analysis» sowie unter Rückgriff auf die von Graf vorgestellten strukturellen, thematischen und interaktiven Merkmale von Coaching-Prozessen (vgl. Kapitel 2.3.2, S. 70 ff.). Im Folgenden wird dieses Verständnis und dessen Herleitung zusammenfassend dargestellt.

Zur theoretischen Bestimmung «bedeutsamer Momente» im Coaching erwies es sich als nützlich, zunächst einmal Coaching-Prozesse als übergeordnete Kategorie zu bestimmen. Dies erfolgte in Bezug auf das in der «Task Analysis» enthaltene Prozessverständnis (vgl. Kapitel 2.4.1), dessen zentrale Annahme lautet, dass Patientinnen und Patienten zur Bewältigung ihrer therapeutischen Probleme (gerahmt als therapeutische Aufgaben) dazu passende Teilaufgaben lösen müssen. Die Bestimmung von Coaching-Prozessen bezog sich weiter auf zwei Annahmen der dieser Arbeit zugrunde gelegten Coaching-Definition (vgl. Kapitel 1.4.2): erstens, dass Coachees in einem Coaching in aller Regel berufliche Ziele verfolgen und zur Erreichung dieser, zweitens, innere Veränderungsprozesse im Sinne der Ausbildung funktionaler Deutungs- und Handlungsmuster vollziehen. Zudem gehen wir analog zur Position von Eva-Maria Graf davon aus, dass Coach und Coachee das Anliegen eines Coachee durch einen (kommunikativen) Ko-Konstruktionsprozess bearbeiten.

Folgt man nun der Annahme, dass berufliche Leistungs- und Handlungsziele analog zu psychischen Problemen (vgl. «Task Analysis») als Aufgaben verstanden werden, resultiert daraus, dass Coaching-Prozesse kommunikative Ko-Konstruktionsprozesse der Bewältigung von Teilaufgaben sind, die zur Erreichung identifizierter Coaching-Ziele führen und beim Coachee auf entsprechenden Veränderungsprozessen im Sinne der Ausbildung funktionaler Deutungs- und Handlungsmuster fußen.

An dieser Stelle erfolgt nun eine Übertragung dieser Ausführungen auf das Konzept der «bedeutsamen Momente» im Coaching. Orientiert an der Idee von «turning points» (vgl. «Comprehensive Process Analysis»), Momenten also, in denen sich für die Coachees etwas zum Positiven wendet, können «bedeutsame Momente» im Coaching zunächst als produktive Verschränkungen von Coachee- 
und Coach-Interventionen definiert werden. In diesen Momenten erleben die Coachees, analog zur Beschreibung in der «Comprehensive Process Analysis», psychologische Wirkungen, die sich in Form mentaler Veränderungsprozesse zeigen. Mit Bezug auf das eben beschriebene Verständnis von Coaching-Prozessen bedeutet dies dreierlei: Erstens fungieren die mentalen Veränderungsprozesse als Treiber der Ausbildung funktionaler Deutungsmuster und werden zweitens entsprechend den Ausführungen von Graf (vgl. Kapitel 2.3.2, S. 70 ff.) in der Interaktion von Coachee und Coach kommunikativ ko-konstruiert. Drittens beziehen sich die mentalen Veränderungsprozesse auf zu bearbeitende Teilaufgaben, die in einem produktiven Verhältnis zum Coaching-Anliegen und zu den damit verbundenen Coaching-Zielen stehen. Dabei gilt es zu präzisieren, dass bereits die Klärung von Coaching-Zielen einen mentalen Veränderungsprozess bedingen kann, da viele Coachees zu Beginn eines Coachings noch nicht in der Lage sind, ihre Ziele klar zu artikulieren (vgl. dazu auch Schreyögg 2012).

Entsprechend diesen Bestimmungen, wird in dieser Arbeit dann von «bedeutsamen Momenten» im Coaching gesprochen, wenn folgende drei Kriterien erfüllt sind:

- Erstens müssen Coachees während dieser Momente mentale Veränderungsprozesse vollziehen.

- Zweitens sind diese mentalen Veränderungsprozesse Ausdruck der erfolgreichen Bearbeitung von Teilaufgaben, die in einem produktiven Verhältnis zum Coaching-Anliegen und zum damit verbundenen Coaching-Ziel stehen.

- Drittens müssen sie als Ko-Konstruktionen in der Interaktion von Coach und Coachee entstehen.

\section{Erste methodische Bestimmungsmerkmale}

Im Anschluss an diese erste methodologische und inhaltlich-theoretische Bestimmung besteht die zentrale methodische Frage darin, wie in Bezug auf das vorliegende Coaching-Fallmaterial Teilaufgaben für die vertiefende Analyse der darin potenziell enthaltenen «bedeutsamen Momente» identifiziert werden können, die in einem produktiven Verhältnis zum Coaching-Anliegen und zum damit verbundenen Coaching-Ziel stehen. Die präzise Beschreibung des methodischen Vorgehens erfolgt in Kapitel 3. Als Vorwegnahme und erste Orientierung sei an dieser Stelle darauf hingewiesen, dass sich die Identifikation solcher Teilaufgaben auf die Rekonstruktion des Entwicklungsprozesses des Coachee über den gesamten Coaching-Fall bezieht.

Vorweggenommen wird weiter, dass die Analyse «bedeutsamer Momente» im Coaching selbst unter der Berücksichtigung des «sinnerschließenden Inter- 
pretationssystems» von Geißler erfolgen wird. Das bedeutet, dass zur Rekonstruktion der spezifischen Dynamik dieser Momente relevante Kommunikationsentscheide von Coach und Kunde oder Kundin interpretiert werden.

Im nun folgenden Kapitel wird auf der Grundlage dieser ersten Bestimmungsmerkmale das Forschungsdesign für die vorliegende Studie präzisiert.

Open Access Dieses Kapitel wird unter der Creative Commons Namensnennung 4.0 International Lizenz (http://creativecommons.org/licenses/by/4.0/deed.de) veröffentlicht, welche die Nutzung, Vervielfältigung, Bearbeitung, Verbreitung und Wiedergabe in jeglichem Medium und Format erlaubt, sofern Sie den/die ursprünglichen Autor(en) und die Quelle ordnungsgemäß nennen, einen Link zur Creative Commons Lizenz beifügen und angeben, ob Änderungen vorgenommen wurden.

Die in diesem Kapitel enthaltenen Bilder und sonstiges Drittmaterial unterliegen ebenfalls der genannten Creative Commons Lizenz, sofern sich aus der Abbildungslegende nichts anderes ergibt. Sofern das betreffende Material nicht unter der genannten Creative Commons Lizenz steht und die betreffende Handlung nicht nach gesetzlichen Vorschriften erlaubt ist, ist für die oben aufgeführten Weiterverwendungen des Materials die Einwilligung des jeweiligen Rechteinhabers einzuholen.

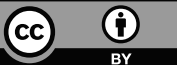

